\title{
Semantic influences on episodic memory distortions
}

\author{
Alexa Tompary ${ }^{1^{*}}$ and Sharon L. Thompson-Schill ${ }^{1}$ \\ 1Department of Psychology, University of Pennsylvania, Philadelphia, PA 19104
}

"Corresponding author:

Alexa Tompary

atompary@sas.upenn.edu

72 pages, 6 figures, 1 table

Word count, abstract: 245

Word count, article body: 15,593 
2 Prior knowledge can facilitate or distort new episodic memories, depending on their

3 alignment. However, it remains unclear how the structure of semantic memory explains

4 systematic variation in how new memories are formed and retrieved. We aimed to

5 quantify distortions in memory by examining how category membership and typicality

6 bias new memories. Across four experiments, participants encoded and retrieved

7 image-location associations. Most members of a category (e.g. birds) were located near

8 each other, such that participants could learn locations of categories as they encoded

9 specific image locations. Critically, some typical and atypical category members were in

10 random locations. We decomposed location memory into two measures: error, a

11 measure of episodic specificity, and bias towards other category members, a measure

12 of the influence of prior knowledge. First, we found that location memory was more

13 accurate for images that were spatially consistent with their category membership.

14 Second, when images were spatially inconsistent, retrieval of typical category members

15 was more biased towards other category members relative to atypical ones. These

16 effects replicated across three experiments, disappeared when images were not

17 arranged by category, and were stronger than effects observed with images arranged

18 by visual similarity rather than category membership. Our observations provide

19 compelling evidence that memory is a reconstruction of multiple sources of information,

20 integrating memory for specific events with relevant semantic knowledge. Furthermore,

21 systematic differences in the magnitude of this integration suggest that the organization

22 of semantic memory can govern the extent of distortion in new episodic memories.

23 Key words: Episodic memory, semantic memory, memory reconstruction, distortion 
25 Episodic and semantic memory are commonly studied as distinct cognitive 26 phenomena, the former defined as memory for 'personal experiences and their temporal 27 relations' and the latter as memory for the 'meaning of words, concepts, and 28 classification of concepts' (Tulving, 1972). While this distinction has led to important 29 characterizations of both memory systems (e.g., A. M. Collins \& Loftus, 1975; 30 McClelland, McNaughton, \& O'Reilly, 1995) and fruitful investigations of the interactions 31 between them (e.g., McKoon \& Ratcliff, 1979; Anderson \& Ross, 1980; Simons, 32 Graham, \& Hodges, 2002), it also oversimplifies the complexity of memories, which 33 comprise both episodic and semantic elements. In other words, it neglects the critical 34 notion that new experiences are made up of re-combinations of objects, places, and 35 people for which we already have rich semantic knowledge. We aimed to disentangle 36 the separable contributions of semantic and episodic elements to new memory

37 formation and/or retrieval by quantifying how semantic knowledge distorts new episodic 38 learning.

39 The influence of prior knowledge on new episodic memories is widely 40 documented (Bartlett, 1932; Ghosh \& Gilboa, 2014). On the one hand, prior knowledge 41 can enhance new memories (Bransford \& Johnson, 1972; Craik \& Tulving, 1975).

42 Similarly, the presence of prior knowledge accelerates the integration of novel words 43 into existing memory networks (Coutanche \& Thompson-Schill, 2014). At the same 44 time, new encoding can also be biased by prior knowledge, resulting in false memories 45 or confabulation (Koutstaal \& Schacter, 1997; Webb, Turney, \& Dennis, 2016) or 46 distorted details of true memories (Bartlett, 1920; Loftus \& Palmer, 1974). Taken 
47 together, these findings suggest that prior knowledge can help or hinder memory for 48 new events.

49 What dictates whether prior knowledge is helpful or harmful for new memories? One 50 promising approach to answer this question is to consider retrieval as a construction of

51 different sources of information. Such an account has been useful in explaining errors in

52 a wide variety of cognitive phenomena, including but not limited to object perception, 53 color constancy, and visual short term memory (e.g. Knill \& Richards, 1996; Brainard et

54 al., 2006; Heussen, Poirier, Hampton, \& Aldrovandi, 2011). According to this view, 55 retrieval is not a veridical recapitulation of past events, but instead an imperfect 56 recombination of event-specific details and other knowledge (Addis, Pan, Vu, Laiser, \& 57 Schacter, 2009). Because episodic memories are often noisy and incomplete, 58 successful remembering is thought to combine these partial representations with 59 knowledge from prior experiences (Huttenlocher, Hedges, \& Vevea, 2000). Integrating 60 prior knowledge with episodic memories can thus be thought of as a way to improve the 61 'signal' of a memory, resulting in more accurate retrieval. Yet, it also introduces 62 systematic errors if there are discrepancies between a new memory and prior 63 knowledge. In long-term memory, exposure to semantically related words (e.g., sour, 64 candy, sugar) often produces a false memory for a non-studied word (sweet; Roediger 65 \& McDermott, 1995). Such errors are also captured with continuous measures of bias; 66 for example, memory for the spatial locations of dots is often biased towards the 67 horizontal and vertical axes of the stimulus display, suggesting that participants group 68 memory for continuous locations into discrete chunks (Huttenlocher, Hedges, \& 69 Duncan, 1991), memory for the color of shapes is biased towards canonical hues 
70 (Persaud \& Hemmer, 2016), and estimates of the size of fruits and vegetables are

71 biased by both their superordinate and subordinate mean sizes (P. Hemmer \& Steyvers,

72 2009). Taken together, these findings provide compelling evidence that long-term

73 memory is a reconstruction of prior knowledge and new experiences and accounts for

74 variation in how prior knowledge interacts with new memories.

75 However, one weakness of this work is that the operationalization of prior 76 knowledge often ignores its rich, dimensional structure (A. Collins \& Quillian, 1969). In

77 such a structure, concepts vary in the similarity of their features, giving rise to 78 categories. Typical category members are defined as items that share the greatest

79 number of features with other members, and thus are the best examples of that 80 category (E. Rosch, Mervis, Gray, Johnson, \& Boyes-Braem, 1976). As a result, typical 81 category members are thought to be more strongly associated with category neighbors,

82 relative to atypical ones. The consequences of these strong associations are well 83 documented: Typical category members are more quickly categorized, more efficiently 84 recognized, and less resistant to disruption by brain damage (Patterson, 2007). 85 Furthermore, the features of typical category members are more often attributed to 86 category neighbors (Rips, 1975; Osherson, Smith, Wilkie, López, \& Shafir, 1990). As an 87 example, if a typical category member, rather than an atypical category member, is 88 accompanied by a shock, participants are more likely to anticipate shocks with other 89 category members (Dunsmoor \& Murphy, 2014). Examining how new memories are 90 formed in the context of this structure may lead to a better understanding of the 91 reconstruction of episodic and semantic information when retrieving new memories. 
92 We aimed to quantify distortions in episodic memories due to prior knowledge by 93 examining how differences in category typicality bias new episodic memories. In a

94 series of behavioral experiments, participants encoded and retrieved image-location 95 associations on a 2D grid (Figure 1A). The locations associated with the images were 96 determined by semantic relatedness ratings, such that most members of the same 97 category (e.g. birds) were located near each other, but some typical and atypical 98 category members were in random locations (Figure 1B-D). This configuration allows 99 participants to learn that images from a certain category tend to be located in a certain 100 area as they encode the locations of specific images. This general knowledge could 101 only be learned and used if participants integrated learning of new image-specific 102 locations with their prior knowledge of animal and object categories. We used a 103 continuous retrieval measure to disentangle biases driven by semantic knowledge from 104 errors due to forgetting (Figure 1E). Error and bias could vary independently, such that 105 memory for an image could be biased towards or away from category neighbors at the 106 same level of accuracy. We used these measures to quantify the influence of 107 knowledge about a category's general location when retrieving the locations of each 108 image.

109 Under a reconstruction account, we predicted that the use of this category 110 knowledge would give rise to two distinct effects: (1) retrieval of images located near 111 members of the same category would be more accurate relative to those in random 112 locations, and (2) for images located in random locations, retrieval of typical category 113 members would be more biased in the direction of category neighbors relative to 114 atypical ones. Such a bias would reflect stronger associations between typical category 
115 members and their category neighbors. Finally, although we expected that category

116 typicality would specifically influence bias in memory, we also explored potential

117 differences in their accuracy.

118 We tested these predictions across four behavioral experiments (summarized in

119 Table 1). In Experiment 1, we developed a novel, data-driven procedure to determine

120 category membership and typicality for a set of animals and objects. In Experiment 2,

121 we designed a conceptual replication using a different procedure for defining category

122 typicality with validated and pre-determined categories. As a preview, both experiments

123 provided evidence in favor of the two predictions outlined above, suggesting that

124 participants relied on a mixture of event-specific details and category knowledge to

125 support retrieval and that the organization of this category knowledge explained

126 differences in distortion of new episodic memories. In Experiments 3 and 4, we

127 examined possible explanations for the observed differences in bias by category

128 typicality. In Experiment 3, we asked whether the utility of category knowledge during

129 encoding differentially influenced how typical and atypical category members

130 processed, by assessing memory for their perceptual details. In Experiment 4, we

131 developed a set of stimuli that could be organized by a non-semantic property (i.e.,

132 arbitrary image color) to examine whether the observed biases in memory were driven

133 by the visual similarity of the images rather than their conceptual structure. 
A

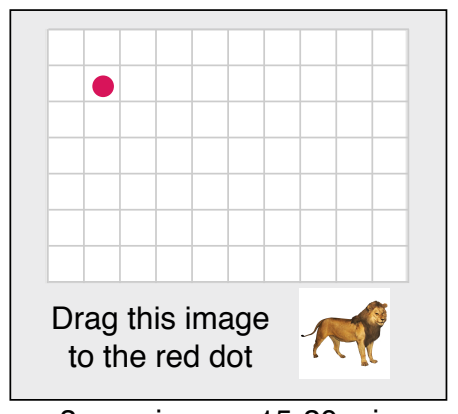

3x per image: $15-20 \mathrm{~min}$

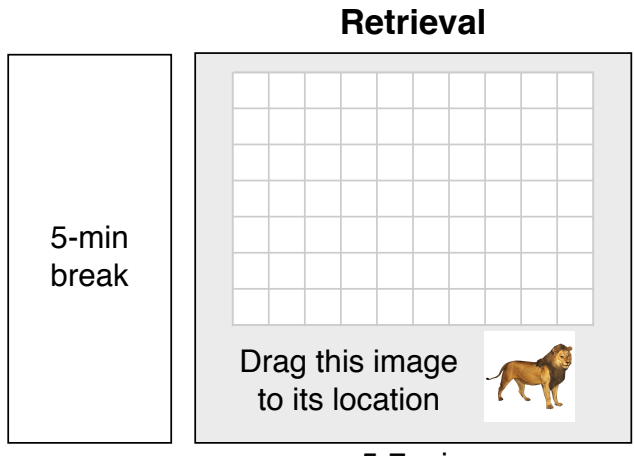

5-7 min
B

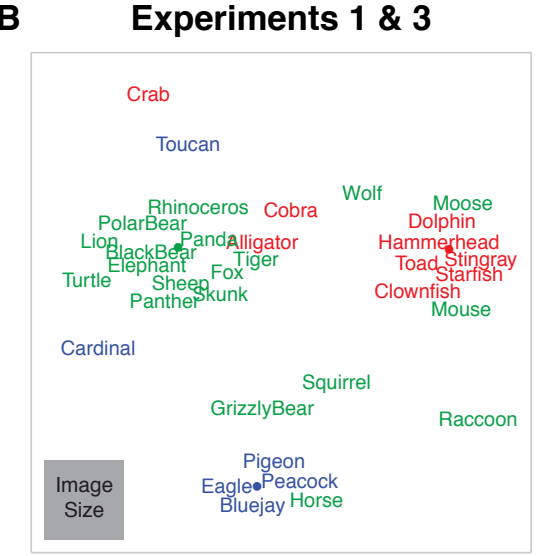

C

$$
\begin{aligned}
& \text { Manatee Flamingo Swordfish Shark Mussel } \\
& \text { Swallow Eagle Caterpillar Killer Whale } \\
& \text { Ant Duck Chicken Seal Starfish } \\
& \text { Goose Peacock Slug Horse } \\
& \text { Rooster Cogkroach Clownfish Rhinøceros Lobster } \\
& \text { Swan Pelican Sea Turtle Cardinal } \\
& \text { Crab HippopotamusHeron Sea Lion Shrimp } \\
& \text { Seagull Cow Penguin Squid Hammer } \\
& \text { Parrot Owl Stingray Head Shark } \\
& \text { Ostrich Butterfly Dragonfly Lion Scorpion } \\
& \text { Wasp GrasshopperToucan Fox } \\
& \text { Skunk Daddy Long LeGamel Bluejay Kangaroo } \\
& \text { Racoon Centipede Zebra Elephant } \\
& \text { Bee Dolphin Fly Tiger Mosquito } \\
& \text { Praying MantisSpider Moose Pig } \\
& \text { Termite Oyster Sheep Narwhal Wolf } \\
& \text { Moth Pigeon Jellyfish Cheetah }
\end{aligned}
$$

D

Conditions

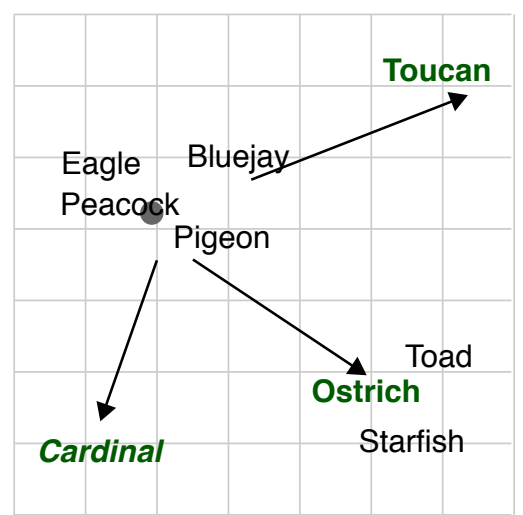

$\mathbf{E}$

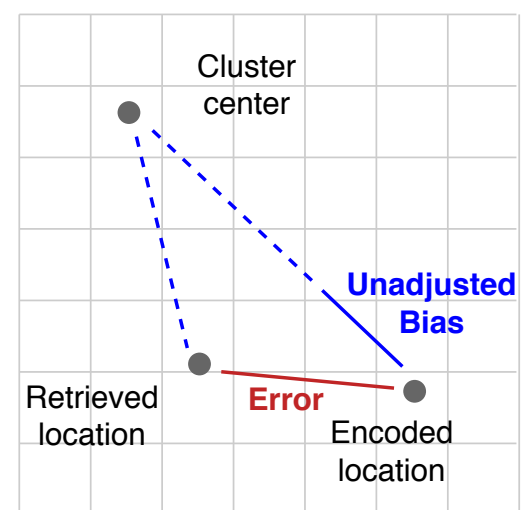

Figure 1 General experiment design (A) Experiment procedure. Participants encoded each imagelocation three times by dragging the image onto a red dot marking its location. After a 5-minute break, participants retrieved the location of each image. (B) Image locations for Experiments 1 and 3 in the experimental groups. (C) Image locations for Experiment 2. (D) Spatial consistency and category typicality for the 'bird' category. Black indicates spatially 'consistent' and green/bold font indicates spatially 'inconsistent' images. All inconsistent images were either typical (italicized) or atypical (not italicized) category members. Gray dot indicates the center of all spatially consistent images in a category. (E) Example of retrieval measures for an image biased towards its category's cluster center. Solid red line indicates error. Solid blue line indicates unadjusted bias. Bias is quantified as an image's unadjusted bias as a proportion of error (solid blue line divided by red line). 


\section{General Methods}

146 Methods described in this section pertain to all four experiments, unless specified below

147 or in the specific methods section for each experiment.

149 Participants

150 Participants completed all experiments on Amazon Mechanical Turk (AMT; see 151 Supplemental Methods for eligibility requirements). The University of Pennsylvania IRB 152 approved all consent procedures. Participants were assigned either to experimental or 153 control conditions.

\section{Materials}

156 Images: Stimuli consisted of $70100 \times 100$-pixel color images of two superordinate 157 semantic categories (animals and objects) on white backgrounds from the Bank of 158 Standardized Stimuli (Brodeur, Dionne-Dostie, Montreuil, \& Lepage, 2010). Piloting was 159 conducted to choose the images in each condition to ensure that high levels of image 160 recognition were matched across the two superordinate categories.

162 Image-location associations: In all experiments, participants learned and retrieved 163 associations between images and locations on a grid. The locations of images were 164 determined by the semantic similarity of the concepts, as assessed by separate cohorts 165 of participants who completed either an odd-man out task (e.g. Connolly, Gleitman, \& 166 Thompson-Schill, 2007), a procedure where participants viewed three images and 167 chose the image that was least similar to the other two (Experiments 1, 3 and 4) or a 
168 typicality ranking task (Experiment 2). Both procedures enabled us to produce a 2169 dimensional array of image locations that closely resembled the relations among 170 concepts in semantic space, clustering according to their category membership. This $2 \mathrm{D}$ 171 mapping was the basis for the image locations in the memory experiments. The center 172 of each category's cluster was derived by computing the mean $\mathrm{x}$ and $\mathrm{y}$ coordinates over 173 all images belonging to that cluster. See the Supplemental Methods for more details 174 about the odd-man-out procedure (Figure S6) and the ranking procedure (Figure S7).

175 In all experiments, we manipulated a subset of images such that their spatial 176 locations no longer corresponded to their category membership (Figure 1D). As an 177 example, in Experiments 1 and 3, the 20\% most typical and 20\% most atypical category 178 members in a category cluster were assigned a new, random location, with the 179 constraint that the location was closer to another cluster center than to its original 180 cluster center. The remaining $60 \%$ of images were left in place, so that participants 181 would be able to learn that different categories were generally associated with a 182 particular spatial location. Thus, in total, 42 images were associated with locations that 183 were consistent with their semantic similarity ('spatially consistent'), and 28 were 184 displaced to a random location ('spatially inconsistent). Of the 28 spatially inconsistent 185 images, 14 were typical category members and 14 were atypical category members. 186 Finally, the displays for animals and objects were arranged on the left and right sides of 187 the screen, randomized for each participant. Thus, locations for most images were 188 spatially consistent with semantic knowledge (e.g. several birds were located in a 189 cluster), but some images were inconsistent (a few birds were located closer to other 190 clusters of animals than to the other birds; Figure 1B-C). 
In each of the four experiments, the above methods were applied to subjects in

192 the experimental group. In the control group, the images paired with each location were

193 shuffled separately for animals and objects such that both groups viewed the same

194 locations, but the images did not cluster by category. In other words, the locations that

195 had originally been associated with spatially (in)consistent or (a)typical category

196 members were associated with random images, rendering these conditions

197 meaningless. This control group ensures that any effects observed in the experimental

198 group are driven by differences in the spatial consistency and category typicality of

199 images relative to their semantic category, rather than idiosyncrasies in the stimulus

200 display (e.g., spatial clustering).

201

202 Procedure

203 Overview: All experiments comprised an encoding phase and a retrieval phase, 204 separated by a 5-minute break when participants were free to complete other tasks or 205 internet browsing (Figure 1A). Before beginning encoding, participants were presented 206 with a white $600 \times 1200$ pixel rectangle with light gray gridlines spaced to form $50 \times 50$ 207 pixel grids. The size of the grid was derived from browser measurements of past AMT 208 participants collected from pilot data to increase the likelihood that a participant could 209 view the entire screen without zooming out. Participants were instructed to adjust the 210 size of their browser, without zooming, so they could see the entire grid. The experiment 211 would not advance until the entire grid was visible. 
213 Encoding: On each trial, participants viewed an image beneath the grid and a red dot on

214 the grid corresponding to that image's location. They were instructed to drag each

215 image onto the dot, to click the mouse button or press the 'enter' key once the image

216 was positioned over the dot, and to try to remember each image's location for a later

217 memory test. After this movement, the participant immediately advanced to the next

218 trial. Trials were self-paced, and participants were unable to proceed to the next trial if

219 they had not moved the image directly over the red dot. To ensure sufficient memory for

220 the locations, all trials were presented three times, in separate blocks. The order of trials

221 was randomized for each participant, separately for each block. The encoding

222 instructions were followed by two practice trials to familiarize participants with the task

223 before beginning encoding.

224

225 Retrieval: The timing and task were identical to those from the encoding phase, but with

226 no red dot marking the location of the image. Participants were instructed to drag the

227 image to its associated location and to make their best guess if they had forgotten it.

228 The trial order was randomized for each participant. In experiments $2-4$, participants

229 also made confidence ratings and in Experiment 3, they also completed an exemplar

230 memory test. These procedures are described in the Methods sections specific to that

231 experiment.

232

233 Statistical analyses

234 We developed two measures to quantify memory accuracy (error) and the influence of 235 category knowledge (bias) for each image (Figure 1E). Error was defined as the 
236 Euclidian distance between an image's encoded location and its retrieved location,

237 where greater values indicate worse memory, and perfect memory would correspond to

238 an error of 0 . Bias was defined as the proportion of total error in the direction of an

239 image's category cluster. To compute this, we first found each image's unadjusted bias

240 by subtracting the Euclidean difference between its encoded location and its cluster

241 center from the Euclidean difference between its retrieved location and cluster center.

242 Then, we divided this unadjusted bias by the amount of error for the image. Thus, a bias

243 score between 0 and 1 indicates that retrieval is biased towards the image's cluster

244 center, and a score between 0 and -1 indicates that retrieval was biased away from the

245 cluster center. These two measures are statistically independent, such that two

246 memories with the same amount of error could differ in whether their retrieval was

247 biased towards or away from their respective cluster centers.

248 Both measures were averaged across trials, by spatial consistency (consistent 249 vs. inconsistent), and then by category typicality (atypical vs. typical). Effects on 250 average error and bias were assessed with two-tailed paired t-tests and repeated 251 measures ANOVAs. We report effect sizes for all significant effects, including partial $\eta^{2}$ 252 for main effects or interactions of ANOVAs and Cohen's $d$ for effect sizes of within253 subject comparisons. The equation used to calculate Cohen's $d$ accounted for the high 254 correlations across these within-subject conditions (Lakens, 2013; Goulet-Pelletier \& 255 Cousineau, 2018):

$$
d=\frac{M_{2}-M_{1}}{\sqrt{S_{1}^{2}+S_{2}^{2}-2 r S_{1} S_{2}}}
$$

$256 M_{1}$ and $S_{1}$ are the mean and standard deviation of one condition, $M_{2}$ and $S_{2}$ are the 257 mean and standard deviation of the other condition, and $r$ is the correlation between the 
258 two. Bootstrapped $95 \%$ confidence intervals for these effect sizes were generated as

259 well. Considering the correlation between conditions results in effect sizes that are more

260 representative of the reported within-subject statistical tests and are comparable across

261 the four experiments reported here, but it is important to note that such effect sizes are

262 inflated compared to effect sizes from between-subject designs and thus may be less

263 generalizable to other experiments in the literature (Lakens, 2013).

264 In Experiments 2 - 4, participants rated their confidence in their memory for the

265 locations, and in Experiments 3, participants also completed an exemplar memory test.

266 Error and bias were analyzed as a function of these behavioral measures. Because the

267 options were not consistently used (e.g. some participants never chose 'Forgot item' as

268 a confidence response), resulting in unequal amounts of data in each confidence bin,

269 linear mixed-effects models were used to investigate how confidence or exemplar

270 memory related to error and bias. Participant intercepts and slope terms for each

271 included predictor variable were modeled as random effects. The significance of a given

272 contrast was obtained using Satterthwaite approximate degrees of freedom, resulting in

$273 F$ or $t$ statistics and corresponding $p$ values. Estimated marginal means (EMMs) were

274 computed to test their simple effects. Tests of simple effects were corrected for multiple

275 comparisons with a Bonferroni correction, depending on the number of tests conducted.

276 Where this correction is applied, the raw p-values are reported alongside the corrected

$277 \alpha$ and number of tests conducted. Figures 3B, 3D, and 4C-D, which visualize the results

278 of mixed-effects models, display averages across trials within participants for each

279 condition for easier interpretation. Because there is no clear precedent for generating

280 standardized effect sizes for mixed-effects models, we computed Cohen's $d$ for all 
281 pairwise contrasts of participants' average error and bias separately at each level of

282 confidence and exemplar memory accuracy (Supplemental Results, Figure S4).

283

284 Table 1. Similarities and differences across the four experiments

\section{$\begin{array}{llll}\text { Experiment } 1 & \text { Experiment } 2 & \text { Experiment } 3 & \text { Experiment } 4\end{array}$}

\begin{tabular}{lllll}
\hline Images & $\begin{array}{l}\text { 70 animals and } \\
\text { objects }\end{array}$ & $\begin{array}{l}160 \text { animals and } \\
\text { objects }\end{array}$ & $\begin{array}{l}70 \text { animals and } \\
\text { objects with } \\
\text { similar lures }\end{array}$ & $\begin{array}{l}70 \text { exemplars of } 6 \\
\text { objects }\end{array}$ \\
\hline Categories & $\begin{array}{l}3 \text { animal and } 3 \\
\text { object categories, } \\
\text { derived from odd- } \\
\text { man-out }\end{array}$ & $\begin{array}{l}4 \text { animal and } 4 \\
\text { object categories, } \\
\text { pre-determined }\end{array}$ & Same as Exp 1 & $\begin{array}{l}3 \text { vehicles and } 3 \\
\text { household } \\
\text { objects, arbitrary } \\
\text { colors }\end{array}$ \\
\hline Typicality & $\begin{array}{l}\text { Derived from odd- } \\
\text { man-out }\end{array}$ & $\begin{array}{l}\text { Derived from list } \\
\text { ranking task }\end{array}$ & Same as Exp 1 & $\begin{array}{l}\text { Distance between } \\
\text { image color and } \\
\text { the center color of } \\
\text { its object set }\end{array}$ \\
\hline
\end{tabular}

Clustered by

\begin{tabular}{|c|c|c|c|c|}
\hline Locations & $\begin{array}{l}\text { Derived from odd- } \\
\text { man-out }\end{array}$ & $\begin{array}{l}\text { categories, } \\
\text { locations evenly } \\
\text { distributed }\end{array}$ & Same as Exp 1 & Same as Exp 1 \\
\hline
\end{tabular}

\begin{tabular}{lllll}
\hline Retrieval & $\begin{array}{l}\text { Drag image to } \\
\text { encoded location }\end{array}$ & Same as Exp 1 & $\begin{array}{l}\text { Choose encoded } \\
\text { image, drag to } \\
\text { location }\end{array}$ & Same as Exp 1 \\
\hline Confidence None & $\begin{array}{l}\text { Rate confidence } \\
\text { of location }\end{array}$ & $\begin{array}{l}\text { Rate confidence } \\
\text { of image } \\
\text { Rate confidence } \\
\text { of location }\end{array}$ & $\begin{array}{l}\text { Rate confidence } \\
\text { of location }\end{array}$ \\
\hline
\end{tabular}

\section{Experiment 1}

287 In Experiment 1, participants encoded image-location associations, where the location

288 of each image was either spatially consistent or spatially inconsistent with its category

289 membership. This experiment was designed to test two predictions: (1) retrieval of an 
290 image's location would be more accurate if its encoded location was spatially consistent

291 with its category membership, by being located near other category members, and (2)

292 for images whose locations were not spatially consistent with their category 293 membership, retrieval of a typical category member would be more biased towards its 294 category's cluster center, relative to an atypical one.

296 Methods

297 Participants: 70 participants were equally divided into the experimental group $(N=35$; 29815 females; mean age $=38.9 ; \mathrm{SD}=10.9$; range $21-71$ years $)$ and the control group $(\mathrm{N}=$ $29935 ; 16$ females; mean age $=37.1 ; S D=10.8$; range 20-61 years). Participants were 300 paid $\$ 5$ upon completion of the experiment.

\section{Results}

303 Spatial consistency: We first tested the prediction that location memory would be more 304 accurate for images that were near its category center relative to images in random 305 locations (Figure 2A). We computed a 2 (group: experimental, control) x 2 (spatial 306 consistency: consistent, inconsistent) ANOVA which revealed a main effect of spatial 307 consistency $F_{(1,68)}=26.96, p<.001, \eta_{p}^{2}=.28$, no main effect of group, $F_{(1,68)}=0.75, p=$ $308.39, \eta_{p}^{2}=.28$, and a significant interaction between group and spatial consistency, $F_{(1,68)}$ $309=18.10, p<.001, \eta_{p}^{2}=.21$. This interaction reflected more error for spatially 310 inconsistent images relative to consistent images in the experimental group, $t_{(34)}=-7.31$, $311 p<.001, d=-1.24$, but not in the control group, $t_{(34)}=-0.61, p=.54, d=-0.10$, where 312 images did not cluster by category (Figure 2B). Location memory was more accurate for 
313 images that were located near their category centers relative to those that were in

314 random locations, suggesting that over the course of the experiment, participants

315 learned that different categories were likely to be located in certain parts of the grid, and

316 the use of this knowledge aided memory for images whose locations matched this

317 spatial organization.

318

319 Category typicality: As a reminder, in cases where the location of an image was not

320 aligned with its category membership, we predicted that retrieval of typical category

321 members would be biased towards their category center (Figure 2C). To test this

322 prediction, we conducted a 2 (group: experimental, control) $\times 2$ (category typicality:

323 typical, atypical) ANOVA with bias of spatially inconsistent images as the dependent

324 variable. We found no reliable main effect of typicality, $F_{(1,68)}=3.57, p=.06, \eta_{p}^{2}=.05$,

325 or group, $F_{(1,68)}=2.06, p=.16, \eta_{p}^{2}=.13$, but a reliable interaction, $F_{(1,68)}=11.69, p=$

$326.001, \eta_{p}^{2}=.15$, (Figure 2D). This interaction reflected greater bias for typical category

327 members relative to atypical category members in the experimental group, $t_{(34)}=3.52, p$

$328=.001, d=0.60$, but not the control group, $t_{(34)}=-1.16, p=.25, d=-0.20$. As predicted,

329 a larger proportion of the error in the placement of typical category members was in the

330 direction of their category clusters, relative to the error of atypical category members.

331 We were concerned that these effects might have arisen simply because typical

332 images were placed more centrally in the grid, which happens to be close to most

333 category clusters, while atypical images were placed at edges of the grid far from the

334 clusters. To test this possibility, we computed each image's average bias towards the

335 two other clusters in the category. A similar group $x$ typicality ANOVA found no main 
A

Error prediction

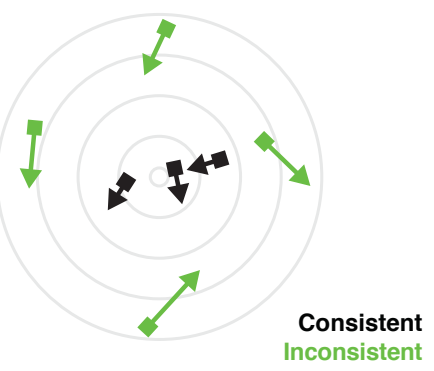

C

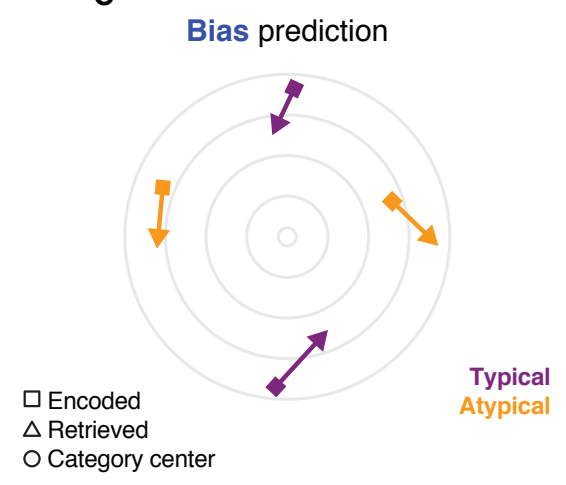

B

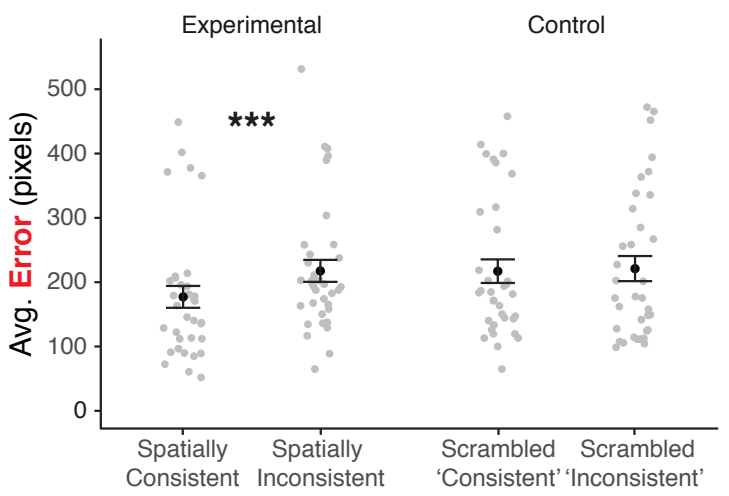

D

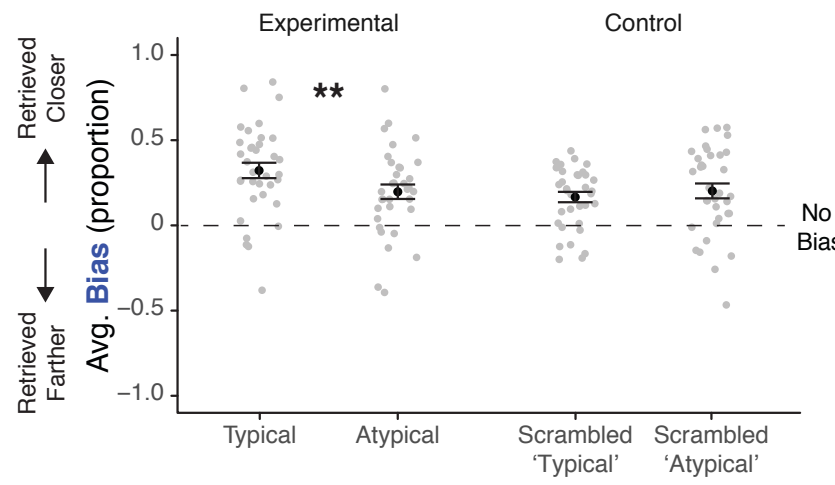

Figure 2 Experiment 1 (A) We predicted that images whose locations were spatially consistent with their category (e.g. located near other category members) would be remembered more accurately than images in random locations. Gray circle indicates the center of a category cluster. Arrows indicate the encoded and retrieved locations of images from that category. Shorter arrows signify more accurate memory. (B) Average error as a function of spatial consistency, where greater values indicate larger errors in location memory. Scrambled 'consistent' and 'inconsistent' conditions in the control group indicate error for images that were randomly assigned to the spatially consistent and inconsistent locations in the experimental group. (C) Of all spatially inconsistent images, we predicted that typical category members would be retrieved closer to their category center relative to atypical members. (D) Average bias by category typicality and group, where $>0$ indicates retrieval closer to the category center than originally encoded and < 0 indicates retrieval away. (B, D) Scrambled 'typical' and 'atypical' conditions in the control group indicate images randomly assigned to the locations of typical and atypical category members in the experimental group. Gray dots indicate participants. Error bars indicate standard error of the mean (SEM) across participants. ${ }^{* *} p<.01 ;{ }^{* *} p<.001$.

After confirming that bias in memory differed according to an image's category typicality, we next explored whether category typicality influenced memory accuracy (Figure S1). To do this, we entered the average error of spatially inconsistent images 
357 into a 2 (group: experimental, control) $\times 2$ (category typicality: typical, atypical) ANOVA.

358 This revealed no main effect of group, $F_{(1,68)}=0.02, p=.88, \eta_{p}^{2}=0.004$, or of typicality,

$359 F_{(1,68)}=0.79, p=.38, \eta_{p}^{2}=.01$, but a reliable interaction, $F_{(1,68)}=4.44, p=.04, \eta_{p}^{2}=.06$.

360 This interaction reflected greater error for typical images relative to atypical images in 361 the experimental group, $t_{(34)}=2.34, p=.03, d=.40$, but not in the control group $t_{(34)}=-$

$3620.79, p=0.43, d=-.13$, suggesting that location memory for atypical category members

363 was more accurate relative to location memory for typical category members. Taken

364 together, this suggests that the structure of a category influenced both the magnitude 365 and direction of error in location memory for images whose spatial locations did not 366 correspond to their category membership.

\section{Discussion}

369 In Experiment 1, we found that participants' retrieval was more accurate for images that 370 were spatially consistent with their category membership, in line with prior observations

371 that new memories are enhanced if they are consistent with prior knowledge. This 372 demonstrated that participants were able to learn that the locations of many images 373 aligned with their category membership and use this knowledge to aid retrieval of 374 images whose locations were consistent with this organization.

375 Furthermore, we observed biases in location memory that reflected the use of 376 this same knowledge of category locations. First, regardless of category membership, 377 images that were located randomly were retrieved closer to their category's cluster 378 center relative to their encoded location. Already, this suggests that participants' 379 reliance on semantic knowledge distorted memory for images whose locations did not 
380 map on the spatial organization that mirrored their category membership. Critically, we

381 also found variation in the extent to which these memories were distorted. When sorting

382 the spatially inconsistent images by category membership, typical category members

383 were more biased towards their category neighbors relative to atypical ones. This

384 finding provides novel evidence that variations in the structure of semantic knowledge,

385 like category membership and typicality, can govern the extent of distortion in new 386 episodic memories.

\section{Experiment 2}

389 Category membership and typicality in Experiment 1 were derived using a data-driven 390 procedure, based on semantic judgments provided by a separate cohort of participants. 391 We developed Experiment 2 as a conceptual replication with three goals. First, we 392 aimed to investigate whether we would observe the same effects using conditions that 393 were derived from a more validated procedure for identifying typical and atypical 394 category members. Second, we aimed to rule out the possibility that memory was more 395 accurate for spatially consistent images because their spatial locations were more 396 densely clustered, increasing the likelihood of guessing the correct location. Replicating 397 the effects observed in Experiment 1 with these changes would confirm that these 398 effects generalize across different task parameters and an expanded set of images.

399 Third, we added a confidence measure to understand how the strength of 400 memory for each image location may modulate its influence by prior knowledge. Past 401 work has shown that weaker memory for specific events are more likely to draw on 402 other sources of memory. One consequence of this reconstruction process is that less 
403 confident memories are prone to more error and are more biased by category 404 information (Persaud \& Hemmer, 2016; Brady, Schacter, \& Alvarez, 2018). We 405 predicted that, if participants drew on a combination of memory of specific locations and 406 knowledge of category locations, they would rely more on category knowledge when 407 memories for specific locations were weak. This would result in (1) a larger difference in 408 accuracy for spatially inconsistent versus consistent images for low-confident memories, 409 and (2) more biased retrieval, where low-confident images would be retrieved closer to 410 their category center (Brady et al., 2018). As we did not have strong predictions about 411 whether confidence would differentially relate to the observed typicality effects, we 412 conducted an exploratory analysis of their interaction.

\section{Methods}

415 Participants: 70 participants took part in Experiment 2, half in the experimental group (N $416=35 ; 14$ females; mean age $=38.8 ; \mathrm{SD}=10.4$; range $=22-70$ years) and half in the 417 control group $(\mathrm{N}=35 ; 16$ females; mean age $=38.3 ; \mathrm{SD}=11.8$; range $=24-72$ years $)$. 418 Participants were paid $\$ 10$ upon completion of the experiment.

420 Materials: Stimuli comprised 160 100x100-pixel color images on white backgrounds (80 421 animals, 80 objects). These 2 superordinate categories were each divided into 4 422 categories with 20 images each: birds, insects, sea creatures, mammals, clothes, 423 furniture, kitchen, and office. The categories were selected from prior studies of 424 categorization norms (Deyne et al., 2008; Uyeda \& Mandler, 1980). Category typicality 425 was determined with a list ranking procedure completed by a separate group of 
426 participants. See Supplemental Methods and Figure S7 for details of this procedure and

427 the resulting rankings.

428 To generate images' locations, the memory grid was divided into halves with 429 animals on one side, and objects on the other. On each side, all images were spaced 430 uniformly apart, resulting in an even distribution of images across the grid. Each side's 431 locations were divided into four quadrants, and the four categories were randomly 432 assigned to different quadrants.

433 Like in Experiment 1, the spatial locations of a subset of images were 434 manipulated such that their locations no longer were consistent with semantic 435 knowledge (Figure 1C). To do this, the 15\% most typical and 15\% most atypical 436 category members were swapped with the typical and atypical members of other 437 categories such that each quadrant had an equal number of typical and atypical images 438 from the other three categories. The remaining $70 \%$ of images were randomly assigned 439 to locations within their category's quadrant. In total, 112 images were in locations that 440 were spatially consistent with their category membership ('consistent'), and 48 were 441 associated with a random location ('inconsistent). Of the 48 inconsistent images, 24

442 were typical and 24 were atypical category members. The sides (animals on right or left) 443 and quadrants were randomized for each participant.

444

445 Procedure: The timing, task, instructions, and statistical analyses were identical to those 446 of Experiment 1, with the exception of an added confidence measure. After each 447 retrieval trial, participants chose from four options to report the confidence in their 
448 location memory: 'Very confident', 'Somewhat confident', 'Guessed', and 'Forgot item'.

449 Clicking on an option automatically advanced the participant to the next trial.

\section{$451 \quad$ Results}

452 Spatial consistency: Like in Experiment 1, we first computed a 2 (group: experimental, 453 control) $\times 2$ (spatial consistency: consistent, inconsistent) ANOVA to test whether spatial 454 consistency with category knowledge influenced the accuracy of location memory. This 455 revealed a main effect of consistency, $F_{(1,68)}=38.63, p<.001, \eta_{p}{ }^{2}=.36$, and no main 456 effect of group, $F_{(1,68)}=0.01, p=.91, \eta_{p}{ }^{2}=.008$. There was a significant interaction 457 between group and consistency, $F_{(1,68)}=35.33, p<.001, \eta_{p}^{2}=.34$, explained by greater 458 error for spatially inconsistent images relative to spatially consistent images in the 459 experimental group, $t_{(34)}=-7.35, p<.001, d=-1.24$, but not in the control group, $t_{(34)}=-$ $4600.24, p=.81, d=-0.04$ (Figure 3A). Replicating the observations in Experiment 1, 461 retrieval was more accurate for images located close to their category center.

We next sought to investigate how error was modulated by participants' reported 463 confidence. Focusing on the experimental group, we entered consistency and 464 confidence of each trial into a mixed-effects model with error as the dependent 465 measure. We found a strong interaction between spatial consistency and confidence, $466 F_{(3,1522.36)}=3.14, p=.02$, in addition to significant main effects of consistency, $F_{(1,49.27)}$ $467=38.77, p<.001$, and confidence, $F_{(3,46.90)}=83.18, p<.001$ (Figure 3B). The main 468 effect of confidence reflected less error for more confident responses relative to less 469 confident responses for all pairwise comparisons of confidence responses (all $t<-3.5$, 470 all $p<.002, \alpha=.008$ with Bonferroni correction for 6 tests). Pairwise tests of error by 
471 consistency for each confidence response revealed that the difference in error by

472 consistency is greater only for less confident memories (very confident: $t_{(398.18)}=-0.52, p$

$473=.60$; somewhat confident: $t_{(100.17)}=-6.13, p<.001$; guessed: $t_{(119.95)}=-5.58, p<.001$;

474 forgot item: $t_{(663.30)}=-3.70, p<.001 ; \alpha=.0125$ with Bonferroni correction for 4 tests),

475 confirming our prediction of a greater use of category knowledge when retrieving

476 weaker memories relative to strong ones.

477

478 Category typicality: As in Experiment 1, we examined how category typicality modulated 479 bias in memory, again predicting that retrieval of typical category members would be 480 more biased towards their category center than retrieval of atypical category members. 481 We conducted the same 2 (group: experimental, control) $\times 2$ (category typicality: typical, 482 atypical) ANOVA of bias for spatially inconsistent images. This analysis revealed a 483 significant interaction between group and typicality, $F_{(1,68)}=4.20, p=.04, \eta_{p}^{2}=.06$, in 484 addition to a main effect of group, $F_{(1,68)}=6.61, p=.01, \eta_{p}^{2}=.28$, and no main effect of 485 typicality, $F_{(1,68)}=0.78, p=.38, \eta_{p}^{2}=.01$ (Figure 3C). As in Experiment 1, the interaction 486 reflected more bias for typical category members relative to atypical category members 487 in the experimental group, $t_{(34)}=2.14, p=.04, d=.36$, but not the control group, $t_{(34)}=-$ $488 \quad 0.80, p=.43, d=-0.14$.

We also investigated how confidence modulated bias with a linear mixed effects 490 linear model of confidence and typicality as predictors, focusing only on the 491 experimental group (Figure 3D). This analysis revealed a main effect of confidence, $F(3$, $49248.69)=10.04, p<.001$. There was no reliable effect of typicality, $F_{(1,75.45)}=0.22, p=.64$, 493 and no significant interaction between typicality and confidence, $F_{(3,1033.28)}=2.09, p=$ 
494 .10. To understand the main effect of confidence, we computed comparisons across 495 confidence responses, collapsed over typicality $(\alpha=.008$, Bonferroni correction for 6 496 tests). We found that 'very confident' responses were less biased towards their cluster 497 center relative to 'somewhat confident': $t_{(23.29)}=-3.87, p<.001$, and relative to 498 'guessed': $t_{(28.46)}=-3.0, p=.006$. Interestingly, 'forgot item' responses were less biased 499 relative to 'guessed' responses, $t_{(18.66)}=2.76, p=.01$, and relative to 'somewhat 500 confident' responses: $t_{(22.61)}=2.89, p=.009$, but these comparisons do not survive 501 correction for multiple comparisons.

502 We next aimed to replicate the influence of category knowledge on the accuracy 503 of spatially inconsistent images observed in Experiment 1 . We conducted the same 2 504 (group: experimental, control) $\times 2$ (category typicality: typical, atypical) ANOVA of 505 average error for spatially inconsistent images (Figure S1). This revealed no effect of 506 group, $F_{(1,68)}=1.24, p=.27, \eta_{p}^{2}=.19$, and a reliable main effect of typicality, $F_{(1,68)}=$ $5079.65, p=.003, \eta_{p}^{2}=0.12$. This was qualified by an interaction between typicality and 508 group, $F_{(1,68)}=5.77, p=.02, \eta_{p}^{2}=.08$, such that there was greater error for typical 509 category members relative to atypical ones in the experimental group $t_{(34)}=3.44, p=$ $510.002, d=.58$, but not in the control group $t_{(34)}=0.59, p=.56, d=.10$. As in Experiment 511 1, memory for the location of typical category members was less accurate than that of 512 atypical category members. 
A

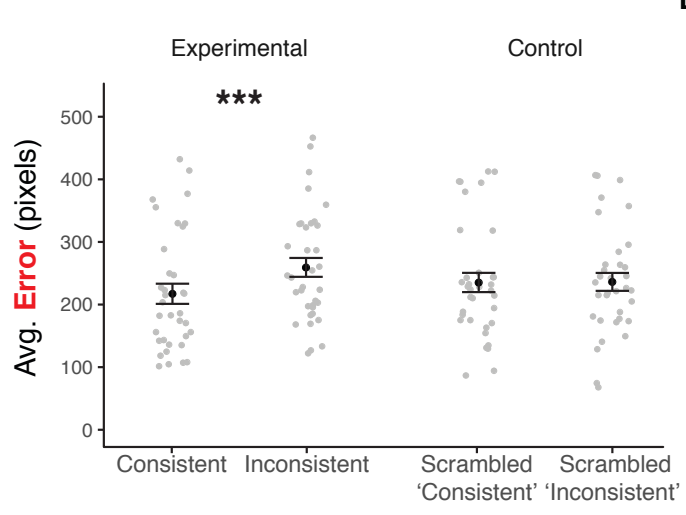

B

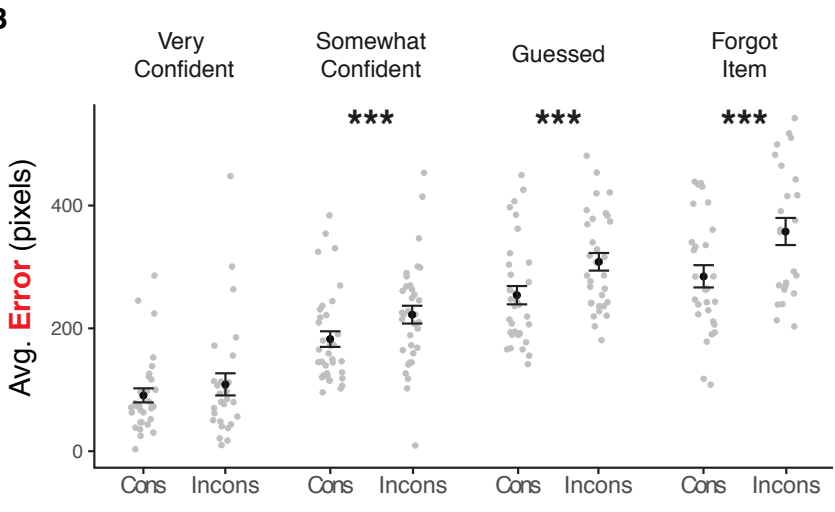

C

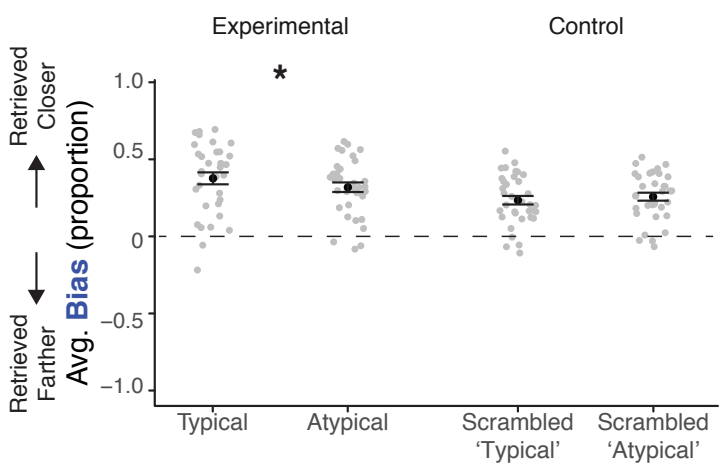

D

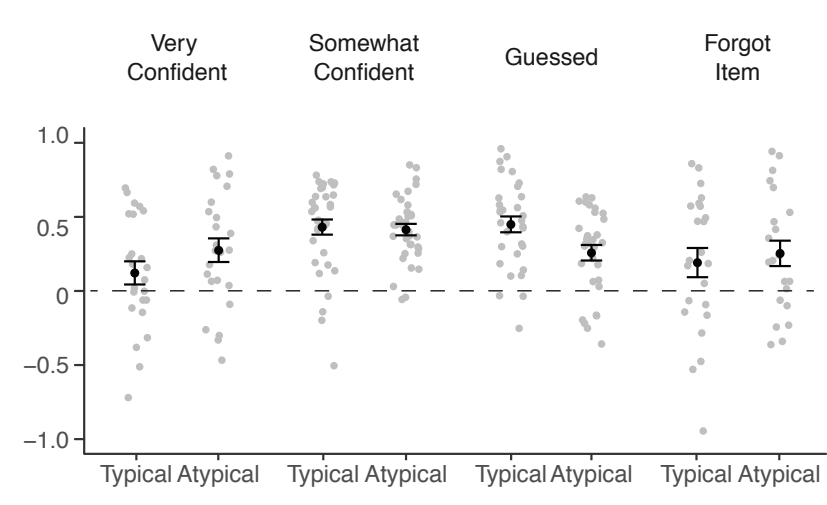

\section{Discussion}

526 In Experiment 2, we replicated and extended the findings of Experiment 1. Across both

527 experiments, participants were able to learn that images of a certain category were

528 likely to appear in the same area of the grid, and this knowledge affected their retrieval

529 of specific image locations. First, memory was more accurate if the images spatially
Figure 3 Experiment 2 (A) Average error by spatial consistency, where greater values indicate less accurate location memory. (B) Average error by spatial consistency and reported confidence in the experimental group. Statistics reflect the simple effects of a mixed-effects model. (C) Average bias of spatially inconsistent images by typicality and group, where $>0$ indicates that trials were retrieved closer typicality and reported confidence in the experimental group. Statistics reflect results of trial-level mixedeffects models. (A-D) Gray dots indicate participants. Error bars indicate standard error of the mean (SEM) across participants. Scrambled conditions in the control group indicate retrieval of the images at locations to which spatially (in)consistent and (a)typical images had been assigned in the experimental group; these locations were randomly assigned images in the control group. ${ }^{*} p<.05 ;{ }^{* * *} p<.001$. 
531 far from their category cluster, participants made systematic errors: memory for typical 532 category members was less accurate than for atypical ones, and a larger proportion of 533 their error was in the direction of their category's cluster center. These results were 534 observed in both experiments despite differences in the numbers and types of 535 categories, method of determining typicality, and mapping between category 536 membership and spatial location. These observations provide strong evidence for the 537 theory that episodic retrieval is supported by integrating over multiple sources of 538 information: memory for specific image-location associations, and knowledge of 539 categories and their mapping to a general location. This integration can either benefit or 540 harm memory for individual images, depending on whether these sources are in conflict. 541 The introduction of a confidence measure provided further support for this theory.

542 First, we found that for low-confident responses, error was more likely to be modulated 543 by spatial consistency such that images located far from their cluster center were 544 remembered less accurately than those located near their cluster center. However, as 545 confidence in location memory increased, there was a less of a difference in error by 546 consistency. This suggests that to retrieve stronger episodic memories, there is less of

547 a need to rely on other information, like knowledge of the clustering of the images by 548 category. When focusing on spatially inconsistent images, we found that low-confident 549 memories were more biased towards category neighbors relative to high-confident 550 memories, again consistent with the notion that less strong episodic memories will be 551 more distorted by other sources of knowledge (Brady et al., 2018). The finding that 552 memory was less biased for images which were forgotten entirely is puzzling; this effect 
553 should be interpreted with caution as this option was used the least frequently and

554 consequently is the most under-powered and most variable condition in this analysis.

\section{Experiment 3}

557 Experiments 1 and 2 suggest that the way that prior knowledge is organized can shape

558 how new episodic memories are formed and retrieved. In particular, we find that prior 559 knowledge biases new memories differently based on their category typicality. How 560 does the presence of category information as an organizing structure during learning 561 give rise to this bias? One possibility is that because typical category members are 562 more strongly associated with other category members relative to atypical ones, 563 interacting with a typical category member automatically brings to mind richer category 564 information, resulting in less of a need to deeply encode or attend to its idiosyncratic 565 features. This difference in processing may make encoding of typical category members 566 more efficient, but at a cost to memory for other features that are unique to the encoded 567 event.

568 This line of reasoning was inspired by observations that stimuli are often better 569 remembered when they violate or are irrelevant to a salient task structure (Goodman, 570 1980; Love, Medin, \& Gureckis, 2004; Sakamoto \& Love, 2004; Bejjani \& Egner, 2019).

571 Moreover, distinct features of a stimulus can be processed differently according to 572 whether the features align with the task structure. Evidence for this idea can be found in 573 experiments where participants learned that certain facial attributes dictated a face's 574 spatial location. Location memory was better for faces with these attributes, but memory 575 for other facial attributes was impaired, suggesting that the presence of a guiding 
576 organization during encoding aided memory for features that adhered to the

577 organization at the cost of memory for idiosyncratic features (Sweegers \& Talamini,

578 2014; Sweegers, Coleman, van Poppel, Cox, \& Talamini, 2015). If typical images in the

579 present experiments are thought of as more adherent to the organizational properties

580 that determine category membership, and thus are less deeply processed, we would

581 predict that memory for their idiosyncratic features may be impaired relative to that of 582 atypical category members.

583 We tested this prediction by assessing whether memory for a different feature of 584 the stimuli is modulated by category typicality. To do this, we adapted the procedure in 585 Experiment 1 by adding an exemplar memory test. During retrieval, participants were 586 tested for their exemplar memory by choosing between the encoded image and a highly 587 similar perceptual lure. They then dragged the chosen image to its encoded location. 588 We predicted that exemplar memory for typical category members would be worse than 589 for atypical members in the experimental group, where category membership was 590 related to most images' locations and thus was a salient feature for participants to learn 591 during encoding. In the control group, where participants encoded the same images but 592 in random locations, we expected no difference in exemplar memory by typicality.

593 We also conducted exploratory analyses to understand how exemplar memory 594 interacted with location memory. Like exemplar memory, the location associated with 595 each image can be thought of as an idiosyncratic feature of memory for the animals and 596 objects, because the locations are newly learned and specific to the context of the 597 experiment. Importantly, for spatially inconsistent images, this feature is in direct conflict 598 with the organizational scheme guiding image locations. Already, our findings in 
599 Experiments 1 and 2 are consistent with this notion when considering the location of the 600 spatially inconsistent images as an idiosyncratic detail. Memory for the locations of

601 typical category members was less accurate and more biased than memory for 602 locations of atypical category members, perhaps because their locations were less 603 deeply encoded. Thus, we predicted that poor exemplar memory would be related to 604 even stronger bias for typical category members, as memory for both features may be 605 correlated within each image-location association.

\section{Methods}

608 Participants: 230 participants took part in Experiment 3, divided into the experimental 609 group $(\mathrm{N}=115 ; 44$ females; mean age $=35.3 ; \mathrm{SD}=8.4$; range $=22-63$ years $)$ and the 610 control group $(\mathrm{N}=115 ; 55$ females; mean age $=36.7 ; \mathrm{SD}=10.0$; range $=19-67$ years $)$. 611 See Supplemental Methods for the procedure used to determine the number of 612 participants recruited. Participants were paid $\$ 5$ upon completion of the experiment.

614 Materials: Experiment 3 was designed to be as similar as possible to Experiment 1 , 615 while adding exemplar memory and confidence measures during retrieval. Thus, 616 encoded stimuli consisted of 70 color images on white backgrounds ( 35 animals, 35 617 common objects) and these stimuli were shown during retrieval along with 35 animal 618 lures and 35 object lures. The images were taken from a variety of public datasets 619 (Brodeur et al., 2010; Konkle \& Caramazza, 2013; Russo et al., 2018; Stark, Yassa, 620 Lacy, \& Stark, 2013). The 70 animals and objects presented during encoding 621 corresponded to those in Experiment 1, but the images representing each animal and 
622 object were replaced by images drawn from the datasets published by Stark and

623 colleagues, which feature perceptually matched pairs of images commonly used in

624 behavioral studies examining pattern separation (Kirwan, Jones, Miller, \& Stark, 2007).

625 If an image from Experiment 1 did not have a corresponding pair of images in this

626 database, a second image was found from a different database or from the internet and

627 matched with the one used in Experiment 1. Piloting conducted with 35 pilot participants

628 demonstrated that there was no difference in exemplar memory between typical and

629 atypical images and that accuracy for a given image was not at ceiling or at floor.

631 Procedure: The timing, task, and instructions were identical to that of Experiment 1, 632 except for the retrieval task. On each retrieval trial, a similar lure appeared next to the 633 encoded image (right/left presentation randomized). Participants were instructed to 634 choose the image they had encoded, drag it to its location, and confirm their answer by 635 clicking continue or pressing the space bar (Figure 4A). Then, participants completed 636 two confidence measures. First, they assessed confidence for the image they chose 637 ('Very confident', 'Somewhat confident', 'Completely guessed'). Next, they assessed 638 confidence of their retrieved location ('Very confident', 'Somewhat confident', 639 (Completely guessed'). See the Supplemental Results for analyses of exemplar 640 memory, error (Figure S2B), and bias (Figure S3B) by confidence.

\section{Results}

643 Exemplar memory: We first tested the prediction that the image details of atypical 644 category members would be more deeply encoded than those of typical category 
645 members. To do this, we compared exemplar memory as a function of category 646 typicality. As a reminder, we predicted that a difference in exemplar memory by 647 typicality would only be present in the experimental group, where category membership 648 was related to most images' locations and thus was a salient feature for participants to 649 learn and use. We quantified exemplar memory as the proportion of trials in which 650 participants chose the correct image. Note that in this analysis, in contrast to all other 651 analyses conducted up to this point, typicality in the control group refers to the true 652 typicality of the category members, not of the images that were located in the positions 653 assigned to typical category members in the experimental group.

654 As we predicted, we found that atypical category members were more accurately 655 recognized than typical ones in the experimental group, $t_{(114)}=2.96, p=.004 d=.28$, 656 but not in the control group, $t_{(114)}=0.94, p=.35, d=0.09$ (Figure 4B). When comparing 657 across groups, the experimental group exhibited better exemplar memory for atypical 658 category members, $t_{(228)}=-3.10, p=.002, d=-0.41$, and to a lesser extent for typical 659 ones $t_{(228)}=-1.95, p=.05, d=-0.26$. However, a 2 (group: experimental, control) $\times 2$ 660 (typicality: typical, atypical) ANOVA did not reveal a significant interaction between 661 group and typicality, $F_{(1,228)}=1.95, p=.16, \eta_{p}^{2}=.008$, so differences in exemplar 662 memory by typicality across the groups should be interpreted with caution. This ANOVA 663 also revealed a significant main effect of group, $F_{(1,228)}=8.07, p=.005, \eta_{p}^{2}=.12$, and of 664 typicality, $F_{(1,228)}=7.48, p=.007, \eta_{p}^{2}=.03$. In summary, when images were presented 665 in a context where category membership was relevant to the location memory task, 666 participants exhibited better memory for the image details of atypical category members 667 relative to typical ones. 
669 Spatial consistency: Before considering how exemplar memory influenced location

670 memory accuracy, we first confirmed that retrieval of spatially consistent images was

671 more accurate than retrieval of images in random locations, replicating our observations

672 in Experiments 1 and 2 (see Supplemental Results). We next explored whether

673 exemplar memory and consistency interacted to influence accuracy, focusing on the

674 experimental group. To do this, we computed a mixed-effects linear model with error as

675 the dependent variable and spatial consistency, exemplar memory accuracy, and their

676 interaction as independent variables (Figure 4C). Exemplar memory reliably related to

677 error, $F_{(1,123.6)}=20.57, p<.001$. There was no reliable interaction between exemplar

678 memory and consistency, $F_{(1,6178.8)}=3.55, p=.06$. In post-hoc comparisons $(\alpha=.0125$

679 when Bonferroni-corrected for 4 tests), we found that for spatially inconsistent images, 680 error was reliably greater when exemplar memory was incorrect relative to when 681 exemplar memory was intact, $\mathrm{t}_{(430.92)}=-4.32, p<.001$, while for spatially consistent 682 images, this difference did not survive correction for multiple comparisons $t_{(234.77)}=-$ $6832.35, p=.02$. At the same time, there was greater error for spatially inconsistent relative 684 to consistent images regardless of exemplar memory accuracy (exemplar memory 685 correct: $t_{(187.92)}=-8.14, p<.001$, exemplar memory incorrect: $\left.t_{(955.07)}=-7.10, p<.001\right)$.

686 In some, exemplar memory accuracy was related to location memory only for spatially 687 inconsistent images, with worse performance on the exemplar test relating to less 688 accuracy in location memory. 
690 Category typicality: After replicating the observation from Experiments 1 and 2 that 691 typical category members were retrieved closer to their category center than atypical 692 category members (see Supplemental Results), we next tested whether exemplar 693 memory and typicality interacted to influence bias in location memory (Figure 4D). We 694 predicted that typical category members with poor exemplar memory would be more 695 biased than ones with intact exemplar memory. We also conducted exploratory 696 comparisons of bias by typicality separately for correct and incorrect exemplar memory $697(\alpha=.0125,4$ tests). Contrary to our prediction, we found no reliable difference in bias by 698 exemplar memory accuracy, neither for typical category members, $t_{(306.43)}=0.38, p=$ 699.70 , nor atypical category members $t_{(316.27)}=-0.86, p=.39$. When considering images 700 that were correctly remembered, retrieval was more biased for typical relative to atypical 701 category members, $t_{(192.84)}=4.52, p<.001$. In contrast, there was no reliable difference 702 of typicality for images with incorrect exemplar memory, $t_{(830.02)}=1.64, p=.10$. 703 However, we found no interaction between exemplar memory and typicality, $F_{(1,3069.36)}=$ $7040.87, p=.35$, so differences in bias by category typicality should be interpreted with 705 caution. Finally, there was no reliable main effect of exemplar memory, $F_{(1,101.27)}=0.12$, $706 p=.73$. In sum, when participants remembered the details of the images, typical images 707 were retrieved closer to their category center relative to atypical images, but when 708 participants forgot the exemplar, this difference in bias by category typicality was 709 attenuated. 


\section{Retrieval Instructions}

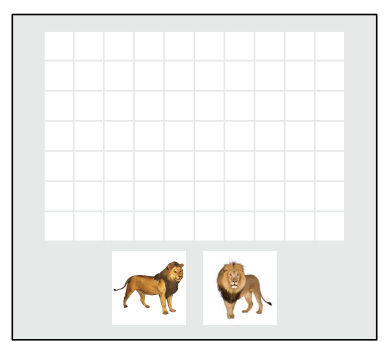

Drag the studied image to its location
B
Control

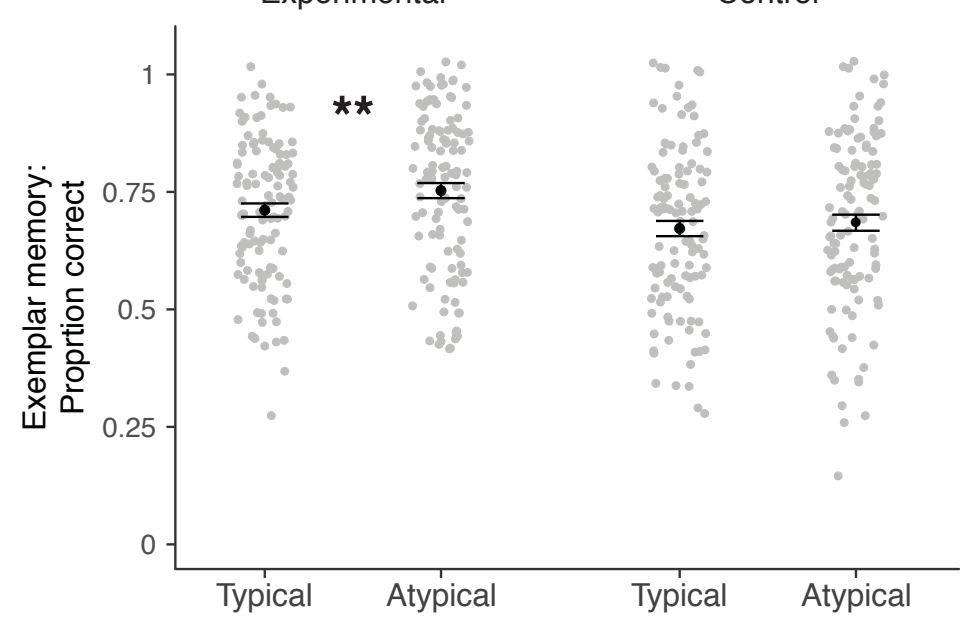

C

Figure 4 Experiment 3. (A) Retrieval instructions and display. (B) Performance on the exemplar memory test by group and category typicality. (C) Average error in the experimental group, by spatial consistency and by performance on the exemplar memory test (correct versus incorrect). Statistics reflect results of trial-level mixed-effects models. (D) Average bias by typicality and performance on the exemplar memory test in the experimental group, where values $>0$ indicate that trials were retrieved closer to neighbors than originally encoded and values $<0$ indicate retrieval away from neighbors. (B-D) Statistics reflect simple effects that survive a Bonferroni correction for 4 comparisons $(\alpha=0.0125)$. Gray dots indicate participants. Error bars indicate SEM. ${ }^{* *} p<0.01 ;{ }^{* * *} p<0.001$.

722 led to differences in how participants remembered typical and atypical category

723 members. We found that, when category information was salient and useful during 
724 encoding, there was better memory for the image details of atypical category members

725 over typical ones. This suggests that the idiosyncrasies of typical category members are

726 more poorly remembered than those of atypical category members, consistent with the

727 notion that typical category members may be processed less deeply and more

728 efficiently than atypical ones. Critically, this difference in processing occurred only in an

729 encoding context where category information was made more salient by the associated

730 spatial configuration. In the control group, where category information did not dictate the

731 locations of images, there was no difference in exemplar memory for typical and

732 atypical category members. Note that while these results are consistent with our

733 predictions, the lack of a reliable interaction prevents us from strongly interpreting these

734 differences in exemplar memory across the two groups, and more work is needed to 735 provide evidence for this interpretation.

736 We also replicated the observation in Experiments 1 and 2 that images located in

737 random locations were less accurately remembered relative to images located close to

738 their category center. Furthermore, this difference in memory accuracy was magnified

739 for images with incorrect exemplar memory, although the interaction between spatial

740 consistency and exemplar memory was not statistically reliable. This gives rise to an

741 intriguing possibility that for spatially inconsistent images, exemplar accuracy is a

742 marker for the fidelity of each image-location association in that incorrect exemplars

743 accompanied less accurate location memory. Alternatively, exemplar memory did not

744 strongly modulate location memory for spatially consistent images, perhaps because

745 participants could rely on prior knowledge to guide retrieval regardless of the fidelity of

746 their memory of the specific image and its location. 
Interestingly, exemplar memory also influenced how category typicality

748 influenced bias, with stronger bias towards category neighbors for typical category

749 members relative to atypical ones, but only for images with intact exemplar memory.

750 Bias was not reliably affected by typicality for images with incorrect exemplar memory.

751 Notably, we chose our sample size based on a power analysis that included estimates

752 of exemplar memory accuracy, expecting that there would be fewer trials with incorrect

753 exemplar memory and any comparisons of typicality by accuracy would be less

754 powered when limited to trials with incorrect exemplar memory. Thus, we are confident

755 that this analysis was sufficiently powered to identify differences by typicality for trials

756 with incorrect exemplar memory. However, counter to our prediction, this difference in

757 bias was not driven by changes in memory for the typical category members, as there

758 was no indication of increased bias for typical category members with incorrect

759 exemplar memory over ones with intact exemplar memory. We speculate that typical

760 and atypical images with incorrect exemplar memory were similarly poorly encoded,

761 with less processing of their category typicality, and thus retrieval of their locations was

762 equally prone to influence by category neighbors. For stronger memories, as indicated

763 by correct exemplar memory, retrieval of typical images was more biased than that of

764 atypical images, suggesting a difference in processing that protects atypical category

765 members from the influence of category neighbors. Indeed, while not significant, the

766 retrieval of atypical category members was numerically less biased when their exemplar 767 was correctly remembered relative to when it was forgotten.

768 Interestingly, how memory for details of the exemplars affects bias seems 769 different from how reported confidence relates to bias, although both measures can be 
770 thought of as markers of memory strength. In this experiment as well as in Experiment

7712 , confidence in location memory did not interact with category typicality to influence

772 bias (see Supplemental Results, Figure S3A-B). In contrast, images with intact

773 exemplar memory exhibited a robust difference in bias by typicality, while images whose

774 specific exemplar was forgotten did not. We discuss this potential discrepancy in more

775 depth in the General Discussion.

776 In summary, we find that encoding in the context of a particular organizational

777 structure alters memory for individual exemplars, in particular for details that are

778 orthogonal or in opposition to the salient structure: memory for specific images and for

779 random locations. Together, these results provide a possible explanation for why we

780 observe differences in bias for location memory for typical and atypical category

781 members, and opens up new testable questions about the relationship between the use

782 of prior knowledge and the depth or efficiency of new encoding.

783

Experiment 4

785 In the prior three experiments, we found that location memory was more biased by

786 category knowledge for typical category members relative to atypical ones. What can

787 such biases tell us about category typicality? One possibility is that images of typical

788 category members are more strongly influenced by other category members because

789 they are more strongly associated; for example, retrieval of typical words is more

790 clustered than retrieval of atypical words in a free recall task (Bousfield, Cohen, \&

791 Whitmarsh, 1958). Alternatively, typical category members may look more similar to

792 other category members, and this increased visual similarity may make it easier to 
793 confuse the locations of typical category members with other images that happen to be

794 near its cluster center. This explanation would not require prior semantic knowledge, but

795 rather would apply to any stimuli that vary in visual similarity.

796 Experiment 4 aimed to adjudicate between these two possibilities. We developed

797 a set of stimuli whose organization mimicked that of the categories employed in

798 Experiments $1-3$, but category typicality was determined by arbitrary assignment of 799 colors to different manmade objects (Figure 5A). As an example, instead of using a 800 superordinate category (e.g., birds) that comprised different basic level concepts (e.g., 801 robins and eagles), in Experiment 4 a basic level category (e.g., lamps) comprised 802 multiple exemplars of the concept (e.g. different styles of lamps), with an arbitrarily 803 assigned color for the category (e.g. all lamps had a greenish hue). The color of each 804 exemplar image was sampled from a distribution (e.g., each lamp could be more or less 805 green). The exemplars assigned colors from the ends of this distribution were labeled 806 'atypical' and the exemplars assigned colors from the center of this distribution were 807 labeled 'typical'. Thus 'typical' exemplars looked more similar to other category 808 members relative to 'atypical' exemplars because they were closer in color. Note that 809 this was the only sense in which the images were typical or atypical; the assignment of 810 a particular exemplar to a specific color was randomly determined.

811 If typical images are retrieved closer to their similarly-colored category neighbors

812 relative to atypical images, that would provide support for the notion that biases due to 813 category typicality observed in Experiments $1-3$ are driven in part by the visual

814 similarity of features across different category members. It would also provide support 815 for the notion that biases in memory can be driven by newly-learned, arbitrary 
816 mappings, like colors assigned to manmade objects, rather than stable semantic

817 information, like knowledge of animal taxonomy and object function.

818

\section{Methods}

820 Participants: 70 participants took part in Experiment 4, either in the experimental group

$821(\mathrm{~N}=35 ; 17$ females; mean age $=34.6 ; \mathrm{SD}=8.1 ;$ range $=22-61$ years $)$ or the control

822 group $(\mathrm{N}=35 ; 12$ females; mean age $=34.2 ; \mathrm{SD}=9.6 ;$ range $=25-64$ years $)$.

823 Participants were paid $\$ 5$ upon completion of the experiment.

824

825 Materials: Stimuli consisted of 70 100x100-pixel images on white backgrounds (35 826 vehicles, 35 household objects; Brady et al., 2018). To mimic the six categories in 827 Experiments $1-3$, the images comprised exemplars of three vehicles (planes, trains 828 and cars) and three household objects (chairs, lamps, and clocks).

829 To mimic variance in category typicality, we created variance in how similar 830 object exemplars were within each category by changing their colors (Figure 5A). First, 831 we assigned each category a central color by choosing six equidistant points around a 832 360-degree color space (i.e. each value separated by $60^{\circ}$ ). We then generated six 833 normal distributions $\left(\sigma=20^{\circ}\right)$ around these points. The width of these normal 834 distributions ensured that the range of colors assigned to each category covered a 835 largely unique color range. Next, we pseudo-randomly sampled from each of these 836 distributions to assign each image to a unique color that fell within its category's color 837 range. Images with color values farthest from their category's central color were 838 'atypical' category members, and images with colors closest to their category's central 
839 color were 'typical' category members. To ensure that the range of colors assigned to 840 spatially (in)consistent and (a)typical conditions was consistent across categories, the

841 normal distribution for each category was divided into 5 bins, where the breaks between

842 bins captured cumulative probabilities bounded by the following z-statistics: [inf -1.405 -

8430.2020 .2021 .405 inf]. Colors for atypical images were sampled from the two bins that

844 comprised the tails of the distribution, colors for typical images were sampled from the

845 bin comprising the center of the distribution, and colors for all spatially consistent

846 images were evenly sampled from the two remaining bins.

847 To ensure that any differences in category typicality were driven by color and not

848 by other perceptual features of the images, the assignment of the six color ranges to the 849 six categories and the assignment of colors to images within each category were 850 randomized separately for 6 counterbalancing groups (See Supplemental Methods). In 851 other words, the 6 groups viewed different combinations of image-color associations 852 and different image exemplars associated with typical and atypical colors, minimizing 853 the possibility that any observed influences of color typicality would be driven by 854 idiosyncrasies of the other features of the images.

855 The number and locations of images in each category were exactly matched to 856 the memory displays derived for Experiment 1 . Specifically, locations that belonged to 857 the bird category in Experiment 1 were assigned a color category with the same number 858 of category members, and the locations of the (a)typical birds were now assigned 859 (a)typically-colored images. Thus, in addition to learning the locations of individual 860 exemplars of an object, participants could learn that the object corresponded to a range 861 of colors and a general location on the grid. Spatially 'consistent' images were located 
862 near images of the same object in the same color range, and 'inconsistent' images were

863 in random locations. Critically, analogously to Experiments 1 and 2, the colors of half of

864 the spatially inconsistent images were 'typical' or sampled from the center of that 865 category's color distribution, and the colors of the other half of the images were 866 'atypical', or sampled from the tails of the category's color distribution.

868 Procedure: The timing, task, and instructions were identical to that of Experiment 1, with

869 the identical confidence measure from Experiment 2. See the Supplemental Results for 870 analyses of error (Figure S2C) and bias (Figure S3C) by confidence.

871

\section{Results}

873 Spatial consistency: We first examined whether the same differences in accuracy by

874 spatial consistency observed in Experiments 1 - 3 would extend to stimuli organized by 875 object type and color (Figure 5B). In line with those experiments, we predicted that 876 memory would be more accurate for spatially consistent images relative to inconsistent 877 ones (e.g. green lamps that were located near each other versus ones that were in 878 random locations). A 2 (group: experimental, control) x 2 (spatial consistency: 879 consistent, inconsistent) ANOVA revealed a significant main effect of consistency, $F_{(1,68)}$ $880=76.87, p<.001, \eta_{p}^{2}=.53$, and no reliable main effect of group, $F_{(1,68)}=3.68, p=.06$, $881 \eta_{p}^{2}=.40$. Critically, the ANOVA also revealed an interaction between group and 882 consistency, $F_{(1,68)}=16.52, p<.001, \eta_{p}{ }^{2}=0.20$. Like in Experiment 3 , both the 883 experimental group, $t_{(34)}=-7.78, p<.001, d=-1.32$, and control group, $t_{(34)}=-4.16, p<$ $884.001, d=-0.70$, exhibited greater error for spatially inconsistent images relative to 
885 consistent images, suggesting that the dense clustering of images in spatially consistent 886 locations may have aided retrieval in both groups, but this difference in error by spatial 887 consistency was greater for the experimental group relative to the control group. This 888 suggests that the organization of images by object and color further aided memory for 889 images whose locations were consistent with that spatial organization.

891 Color ('category') typicality: As a reminder, our critical prediction was that retrieval of 892 typically colored images would be more biased towards the similarly colored exemplars 893 of the same object relative to atypically colored images. This finding would be 894 analogous to the biases due to category membership seen in the first three 895 experiments. However, there was no reliable difference in bias for typical images 896 relative to atypical images in the experimental group, $t_{(34)}=1.46, p=.15, d=0.25$, or in 897 control group, $t_{(34)}=0.76, p=.45, d=.13$ (Figure 5C). Furthermore, a 2 (group: 898 experimental, control) $\times 2$ (typicality: typical color, atypical color) ANOVA did not reveal 899 a main effect of typicality, $F_{(1,68)}=2.38, p=.13, \eta_{p}{ }^{2}=.03$, or a significant typicality by 900 group interaction, $F_{(1,68)}=0.17, p=0.68, \eta_{p}^{2}=.002$. Interestingly, this analysis revealed 901 a main effect of group, $F_{(1,68)}=18.75, p<.001, \eta_{p}^{2}=0.51$, with more bias regardless of 902 category typicality in the experimental group relative to the control group, $t_{(68)}=4.33, p<$ $903.001, d=1.04$. Taken together with the error analyses, this suggests that when images 904 were organized by color and object type, the retrieval of spatially inconsistent images 905 was biased towards the center of their object clusters, even though their accuracy did 906 not differ from subjects whose images were not organized by object type and color. 
As typically-colored and atypically-colored images did not differ in the proportion

908 of error that was biased towards their category cluster, it may seem like the color 909 manipulation simply was not effective, and that participants did not meaningfully encode 910 the colors of each image or use color information during retrieval. However, we 911 uncovered one piece of evidence that this manipulation may have had a limited effect.

912 When considering an image's unadjusted bias, we found that typically-colored images 913 were retrieved closer to their category center relative to atypically-colored images in the 914 experimental group, $\mathrm{t}_{(34)}=2.05, \mathrm{p}=.048, d=.35$, and not in the control group, $\mathrm{t}_{(34)}=-$ $9150.30, p=.76, d=-.05$. However, there was no reliable interaction between group and 916 typicality, $F_{(1,68)}=1.91, \mathrm{p}=.17, \eta_{\mathrm{p}}{ }^{2}=0.03$. While not robust, this provides some 917 evidence that participants attended to the colors of each image during encoding and 918 used this information to a limited degree during retrieval.

919 Finally, for comparison to Experiments 1 - 3, we also examined location memory 920 accuracy among the spatially inconsistent images to understand how the typicality of an 921 image's color, relative to the mean color of its object category, influenced bias in 922 location memory (Figure S1). As a reminder, retrieval of typical category members was 923 less accurate than retrieval of atypical category members in the prior experiments. We 924 asked whether there were analogous differences in error by the 'typicality' of the colors 925 assigned to each image. A 2 (group: experimental, control) by 2 (typicality: typical color, 926 atypical color) ANOVA revealed no reliable main effects or interaction between typicality 927 and group, all $F<0.49$, all $p>0.48$. The lack of an effect suggests that memory 928 accuracy was not influenced by the typicality of an image's color. 
A

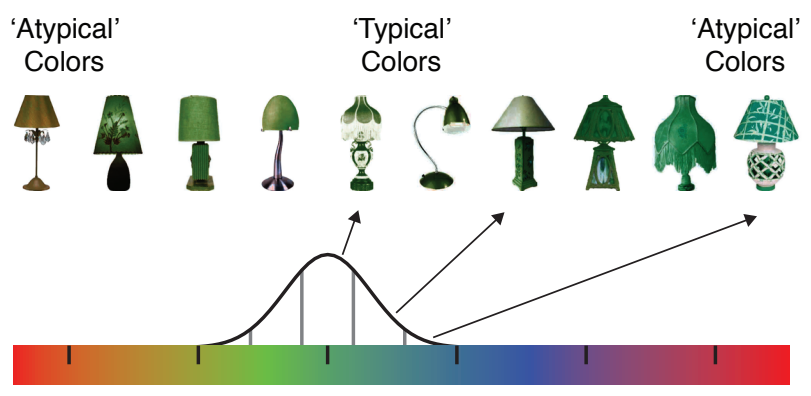

B

Experimental Control
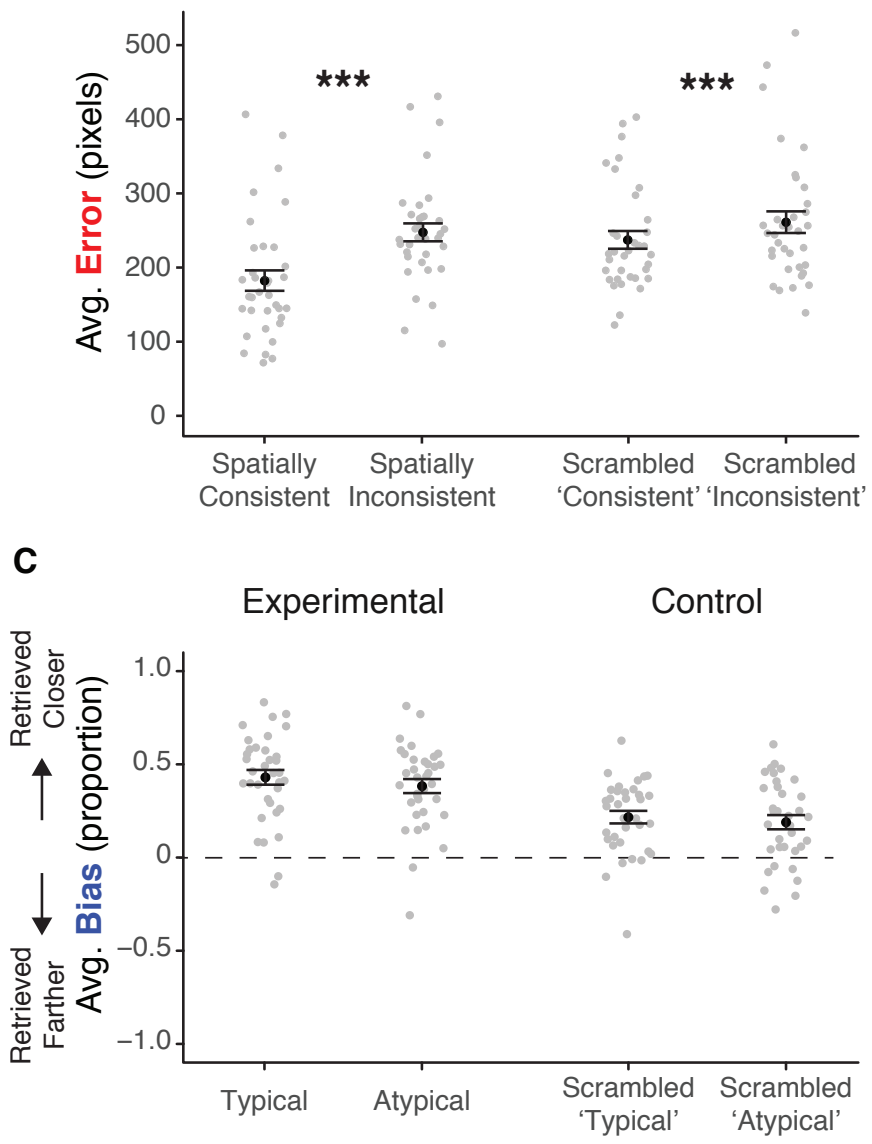

930 Figure 5 Experiment 4. (A) Typicality determined by color. The six vertical lines on the color bar indicate of 'lamp'. Typical and atypical lamps reflect the distance between their color and the color at the center of the lamp distribution. (B) Average error by spatial consistency and group. (C) Average bias by color typicality and group. Gray dots indicate participants. Error bars indicate SEM. ${ }^{* \star *} p<.001$. 


\section{Discussion}

936 The aim of Experiment 4 was to understand whether the biases in memory by category

937 typicality observed in Experiments $1-3$ could be driven by visual similarity of the 938 images. Over the course of encoding specific image-location associations, participants 939 also learned that exemplars of a certain type of object, like lamps, tended to be located 940 in certain areas of the screen and associated with a certain color. This organization 941 influenced the retrieval of specific images, such that images in random locations were 942 retrieved less accurately. Note that differences in accuracy here could be driven either 943 by images clustering by object type, by color, or by a combination of the two.

944 The critical comparison was of bias in retrieval based on whether the arbitrary 945 colors of encoded objects were more or less similar to the mean color of each object 946 set. This continuous variation in color within an object set mirrors the graded manner in

947 which category members vary in their similarity to a central tendency or prototype of 948 each category. Analogously to the typical and atypical category members in spatially 949 inconsistent locations in Experiments $1-3$, images whose colors were closest or 950 farthest from the mean color of that object were randomly located. We found weak 951 evidence that typically colored images were retrieved closer to their category clusters, 952 but only when employing a different bias measure that did not account for error. 953 Moreover, in contrast to Experiments 1 - 3, color typicality did not influence accuracy or 954 bias by their category clusters. This suggests that visual similarity alone cannot explain 955 the bias effects observed in Experiments 1 - 3. Rather, bias in retrieval in Experiments $9561-3$ may be driven primarily by the strength of associations between category 957 members, but also in small part by their visual similarity. 
One reason why visual similarity did not robustly influence memory may be that

959 the task was more difficult than in Experiments $1-3$, since participants needed to

960 encode the locations of many exemplars of the same object sets, rather locations of

961 unique animals and objects. Differences in difficulty could also explain the strong main

962 effect of group on bias in this experiment, where the experimental group exhibited more

963 bias relative to the control group regardless of category typicality. However, accuracy in

964 Experiment 4 was not reliably worse than in Experiment 1 when averaging across all

965 conditions (see Comparisons Across Experiments), which suggests that Experiment 4

966 was not meaningfully more difficult. Another possibility is that the feature that gave

967 structure to each category was based purely on color, which was newly learned and

968 arbitrarily assigned. In contrast, images in Experiments 1 - 3 were organized by multiple

969 features of animals and objects that likely were learned long before participants began

970 the experiment. Moreover, features that give rise to visual similarity may also be

971 correlated with other dimensions of similarity. For example, a cardinal and a bluejay

972 look similar because they both have wings, a perceptual feature that also serves the

973 same function. Thus, differences in images' typicality by color may have been less well

974 encoded and thus did not bias retrieval as strongly as in the prior experiments.

975

976

\section{Comparisons Across Experiments}

977 The four experiments varied in the number of images and categories and the manner in

978 which their locations were organized. In the following section, we provide a direct

979 comparison of effects across the four experiments. 
981 Average error: First, to understand whether the details of each experiment induced 982 differences in their difficulty, we investigated whether average error across all images 983 differed across the four experiments. We computed a 2 (group: experimental, control) $\mathrm{x}$ 9844 (experiment: $1-4$ ) ANOVA with average error as the dependent variable. This 985 analysis revealed no main effect of experiment, $F_{(3,432)}=1.01, p=.39, \eta_{p}^{2}=0.007$, no 986 main effect of group, $F_{(1,432)}=1.99, p=.16, \eta_{p}{ }^{2}=0.005$, and no group by experiment 987 interaction, $F_{(3,432)}=0.70, p=.55, \eta_{p}^{2}=0.005$. This suggests that the average memory 988 accuracy across all images was not reliably different across the four experiments or 989 across the experimental and control groups, despite many differences across the 990 experiments.

991

992 Error by spatial consistency: We next investigated whether the relationship between 993 accuracy and spatial consistency with category knowledge differed across the four 994 experiments. To do this, we first created a difference score for each participant to 995 capture the extent to which their accuracy was influenced by spatial consistency. To do 996 this, we subtracted their average error for spatially consistent images from their average 997 error for spatially inconsistent images, where greater values indicate a larger difference 998 in error by spatial consistency. We then computed a 2 (group: experimental, control) x 4 999 (experiment: $1-4)$ ANOVA with this difference score as the dependent variable. This 1000 analysis revealed a strong main effect of group, $F_{(1,432)}=86.04, p<.001, \eta_{p}^{2}=0.17$, 1001 reflecting the greater difference in error by spatial consistency for the experimental 1002 group relative to the control group that was observed in all four experiments. There was 1003 also a main effect of experiment, $F_{(3,432)}=6.27, p<.001, \eta_{p}^{2}=0.04$, and no group by 
1004 experiment interaction, $F_{(3,432)}=1.12, p=0.34, \eta_{p}^{2}=0.008$. The main effect of 1005 experiment reflected a larger difference in error by consistency in Experiment 4 relative 1006 to Experiments $1-3$, all $t>3.01$, all $p<.003$, and no difference amongst Experiments 1 $1007-3$, all $t<0.61$, all $p>.54$, when collapsing across the experimental and control groups. 1008 As a reminder, in Experiments 1 - 3, participants learned that most members of a 1009 category tended to be clustered in the same general location, while in Experiment 4, 1010 most exemplars of the same object within the same color range were clustered. As 1011 participants in Experiment 4 needed to discriminate and encode many exemplars of an 1012 object, it may be that participants' overall memory in this experiment was driven less by 1013 object and color information and more by the uneven density of spatial locations present 1014 in both the experimental and control groups. However, the lack of a reliable interaction 1015 suggests that the extent to which memory accuracy was governed by an image's spatial 1016 consistency was not meaningfully different across the four experiments. See Figure 6A 1017 for a comparison of standardized effect sizes for each group and experiment.

1019 Bias by typicality: We next asked whether there were differences in bias by category 1020 typicality across the four experiments. To do this, we computed a difference score to 1021 capture the extent to which each participant differed in their bias by category (or color) 1022 typicality. To do this, we subtracted their average bias for atypical category members 1023 from their average bias for typical ones, such that positive values indicate more bias for 1024 typical relative to atypical category members. We then entered these difference scores 1025 into a 2 (group: experimental, control) x 4 (experiment: 1 - 4) ANOVA. This analysis 1026 revealed the expected main effect of group, $F_{(1,432)}=21.37, p<.001, \eta_{p}^{2}=0.05$, and no 
1027 reliable main effect of experiment, $F_{(3,432)}=0.63, p=0.60, \eta_{p}^{2}=0.004$, or group by 1028 experiment interaction, $F_{(3,432)}=1.36, p=0.26, \eta_{p}^{2}=0.009$. This suggests that despite 1029 the numerous differences across the four experiments, the extent to which the 1030 organizational structure of the images biased memory for specific images did not 1031 meaningfully differ. See Figure 6B for a comparison of effect sizes of bias by typicality 1032 for both the experimental and control groups in each experiment.

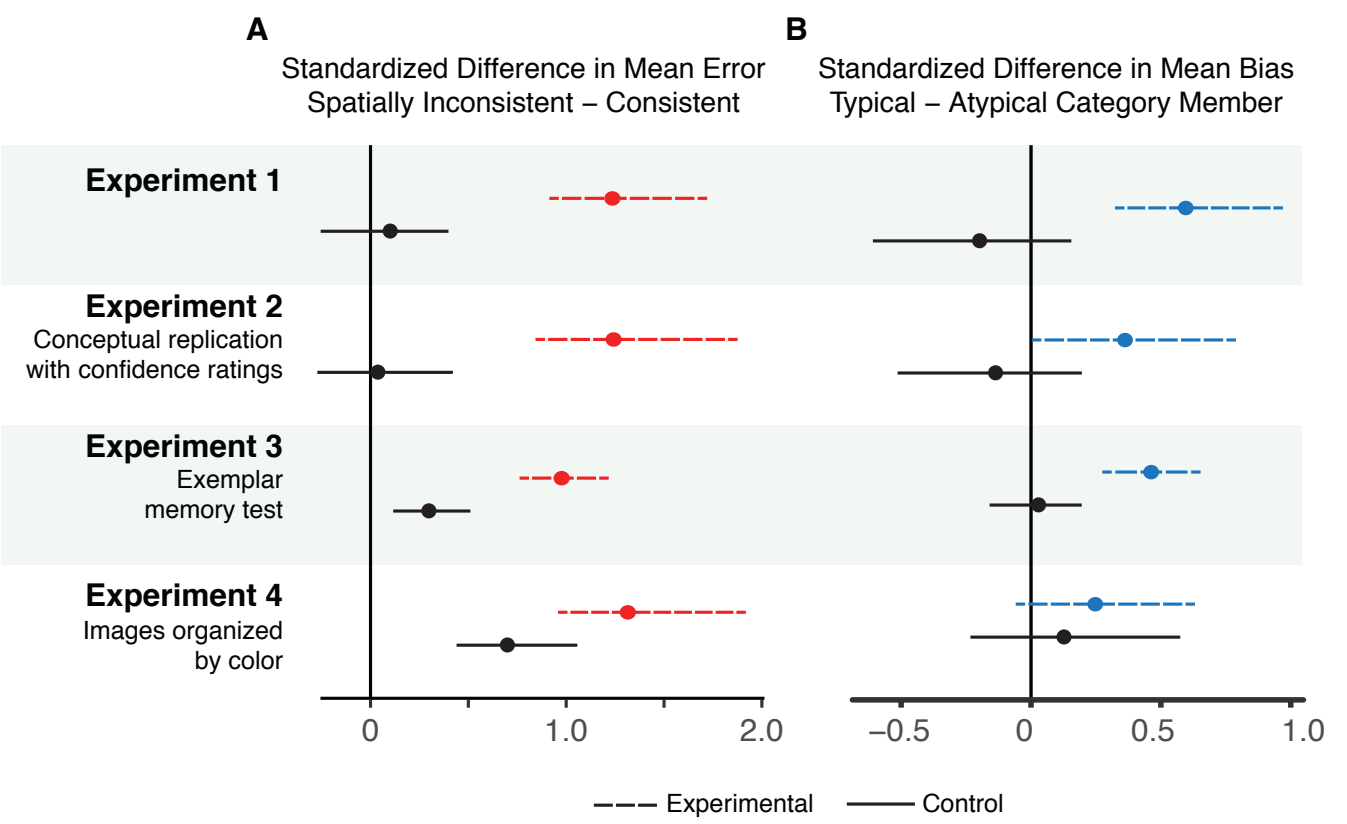

1033

1034

1035

1036

1037

1038

1039

1040

1041

1042

1043

1044

Figure 6 Effect sizes across experiments. (A) Standardized difference in mean error for spatially consistent images versus spatially inconsistent images. (B) Standardized difference in mean bias for typical versus atypical images. Points represent mean effect size and lines represent bootstrapped 95\% confidence intervals. 0 on the $\mathrm{x}$-axis reflects no difference in error by consistency or bias by typicality.

\section{General Discussion}

Across four experiments, we aimed to understand how variations in the organization of semantic knowledge governs distortions in new episodic memories. To do this, we developed a novel location memory task, where participants encoded associations between images and locations. Critically, most images were spatially clustered by their category membership, such that participants could learn associations between an 
1045 image's category and its spatial location, but a minority of images were located in 1046 random locations. Using a reconstruction framework, we predicted that retrieval of

1047 episodic memories may be supported by a mixture of recalled details of the original 1048 event as well as more general knowledge. Here we operationalized 'general knowledge' 1049 as the learned locations for each category of images, which could only be used if 1050 participants recruited their semantic knowledge of animal and object categories as they 1051 encoded specific image-location associations. Indeed, we found that manipulating the 1052 alignment between new memories and general knowledge influenced both the accuracy 1053 and distortion of new memories. First, memory was more accurate for images located 1054 near others from the same category, confirming learning of the category-location 1055 associations. Second, for images that were in random locations, participants made 1056 systematic errors: More typical category members were retrieved as closer to neighbors 1057 of the same category than atypical category members. These results replicated across 1058 multiple experiments despite differences in the number and type of categories 1059 presented, method of determining typicality, specific images used, and mapping 1060 between category membership and spatial location. Below, we discuss these findings

1061 through the lens of memory reconstruction, position our observations of category 1062 typicality in the context of research on the structure of semantic memory, and consider 1063 some limitations of the current design and opportunities for future work.

\section{Memory as reconstruction}

1066 Our main findings provide strong evidence for the theory that episodic retrieval is a 1067 reconstructive process, rather than a reproductive process. In other words, the retrieval 
1068 of episodic memories is not veridical, but instead is supported by the integration of 1069 different sources of information, including (but not limited to) memory for the details of 1070 the event, prior knowledge relevant to the event, and one's expectations about what 1071 may or may not have happened (Bartlett, 1932; Schacter \& Addis, 2007). In this section, 1072 we discuss several of our observations in terms of this framework: why some memories 1073 are more easily remembered than others, why some memories are systematically 1074 distorted, and how prior knowledge interacts with memory strength, operationalized 1075 here as reported confidence.

1076 First, if a newly experienced event is consistent with prior knowledge, combining 1077 the details of that event with prior knowledge is thought to enhance its retrieval by 1078 reducing noise. In our experiments, an image located near category neighbors could be 1079 retrieved by combining memory for its location with knowledge about the general 1080 location of images from the same category, resulting in more accurate retrieval of these 1081 images' associated locations. In contrast, for images in random locations, this same 1082 integration would result in greater errors because the image's location conflicts with the 1083 locations of much of its category. Such findings are consistent with a large and diverse 1084 body of work showing that prior knowledge facilitates new memory formation and 1085 retrieval (Posner \& Keele, 1968; Bransford \& Johnson, 1972; Craik \& Lockhart, 1972; 1086 Schulman, 1974). Our findings extend these results by showing that prior knowledge 1087 can improve encoding of new, unrelated features of a concept. In our experiments, 1088 participants mapped images onto spatial locations on a grid. These locations were not 1089 intrinsically related to the images (e.g., nothing about the concept of a 'spatula' implies 1090 that it should be located on the top right corner of a grid). However, by associating these 
1091 locations with the semantic organization of the images, participants learned to treat 1092 location as a new 'feature' of the image associated with its category membership. Thus, 1093 prior knowledge can help to organize the encoding of unrelated contextual details.

1094 Another prediction from this memory reconstruction framework is that systematic 1095 errors can occur when there is conflict between prior knowledge and the formation of 1096 new memories. In these cases, integration of these two sources of information would 1097 result in a distorted memory that is somewhere between the veridical event and what 1098 would have been expected from prior knowledge. Indeed, it is well known that episodic 1099 memories can be easily distorted by many sources of information, including retroactive 1100 interference, gist-based false memory driven by interference with the surrounding 1101 context, and more (Loftus \& Palmer, 1974; Roediger \& McDermott, 1995). Additional 1102 evidence for distortion in memory comes from the use of continuous reports, which 1103 allows retrieval to be broken down into item-specific error and systematic influences of a 1104 particular category or structure (Huttenlocher et al., 1991; P. Hemmer \& Steyvers, 2009; 1105 Persaud \& Hemmer, 2016). This work demonstrates that new memories can be biased 1106 towards similar stimuli, for example, that memory for the color of an object is biased 1107 towards a canonical color. We extended this work by showing that, in contexts where 1108 semantic knowledge can aid the formation of new memories for spatial locations, 1109 memory for these locations was biased towards category members. Moreover, while we 1110 had strong predictions that category typicality would influence the direction of error, we 1111 also explored whether such influences would also result in differences in accuracy, or 1112 absolute error independent of its direction. Interestingly, by assessing error in memory 1113 as a function of typicality, we also found that typical category members were less 
1114 accurately remembered in Experiments 1, 2, and 3 (Figure S1). The fact that typical

1115 images were both less accurate and more biased by category neighbors opens up

1116 interesting new questions for future research about the relationship between different

1117 sources of error in memory. At the same time, both findings provide evidence for the

1118 notion that semantic knowledge can exert an influence on new memory formation.

1119 A memory reconstruction framework also considers the relative strengths of prior 1120 knowledge and memory for specific events, such that retrieval of an event will rely more

1121 on prior knowledge if the strength of the event memory is weak relative to when it is

1122 strong (Brady et al., 2018). This may explain observations that prior knowledge only

1123 begins to influence new memory after a delay (Lewis \& Durrant, 2011; Payne et al., 1124 2012; van Kesteren, Rijpkema, Ruiter, \& Fernández, 2013). One possibility for the 1125 increasing influence of prior knowledge on new memory over time is that as the details 1126 of the specific events decay, general knowledge is more strongly weighted in the 1127 reconstruction of the memory. In Experiments 2 and 3, we found an interaction between 1128 confidence and the spatial consistency of image locations with their category 1129 membership, such that the benefit of semantic knowledge (i.e. increased accuracy for 1130 spatially consistent images versus inconsistent images) was weaker for images whose 1131 locations were confidently remembered. In the same experiments, we found that more 1132 confident memories were less biased towards category members. Both observations 1133 would be predicted by the integration of specific memories and semantic knowledge in 1134 situations where specific memories are weak and prior knowledge is strong, providing 1135 additional support for the memory reconstruction framework. 
Although a reconstruction framework can be used to formalize how we can

1137 integrate over any number and type of information sources to support memory, it is

1138 particularly valuable for understanding interactions between episodic and semantic

1139 memory. Although the distinction between semantic and episodic memory proposed by

1140 Tulving (1972) has long shaped their separate examination, there is ample evidence of

1141 interactions between the two systems, including demonstrations that newly learned

1142 associations can prime lexical judgments (McKoon \& Ratcliff, 1979) and that the order

1143 in which a list of studied words is recalled is affected by their semantic similarity 1144 (Howard \& Kahana, 2002). These and many other findings call into question whether

1145 the two systems are separate (Anderson \& Ross, 1980; Greenberg \& Verfaellie, 2010;

1146 McKoon, Ratcliff, \& Dell, 1986) and highlight the importance focusing on the similarities

1147 and differences in the processes that underlie both systems (e.g. Cox, Hemmer, Aue, \&

1148 Criss, 2018). By demonstrating that the use of semantic knowledge can systematically

1149 aid or distort memories for new events, our results add support to the notion that the two

1150 systems are constantly interacting. We take this one step farther by demonstrating that

1151 separate semantic and episodic elements of the same event can interact to inform

1152 memory retrieval. In our experiments, each trial was composed of multiple 'episodic'

1153 elements, including the visual features of an image and its spatial location, and

1154 'semantic' elements, including the image's category membership and typicality.

1155 Separately manipulating and tracking variations in these elements afforded us a unique 1156 opportunity to expand our understanding of memory reconstruction by investigating how

1157 complex semantic structures like category membership may influence new episode 
1158 formation, and by assessing episodes composed of multiple features that could 1159 differently vary in memory strength, both of which are discussed in more detail below.

1160 One important avenue for future work is to understand when this reconstruction 1161 process supports memory. For instance, prior knowledge may shape how a new 1162 experience is encoded by combining event-specific and general knowledge at the time 1163 the specific event is experienced. Alternatively, event-specific and general knowledge 1164 may be separately encoded and then integrated during retrieval. Interestingly, past work 1165 has provided evidence for both mechanisms. The reconstruction theory posits that bias 1166 emerges during retrieval, when integrating the noisy, but unbiased, details of the 1167 experience with other prior knowledge (Pernille Hemmer \& Persaud, 2014). 1168 Alternatively, prior knowledge may not distort memory for the event itself but instead 1169 constrains its search space during retrieval, such that retrieval is biased towards other 1170 category members because the locations near other category members are the most 1171 likely options to choose from. At the same time, however, there is also evidence that 1172 prior knowledge shapes encoding, as it can enhance reading comprehension and 1173 various perceptual tasks (Posner, Goldsmith, \& Welton Jr, 1967; Bransford \& Johnson, 1174 1972; Ratcliff \& McKoon, 1995), which likely do not rely on retrieval computations. 1175 Similarly, neuroscientific evidence suggests prior knowledge influences both encoding 1176 and retrieval of new memories. Recent work in humans and rodents has revealed a role 1177 for the hippocampus and prefrontal cortex when encoding experiences that are related 1178 to prior knowledge (Tse et al., 2011; van Kesteren, Fernández, Norris, \& Hermans, 1179 2010; Bein, Reggev, \& Maril, 2014), but also when retrieving them (van Kesteren, 1180 Rijpkema, Ruiter, \& Fernández, 2010). Taken together, this suggests that prior 
1181 knowledge likely plays an important role at both encoding and retrieval. Pinpointing

1182 when biases in new memories emerge may benefit from causal manipulations that can

1183 isolate encoding and retrieval computations.

1184 An important feature of our design is that retrieval could be quantified as a 1185 continuous variable, allowing us to quantify both the direction and magnitude of error for 1186 each trial. However, this measurement is unable to disentangle the types of error in 1187 memory that underpin the observed differences in bias by category typicality; for 1188 instance, bias in memory may be characterized as either a relatively small distortion, 1189 where error is minimal but systematically influenced by category knowledge, or as a 1190 swap error, where bias towards an image's category center is due to conflating memory 1191 for its location with the location of another image near the cluster center. It seems likely 1192 that location memory for typical category members would be more likely to be swapped 1193 in such a manner, thus explaining the increased bias towards the category center. 1194 Although we did not originally design the reported experiments to adjudicate between 1195 these possibilities, we attempted to characterize participants' retrieval in a manner that 1196 captured both types of error by binning each retrieved location as a function of the 1197 relative distance between its encoded location and cluster center (Figure S5). We found 1198 that across all experiments in which stimuli were organized by category, retrieval was 1199 closer to their encoded locations than to their respective cluster centers. Although 1200 typical category members were overall more likely to be retrieved closer to their 1201 category center relative to atypical ones, they were still most likely to be retrieved closer 1202 to their actual encoded locations. While this exploratory analysis should be interpreted 1203 with caution, it hints at the possibility that the difference in bias by category typicality 
1204 was driven by small differences in the magnitude of error, rather than qualitative 1205 differences in how these category members were retrieved.

\section{Possible explanations for differential bias by category typicality}

We chose to employ category typicality as a means to quantify how semantic

1209 knowledge influences new memories, as typicality underscores many neural and

1210 behavioral phenomenon (Patterson, 2007), including decision response times and

1211 recognition (E. H. Rosch, 1973; E. Rosch et al., 1976) as well as variation in deficits in

1212 semantic dementia (Bozeat et al., 2003; Rogers, Patterson, Jefferies, \& Lambon Ralph,

1213 2015). More importantly, category typicality directly influences new memory formation.

1214 When encoding lists of exemplars from a certain category, typical category members

1215 are more likely to be recalled (Schmidt, 1996) and more likely to be falsely inserted

1216 when they are not included in the list (Roediger \& McDermott, 1995; Smith, Ward,

1217 Tindell, Sifonis, \& Wilkenfeld, 2000). Of particular relevance are observations of

1218 asymmetries in linking new information to typical and atypical category members. For

1219 instance, fast mapping, a phenomenon by which learning the name of a novel object is

1220 facilitated by the presence of a known foil (Carey \& Bartlett, 1978; Coutanche \&

1221 Thompson-Schill, 2014) is more likely to occur when the foil is a typical image relative to

1222 an atypical image (Coutanche \& Koch, 2017). Similarly, asymmetries by typicality are

1223 seen when generalizing new knowledge about a category. In these category-based

1224 induction experiments, participants are more likely to attribute new properties of an

1225 exemplar to members of the same category if the exemplar is a typical category

1226 member (Rips, 1975; Osherson et al., 1990; Dunsmoor \& Murphy, 2014). Our 
1227 experimental manipulation demonstrates a complementary effect - having acquired new

1228 knowledge about a category (e.g. most birds are located in a certain part of the grid)

1229 participants were more likely to distort their memory of typical images such that their

1230 locations complied more with this general knowledge.

1231 Upon observing that category typicality explained variance in distortions in new

1232 memories, we developed Experiments 3 and 4 in order to understand what properties of

1233 semantic memory drive such distortions. One possibility is that relative to atypical

1234 category members, typical category members are more strongly influenced by the

1235 location of members of the same category because they are more densely connected,

1236 and activating a typical category member is more likely to activate information about the

1237 rest of the category (Anderson, 1983). A spreading activation account would mirror

1238 observations that participants are more likely to cluster the recall of typical category

1239 members relative to atypical ones (Bousfield et al., 1958). In our experiment, greater

1240 spread of activation for typical category members would mean that learning about a

1241 typical category member's location would activate more representations of category

1242 members with conflicting locations. As a result, their retrieval be more prone to

1243 interference from these conflicting locations. Alternatively, because typical category

1244 members by definition share more features with others (E. Rosch \& Mervis, 1975), they

1245 may be more confusable, such that locations of typical category members may have

1246 been more likely to be swapped for the location of a category neighbor that happened to

1247 be near its cluster center. This explanation could be applied to any set of stimuli that

1248 vary in visual similarity. Indeed, in an experiment where many exemplars of a category

1249 (e.g. different backpacks) were assigned colors sampled from a distribution around a 
1250 central color, memory for the color of each exemplar was biased towards the central

1251 color (Brady et al., 2018). In Experiment 4, we assigned exemplars of different

1252 manmade objects with colors that determined their typicality. We found that, relative to 1253 atypically colored images, typically colored images were retrieved closer to their 1254 respective cluster, but only when using a measure of unadjusted bias, unlike what was 1255 used in Experiments 1 - 3. It is worth noting that effects observed in Experiment 4 may 1256 be weaker because the object-color associations were new and arbitrary, and thus may 1257 be more difficult to retain relative to the well-known features that determined typicality in 1258 Experiments $1-3$. Regardless, this result suggests that the observed differences in bias 1259 by category typicality is driven in some small part by differences in visual similarity, but 1260 visual similarity is likely one of many features that differentiates typical and atypical 1261 category members and gives rise to differences in their bias.

1262 Another possibility is that because typical category members are the closest 1263 match to their category, they are already closely associated with a rich trove of semantic 1264 knowledge, so there is less of a need to encode other, idiosyncratic details. This may 1265 result in more efficient encoding in circumstances where the information is consistent 1266 with prior knowledge, but may impair in-depth processing of any other details 1267 (Sweegers \& Talamini, 2014; Sweegers et al., 2015). In Experiment 3, we added an 1268 exemplar memory test as a way to understand the depth of encoding, or memory 1269 strength, of typical and atypical category members. Performance on this test depended 1270 on the encoding context: When category information predicted the locations of most 1271 images, memory for atypical category members was greater than memory for typical 1272 ones, but when category information was not relevant during encoding (in the control 
1273 group), there was no difference in exemplar memory by typicality. This suggests that

1274 typical category members may be processed in a way that discounts their idiosyncratic

1275 features when category membership is salient during learning. Alternatively, atypical

1276 images may be more memorable because their distinctiveness from other category

1277 neighbors draws attention to their specific details and preserves their memory as a

1278 result. Consistent with this interpretation, schema-inconsistent memories are sometimes

1279 better remembered than schema-consistent ones (e.g., Bower, Black, \& Turner, 1979;

1280 Sakamoto \& Love, 2004). This suggests that the details of memoranda are better

1281 remembered if they are sufficiently differentiated from the salient organizational

1282 structure guiding learning. This account may also explain the observed differences in

1283 bias when considering exemplar memory accuracy: when exemplar memory was

1284 preserved, typical images were retrieved closer to their category neighbors relative to 1285 atypical images, but when exemplars were forgotten, this difference was no longer 1286 reliable. It may be that for atypical category members, worse exemplar memory is 1287 associated with a larger influence of semantic knowledge, and this larger influence 1288 negates the difference in bias between typical and atypical category members. A caveat 1289 of this interpretation, however, is that the there was no reliable difference in bias for 1290 atypical category members as a function of exemplar memory, as would be predicted by 1291 this account, although the direction of the effect is numerically consistent. Future work 1292 that systematically varies the degree of similarity between memoranda and their 1293 overarching organization could help to better adjudicate between these two accounts.

1294 Interestingly, such a difference in processing the details of the exemplars seems 1295 to be unrelated to processing details of the location. We reported that, in Experiments 2 
1296 and 3, less confident responses were more biased towards category centers, with 1297 typical category members showing greater bias than atypical ones, but confidence in 1298 location memory did not interact with category typicality to influence bias (Figure S3A1299 B). In contrast, images with intact exemplar memory exhibited a robust difference in 1300 bias by typicality, while images whose specific exemplar was forgotten did not. At first 1301 glance, these results seem discrepant, as exemplar memory and location confidence 1302 both seem to index the strength of memory for the specific image-location association, 1303 suggesting both that low-confident responses or incorrect exemplar memory should give 1304 rise more bias due to a stronger influence of semantic knowledge. However, our results 1305 suggest that memory for different elements of an event may differ in their susceptibility 1306 to bias from prior knowledge. Memory for the specific exemplar may be a better index of 1307 the 'semantic' element of each memory, where weaker memory disrupts the biasing 1308 effect of other category members, while location confidence corresponds to an 1309 'episodic' element, which is more prone to distortion by category members if it is weak. 1310 Analogously to prior work showing that episodic memory can be affected by multiple 1311 sources of prior knowledge, like the average size of an object and the average size of 1312 its category members (P. Hemmer \& Steyvers, 2009), it may be that memory of an 1313 event is composed of multiple, separable sources of event-specific information that 1314 differently interact with general or semantic information.

1315 Our priority was to establish whether any aspect of semantic memory could 1316 influence distortions in episodic memory. To achieve this aim, we prioritized developing 1317 a distinction between category members that was robust across participants, choosing 1318 to leverage typicality within categories for the reasons described above. However, 
1319 typicality is one of many ways that category membership is organized and measured.

1320 The graded structure of categories is also well characterized by the frequency that its

1321 members are encountered in the environment; their central tendency, or similarity to

1322 other category members (or, inversely, their distinctiveness from all other category

1323 members); their accessibility, or output dominance, characterized as the frequency with

1324 which an item is produced as an example of a category. These characteristics are often

1325 highly correlated, such that typical category members are more easily accessible

1326 (Schmidt, 1996). Indeed, past observations of clustering in free recall are well explained

1327 by category typicality, but more variance is explained by accessibility (Smith et al.,

1328 2000). Because we did not control for familiarity or accessibility when choosing our

1329 stimuli, it is possible that these dimensions of semantic knowledge could be used to

1330 obtain similar biases in episodic memory. Either way, our results demonstrate that the

1331 internal structure of a category can explain the extent of distortions in new episodic

1332 memories. One promising future avenue of research may to understand potential

1333 commonalities and distinctions in how semantic knowledge distorts new memories

1334 along these multiple dimensions.

\section{Conclusions}

1337 We have presented an investigation of the costs and beneficial influences that semantic 1338 knowledge exerts on new episodic memories. This work demonstrates that semantic 1339 knowledge and episodic memory are closely intertwined and offers an opportunity to 1340 better understand how information supported by these two systems is integrated in the 1341 context of new memory formation. 


\section{Data and Code Availability}

1343 Stimuli, raw data, and analysis code that support the findings of the four memory

1344 experiments will be made publicly accessible upon acceptance of the manuscript. Raw

1345 data and analysis code dedicated to stimulus development will be made available upon

1346 request.

1347

1348 Acknowledgements

1349 This research was funded by NIH F32 NS108511 to A.T. and NIH R01 DC009209 to 1350 S.T.S. 
1352

1353

1354

1355

1356

1357

1358

1359

1360

1361

1362

1363

1364

1365

1366

1367

1368

Addis, D. R., Pan, L., Vu, M.-A., Laiser, N., \& Schacter, D. L. (2009). Constructive episodic simulation of the future and the past: Distinct subsystems of a core brain network mediate imagining and remembering. Neuropsychologia, 47(11), 22222238.

Anderson, J. R. (1983). A spreading activation theory of memory. Journal of Verbal Learning and Verbal Behavior, 22(3), 261-295.

Anderson, J. R., \& Ross, B. H. (1980). Evidence against a semantic-episodic distinction. Journal of Experimental Psychology: Human Learning and Memory, 6(5), 441466.

Bartlett, F. C. (1920). Some experiments on the reproduction of folk-stories. Folklore, 31(1), 30-47.

Bartlett, F. C. (1932). A theory of remembering. In Remembering: A study in experimental and social psychology. New York: Cambridge University Press.

Bein, O., Reggev, N., \& Maril, A. (2014). Prior knowledge influences on hippocampus and medial prefrontal cortex interactions in subsequent memory. Neuropsychologia, 64, 320-330.

Bejjani, C., \& Egner, T. (2019). Spontaneous task structure formation results in a cost to incidental memory of task stimuli. Frontiers in Psychology, 10.

Bousfield, W. A., Cohen, B. H., \& Whitmarsh, G. A. (1958). Associative clustering in the recall of words of different taxonomic frequencies of occurrence. Psychological Reports, 4, 39-44.

Bower, G. H., Black, J. B., \& Turner, T. J. (1979). Scripts in memory for text. Cognitive Psychology, 11(2), 177-220.

Bozeat, S., Ralph, M. A. L., Graham, K. S., Patterson, K., Wilkin, H., Rowland, J., ... Hodges, J. R. (2003). A duck with four legs: Investigating the structure of 
conceptual knowledge using picture drawing in semantic dementia. Cognitive Neuropsychology, 20(1), 27-47.

Brady, T. F., Schacter, D. L., \& Alvarez, G. A. (2018). The adaptive nature of false memories is revealed by gist- based distortion of true memories. PsyArXiv.

Brainard, D. H., Longère, P., Delahunt, P. B., Freeman, W. T., Kraft, J. M., \& Xiao, B. (2006). Bayesian model of human color constancy. Journal of Vision, 6(11), 10 10.

Bransford, J. D., \& Johnson, M. K. (1972). Contextual prerequisites for understanding: Some investigations of comprehension and recall. Journal of Verbal Learning and Verbal Behavior, 11(6), 717-726.

Brodeur, M. B., Dionne-Dostie, E., Montreuil, T., \& Lepage, M. (2010). The Bank of Standardized Stimuli (BOSS), a new set of 480 normative photos of objects to be used as visual stimuli in cognitive research. PLOS ONE, 5(5), e10773.

Carey, S., \& Bartlett, E. (1978). Acquiring a single new word. Papers and Reports on Child Language Development, 15, 17-29.

Collins, A. M., \& Loftus, E. F. (1975). A spreading-activation theory of semantic processing. Psychological Review, 82(6), 407-428.

Collins, A., \& Quillian, R. (1969). Retrieval time from semantic memory. Journal of Verbal Learning and Verbal Behavior, 8, 240-247.

Connolly, A. C., Gleitman, L. R., \& Thompson-Schill, S. L. (2007). Effect of congenital blindness on the semantic representation of some everyday concepts. Proceedings of the National Academy of Sciences, 104(20), 8241-8246.

Coutanche, M. N., \& Koch, G. E. (2017). Variation across individuals and items determine learning outcomes from fast mapping. Neuropsychologia, 106(Supplement C), 187-193. 
1402

1403

1404

1405

1406

1407

1408

1409

1410

1411

1412

1413

1414

1415

1416

1417

1418

1419

1420

1421

1422

1423

1424

1425

Coutanche, M. N., \& Thompson-Schill, S. L. (2014). Fast mapping rapidly integrates information into existing memory networks. Journal of Experimental Psychology: General, 143(6), 2296-2303.

Cox, G. E., Hemmer, P., Aue, W. R., \& Criss, A. H. (2018). Information and processes underlying semantic and episodic memory across tasks, items, and individuals. Journal of Experimental Psychology: General, 147(4), 545-590.

Craik, F. I. M., \& Lockhart, R. S. (1972). Levels of processing: A framework for memory research. Journal of Verbal Learning and Verbal Behavior, 11(6), 671-684.

Craik, F. I. M., \& Tulving, E. (1975). Depth of processing and the retention of words in episodic memory. Journal of Experimental Psychology: General, 104(3), 268294.

Deyne, S. D., Verheyen, S., Ameel, E., Vanpaemel, W., Dry, M. J., Voorspoels, W., \& Storms, G. (2008). Exemplar by feature applicability matrices and other Dutch normative data for semantic concepts. Behavior Research Methods, 40(4), 1030-1048.

Dunsmoor, J. E., \& Murphy, G. L. (2014). Stimulus typicality determines how broadly fear is generalized. Psychological Science, 25(9), 1816-1821.

Ghosh, V. E., \& Gilboa, A. (2014). What is a memory schema? A historical perspective on current neuroscience literature. Neuropsychologia, 53, 104-114.

Goodman, G. S. (1980). Picture memory: How the action schema affects retention. Cognitive Psychology, 12(4), 473-495.

Goulet-Pelletier, J.-C., \& Cousineau, D. (2018). A review of effect sizes and their confidence intervals, Part I: The Cohen's d family. The Quantitative Methods for Psychology, 14(4), 242-265. 
Greenberg, D. L., \& Verfaellie, M. (2010). Interdependence of episodic and semantic memory: Evidence from neuropsychology. Journal of the International Neuropsychological Society, 16(5), 748-753.

Hemmer, P., \& Steyvers, M. (2009). Integrating episodic memories and prior knowledge at multiple levels of abstraction. Psychonomic Bulletin \& Review, 16(1), 80-87.

Hemmer, Pernille, \& Persaud, K. (2014). Interaction between categorical knowledge and episodic memory across domains. Frontiers in Psychology, 5.

Heussen, D., Poirier, M., Hampton, J. A., \& Aldrovandi, S. (2011). An effect of semantic memory on immediate memory in the visual domain. In B. Kokinov, A. KarmiloffSmith, \& N. J. Nersessian (Eds.), European Perspectives on Cognitive Science (p. 6). New Bulgarian University Press.

Howard, M. W., \& Kahana, M. J. (2002). When Does Semantic Similarity Help Episodic Retrieval? Journal of Memory and Language, 46(1), 85-98.

Huttenlocher, J., Hedges, L. V., \& Duncan, S. (1991). Categories and particulars: Prototype effects in estimating spatial location. Psychological Review, 98(3), 352.

Huttenlocher, J., Hedges, L. V., \& Vevea, J. L. (2000). Why do categories affect stimulus judgment? Journal of Experimental Psychology: General, 129(2), 220241.

Kirwan, C. B., Jones, C. K., Miller, M. I., \& Stark, C. E. L. (2007). High-resolution fMRI investigation of the medial temporal lobe. Human Brain Mapping, 28(10), 959966.

Knill, D. C., \& Richards, W. (Eds.). (1996). Perception as Bayesian inference. Cambridge University Press.

Konkle, T., \& Caramazza, A. (2013). Tripartite organization of the ventral stream by animacy and object size. Journal of Neuroscience, 33(25), 10235-10242. 
1451 Koutstaal, W., \& Schacter, D. L. (1997). Gist-based false recognition of pictures in older and younger adults. Journal of Memory and Language, 37(4), 555-583.

1453 Lakens, D. (2013). Calculating and reporting effect sizes to facilitate cumulative science: 1454 a practical primer for t-tests and ANOVAs. Frontiers in Psychology, 4.

1455 Lewis, P. A., \& Durrant, S. J. (2011). Overlapping memory replay during sleep builds 1456 cognitive schemata. Trends in Cognitive Sciences, 15(8), 343-351.

1457 Loftus, E. F., \& Palmer, J. C. (1974). Reconstruction of automobile destruction: An 1458 example of the interaction between language and memory. Journal of Verbal Learning and Verbal Behavior, 13(5), 585-589.

Love, B. C., Medin, D. L., \& Gureckis, T. M. (2004). SUSTAIN: A network model of category learning. Psychological Review, 111(2), 309-332.

McClelland, J. L., McNaughton, B. L., \& O'Reilly, R. C. (1995). Why there are complementary learning systems in the hippocampus and neocortex: Insights from the successes and failures of connectionist models of learning and memory. Psychological Review, 102(3), 419-457.

McKoon, G., \& Ratcliff, R. (1979). Priming in episodic and semantic memory. Journal of Verbal Learning and Verbal Behavior, 18, 463-480.

McKoon, G., Ratcliff, R., \& Dell, G. S. (1986). A critical evaluation of the semanticepisodic distinction, 12(2), 295-306.

1470 Osherson, D. N., Smith, E. E., Wilkie, O., López, A., \& Shafir, E. (1990). Categorybased induction. Psychological Review, 97(2), 185-200.

1472 Patterson, K. (2007). The reign of typicality in semantic memory. Philosophical Transactions of the Royal Society B: Biological Sciences, 362(1481), 813-821.

1474 Payne, J. D., Tucker, M. A., Ellenbogen, J. M., Wamsley, E. J., Walker, M. P., Schacter, 1475 D. L., \& Stickgold, R. (2012). Memory for semantically related and unrelated 
declarative information: The benefit of sleep, the cost of wake. PLoS ONE, 7(3), e33079.

Persaud, K., \& Hemmer, P. (2016). The dynamics of fidelity over the time course of long-term memory. Cognitive Psychology, 88, 1-21.

Posner, M. I., Goldsmith, R., \& Welton Jr, K. E. (1967). Perceived distance and the classification of distorted patterns. Journal of Experimental Psychology, 73(1), 28.

Posner, M. I., \& Keele, S. W. (1968). On the genesis of abstract ideas. Journal of Experimental Psychology, 77(3p1), 353.

Ratcliff, R., \& McKoon, G. (1995). Bias in the priming of object decisions. Journal of Experimental Psychology: Learning, Memory, and Cognition, 21(3), 754-767.

Rips, L. J. (1975). Inductive judgments about natural categories. Journal of Verbal Learning and Verbal Behavior, 14(6), 665-681.

Roediger, H. L., \& McDermott, K. B. (1995). Creating false memories: Remembering words not presented in lists. Journal of Experimental Psychology: Learning, Memory, and Cognition, 21(4), 803.

Rogers, T. T., Patterson, K., Jefferies, E., \& Lambon Ralph, M. A. (2015). Disorders of representation and control in semantic cognition: Effects of familiarity, typicality, and specificity. Neuropsychologia, 76, 220-239.

Rosch, E. H. (1973). On the internal structure of perceptual and semantic categories. In T. E. Moore (Ed.), Cognitive development and acquisition of language (pp. 111144). San Diego: Academic Press.

Rosch, E., \& Mervis, C. B. (1975). Family resemblances: Studies in the internal structure of categories. Cognitive Psychology, 7(4), 573-605. 
1500 Rosch, E., Mervis, C. B., Gray, W. D., Johnson, D. M., \& Boyes-Braem, P. (1976). Basic objects in natural categories. Cognitive Psychology, 8(3), 382-439.

Russo, N., Hagmann, C. E., Andrews, R., Black, C., Silberman, M., \& Shea, N. (2018). Validation of the C.A.R.E. stimulus set of 640 animal pictures: Name agreement and quality ratings. PLOS ONE, 13(2), e0192906.

Sakamoto, Y., \& Love, B. C. (2004). Schematic influences on category learning and recognition memory. Journal of Experimental Psychology: General, 133(4), 534553.

Schacter, D. L., \& Addis, D. R. (2007). The cognitive neuroscience of constructive memory: remembering the past and imagining the future. Philosophical Transactions of the Royal Society of London B: Biological Sciences, 362(1481),

Schmidt, S. R. (1996). Category typicality effects in episodic memory: Testing models of distinctiveness. Memory \& Cognition, 24(5), 595-607.

Schulman, A. I. (1974). Memory for words recently classified. Memory \& Cognition, 2(1), $47-52$.

Simons, J. S., Graham, K. S., \& Hodges, J. R. (2002). Perceptual and semantic contributions to episodic memory: evidence from semantic dementia and Alzheimer's disease. Journal of Memory and Language, 47(2), 197-213.

1519 Smith, S. M., Ward, T. B., Tindell, D. R., Sifonis, C. M., \& Wilkenfeld, M. J. (2000). Category structure and created memories. Memory \& Cognition, 28(3), 386-395.

1521 Stark, S. M., Yassa, M. A., Lacy, J. W., \& Stark, C. E. L. (2013). A task to assess behavioral pattern separation (BPS) in humans: Data from healthy aging and mild cognitive impairment. Neuropsychologia, 51(12), 2442-2449. 
Sweegers, C. C. G., Coleman, G. A., van Poppel, E. a. M., Cox, R., \& Talamini, L. M. (2015). Mental schemas hamper memory storage of goal-irrelevant information. Frontiers in Human Neuroscience, 9, Article 629.

Sweegers, C. C. G., \& Talamini, L. M. (2014). Generalization from episodic memories across time: A route for semantic knowledge acquisition. Cortex, 59, 49-61.

Tse, D., Takeuchi, T., Kakeyama, M., Kajii, Y., Okuno, H., Tohyama, C., ... Morris, R. G. M. (2011). Schema-dependent gene activation and memory encoding in neocortex. Science, 333(6044), 891-895.

Tulving, E. (1972). Episodic and semantic memory. In E. Tulving \& W. Donaldson (Eds.), Organization of Memory. New York: Academic Press.

Uyeda, K. M., \& Mandler, G. (1980). Prototypicality norms for 28 semantic categories. Behavior Research Methods \& Instrumentation, 12(6), 587-595.

van Kesteren, M. T. R., Rijpkema, M., Ruiter, D. J., \& Fernández, G. (2013). Consolidation differentially modulates schema effects on memory for items and associations. PLoS ONE, 8(2), e56155.

van Kesteren, M. T. R. van, Fernández, G., Norris, D. G., \& Hermans, E. J. (2010). Persistent schema-dependent hippocampal-neocortical connectivity during memory encoding and postencoding rest in humans. Proceedings of the National Academy of Sciences, 107(16), 7550-7555.

van Kesteren, M. T. R. van, Rijpkema, M., Ruiter, D. J., \& Fernández, G. (2010). Retrieval of associative information congruent with prior knowledge is related to increased medial prefrontal activity and connectivity. The Journal of Neuroscience, 30(47), 15888-15894.

Webb, C. E., Turney, I. C., \& Dennis, N. A. (2016). What's the gist? The influence of schemas on the neural correlates underlying true and false memories. Neuropsychologia, 93, Part A, 61-75. 


\section{Supplemental Results}

All Experiments

3 Error by typicality: While we had strong predictions that category typicality would

4 influence images' direction of error, it was less clear if category typicality would similarly

5 affect their magnitude of error. For example, the use of category knowledge may have

6 created small, systematic distortions for typical category members, but atypical category

7 members may have exhibited the same extent of error in all directions. Here, we plot

8 average error by typicality in the four experiments (Figure S1). We found that in

9 Experiments $1-3$, error was greater for typical category members relative to atypical

10 category members in the experimental group, suggesting that the influence of category

11 knowledge resulted in greater error in addition to systematic bias. Statistics are reported

12 in the main text for Experiments 1, 2 and 4, and in the supplement for Experiment 3.

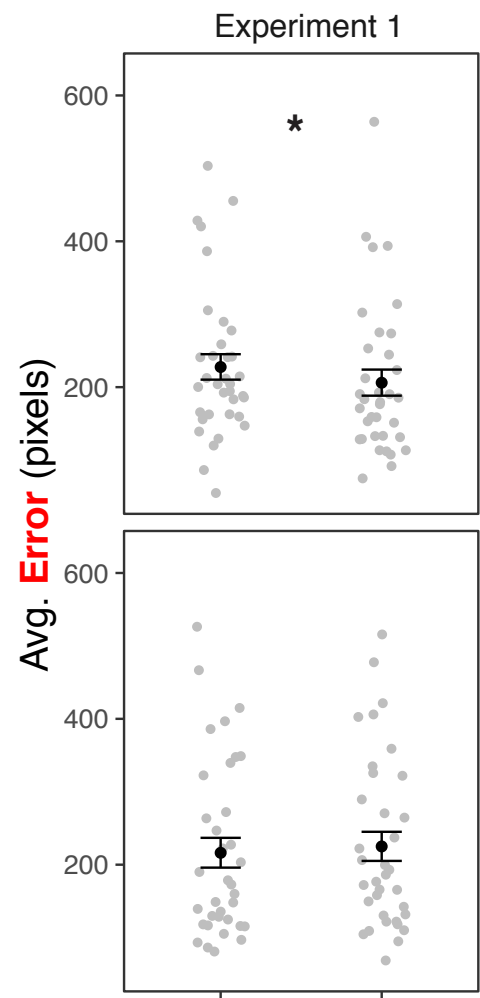

Typical Atypical

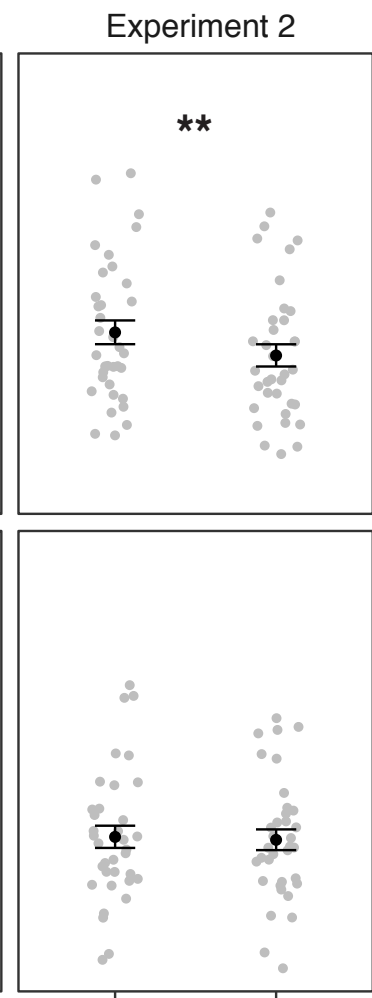

Typical Atypical
Experiment 3
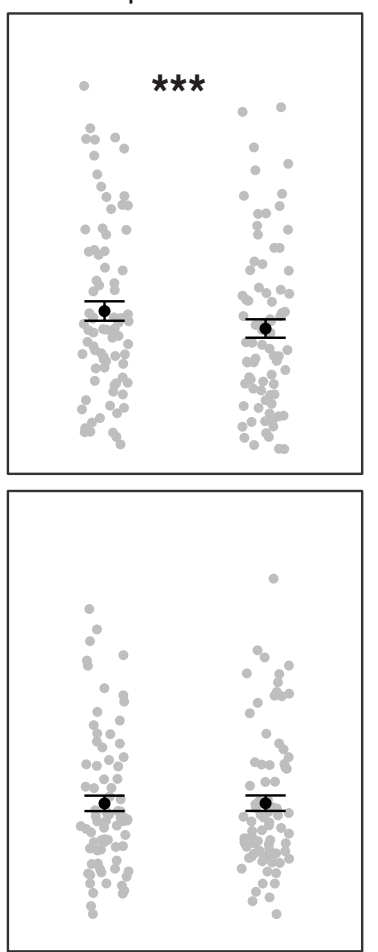

Typical Atypical
Experiment 4
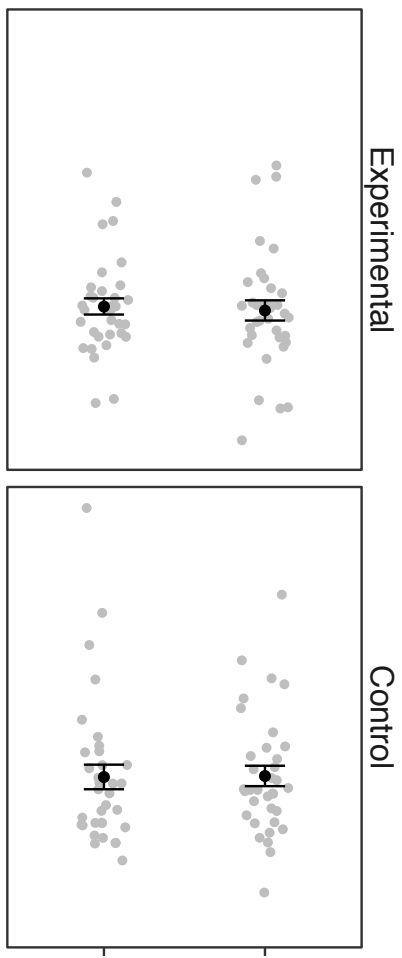

Typical Atypical

Figure S1. Average memory accuracy by category typicality in each experiment. Gray dots signify participants. Error bars indicate SEM. ${ }^{*} p<.05 ;{ }^{* *} p<.01 ;{ }^{* *} p<.001$ 
17 Error by spatial consistency and confidence: In the main text, we reported how 18 confidence and spatial consistency with category membership interacted to influence error in Experiment 2, finding that the difference in error by spatial consistency was greatest when reported confidence was weakest. We aimed to replicate this effect in

21 Experiments 3 and 4 . See Figure S4 for a comparison of the effect sizes across the 22 three experiments.

In Experiment 3, focusing on the experimental group, we entered spatial consistency, confidence, and their interaction into a mixed-effect model with error as the dependent measure (Figure S2B). This revealed main effects of consistency, $F_{(1,149.87)}=$ 81.47, $p<.001$, and confidence, $F_{(2,129.49)}=99.17, p<.001$, qualified by an interaction, $F_{(2,1625.85)}=3.32, p=.04$. This interaction was driven by the same pattern of effects as Experiment 1, with less of a difference in error between spatially consistent and inconsistent images for increasingly confident responses (very confident: $t_{(708.66)}=-2.63$, $p=.009$; somewhat confident: $t_{(325.63)}=-7.14, p<.001$; guessed: $t_{(303.20)}=-7.77, p<$ .001 ; Bonferroni-corrected $\alpha=.017$ for 3 tests). This replicates the effects observed in Experiment 2 and suggests that when memory for specific image locations was weaker, participants relied more on their knowledge of a category's likely location on the grid.

In Experiment 4, using the same model (Figure S2C), we found main effects of spatial consistency, $F_{(1,50.52)}=49.08, p<.001$, and confidence, $F_{(3,39.35)}=8.63, p<$ .001 , but no consistency by confidence interaction, $F_{(3,1129.60)}=1.77, p=.15$. For consistency with Experiments 2 and 3, we computed pairwise tests of error by consistency for each level of reported confidence. This revealed less error for spatially consistent images relative to inconsistent images at all levels of confidence (all $t<-2.84$, all $p<.005$; Bonferroni-corrected $\alpha=.0125$ for 4 tests). The absence of an interaction in Experiment 4 may be driven by the change in stimuli for this experiment, which may 42 have changed the way participants used the confidence responses. For example, visual 43 inspection suggests that the main effect of confidence is much stronger in Experiment 2 44 and 3 relative to Experiment 4, as the spread of error across levels of confidence is 45 much more narrow in Experiment 4, possibly signifying that confidence is a less 46 informative marker of memory strength in this experiment. 
A

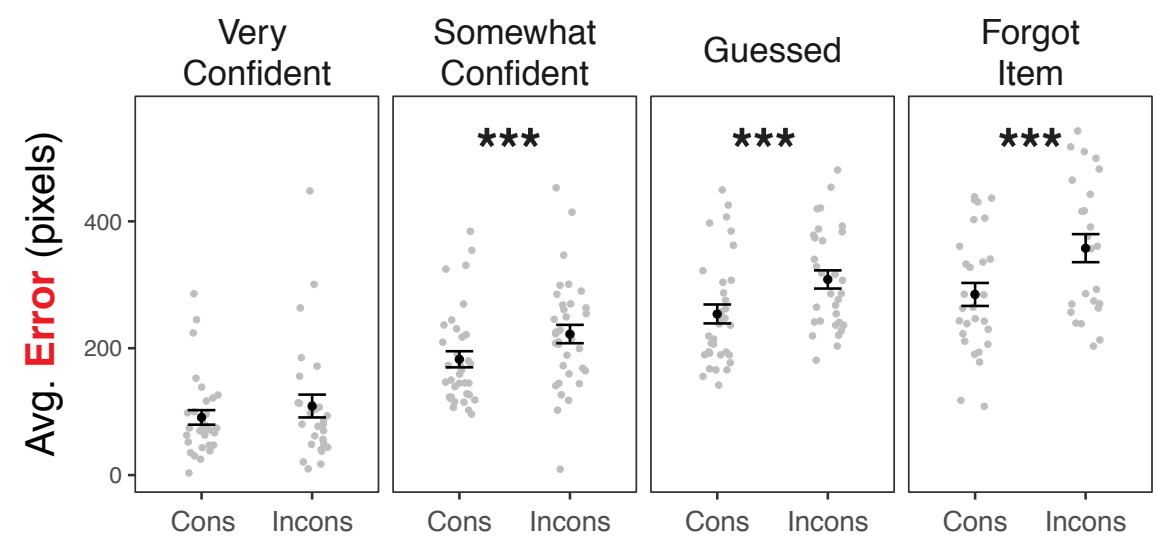

B

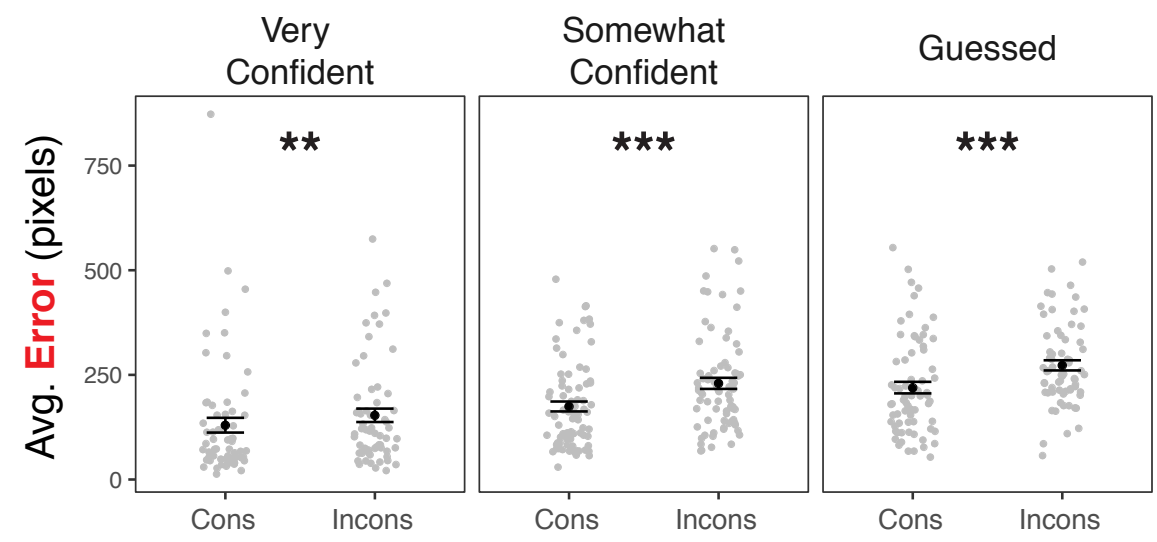

C

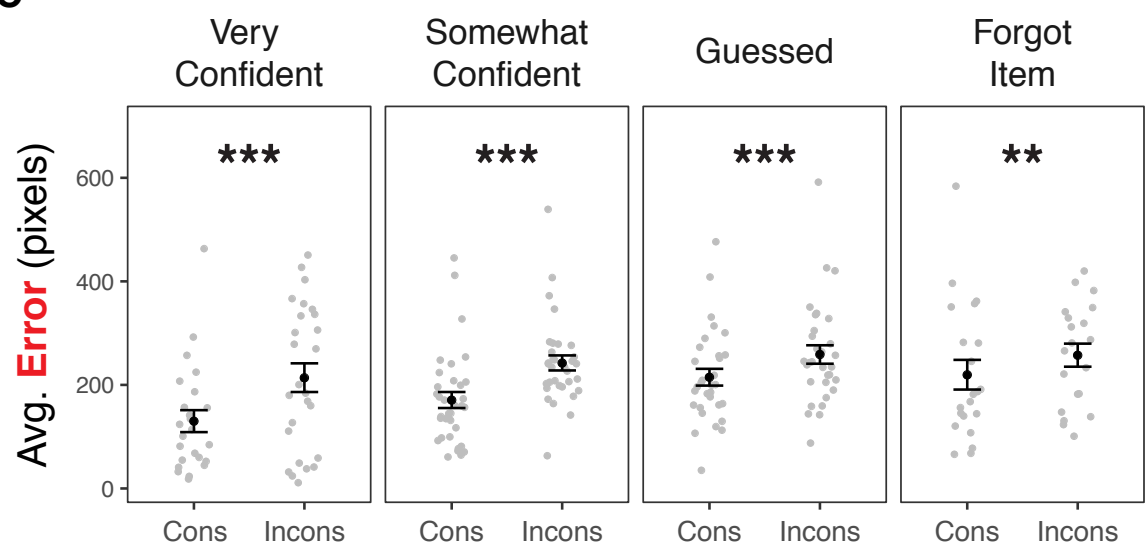

Figure S2. Error by consistency and confidence. Average error by confidence and consistency in Experiment 2 (A), Experiment 3 (B), and Experiment 4 (C). The data from Experiment 2 (replotted from Figure 3D) is included above for comparison with the other experiments. Gray dots signify participants. Error bars indicate SEM. Statistics reflect simple effects that survive Bonferroni correction for the number of tests conducted within each experiment (Experiments $2 \& 4: \alpha=.0125$, Experiment 3: $\alpha=.0167$ ). ${ }^{* *} p<.01 ;{ }^{* * *} p<.001$ 
54 Bias by typicality and confidence: We also sought to replicate the influence of 55 confidence on bias observed in Experiment 2, which revealed a main effect of confidence such that there was less bias for the most confident responses. See Figure S4 for a comparison of the effect sizes across the three experiments.

In Experiments 3 and 4, we computed separate mixed effects models with confidence and typicality with bias as the dependent variable, again focusing only on the experimental group. In Experiment 3 (Figure S3B), this model revealed a main effect of confidence, $F_{(2,98.64)}=3.80, p=.03$, and a main effect of category typicality, $F_{(1,}$ $1921.76)=21.85, p<.001$. There was no reliable interaction, $F_{(2,2908.71)}=2.21, p=.11$. As in Experiment 2, the main effect of confidence was driven by less bias for 'very confident' responses relative to 'somewhat confident' responses, $t_{(78.79)}=-2.47, \mathrm{p}=$ .016 , and relative to 'guessed' responses, $t_{(101.64)}=-2.52, p=.013$, both of which survive correction for multiple corrections ( 3 tests, $\alpha=.0167$ ). There was no difference in bias between 'somewhat confident' and 'guessed' responses, $t_{(91.66)}=-0.67, p=0.50$. The main effect of typicality was driven by greater bias for typical category members relative to atypical category members for 'very confident' responses, $t_{(622.79)}=3.27, p=$ .001 , and for 'somewhat confident' responses, $t_{(329.80)}=3.56, p<.001$, but not for 'guessed' responses, $t_{(279.10)}=1.18, p=.24$ (3 tests, $\left.\alpha=0.167\right)$. In Experiment 4 (Figure $\mathrm{S} 3 \mathrm{C}$ ), the same group $\mathrm{x}$ typicality ANOVA revealed no main effects or 73 interaction, all $F<1.85$, all $p>.15$.

Retrieval in Experiments 2 and 3 was modulated by reported confidence such that stronger memories, as indexed by high confidence, were less biased towards their category centers. This suggests that retrieval of weaker memories relied more on knowledge of each category's general location and thus memory for their locations was more distorted. Furthermore, the main effect of category typicality in Experiment 3 suggests that confidence and category typicality may separately and independently bias

80 retrieval, although this was not observed in Experiment 2. Interestingly, in Experiment 4, 81 bias was not affected by confidence at all, despite other observations that more 82 confidence was related to memory accuracy. This null result may be explained by the 83 possibility that confidence was not as strong as an index of memory as it was in 84 Experiments $1-3$. 
A

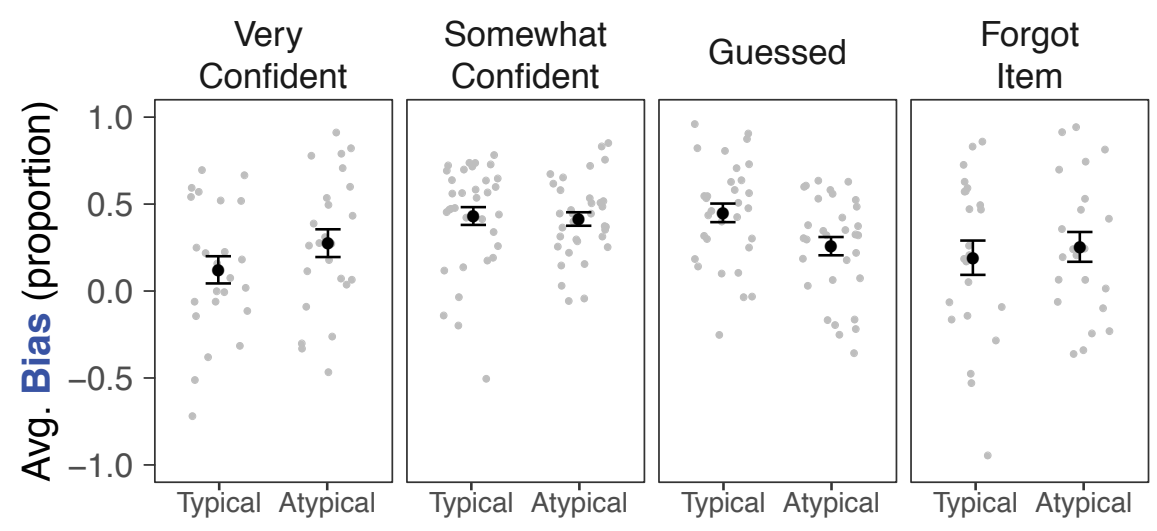

B

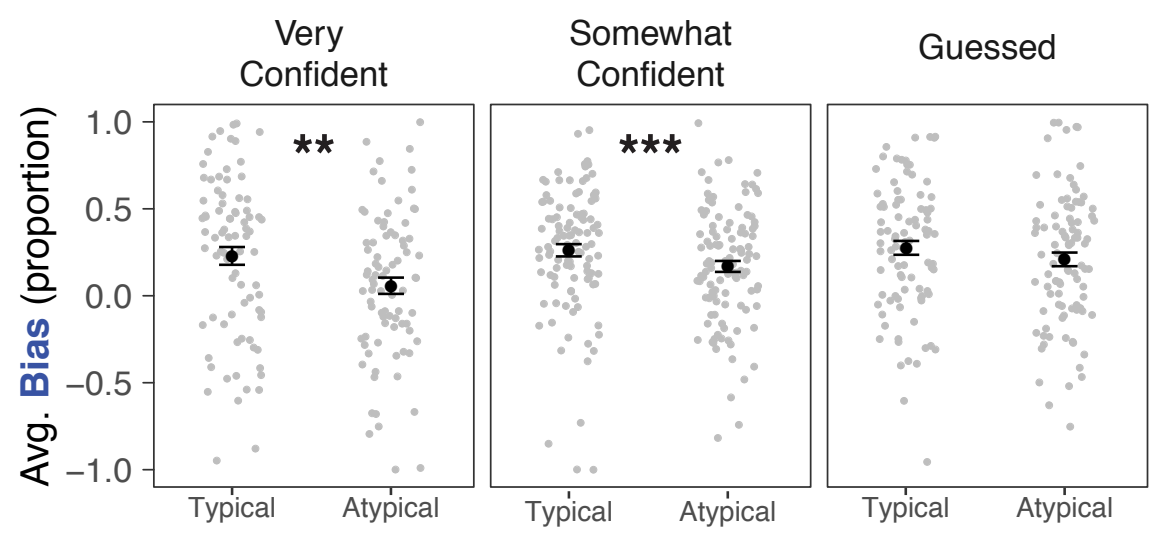

C

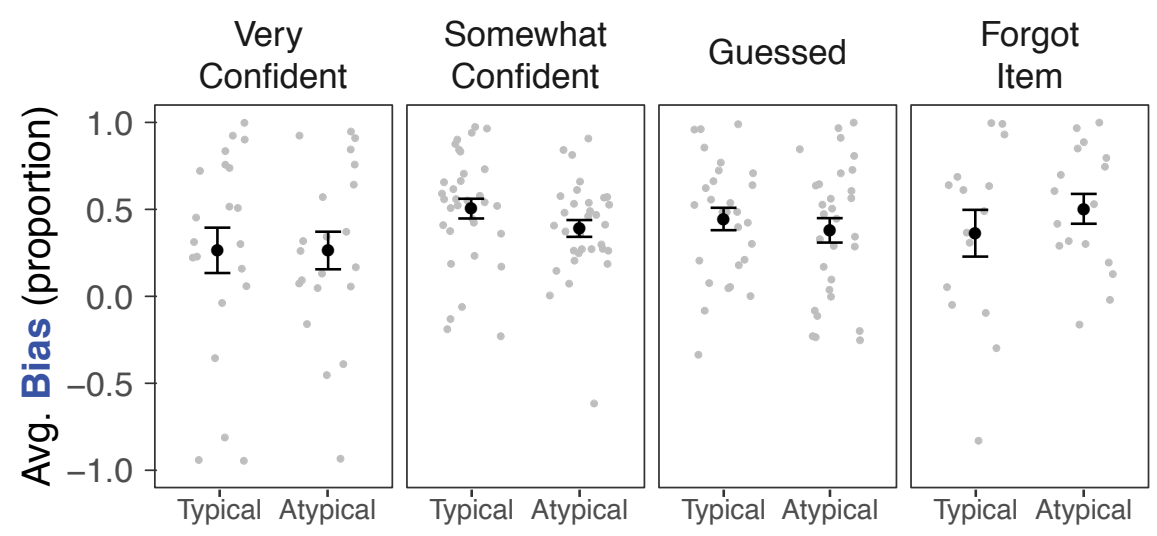

Figure S3. Bias by typicality and confidence. Average bias (as a proportion of error) by confidence and typicality in Experiment 2 (A), Experiment 3 (B), and Experiment 4 (C). Results from Experiment 2 (replotted from Figure 3B) are included here for comparison with other experiments. Gray dots signify participants. Error bars indicate SEM. Statistics reflect simple effects that survive Bonferroni correction for the number of tests conducted within each experiment (Experiments $2 \& 4: \alpha=.0125$, Experiment 3: $\alpha=.0167$ ). ${ }^{* *} p<.01 ;{ }^{* * *} p<.001$ 
SDM: Error

Consistent - Inconsistent

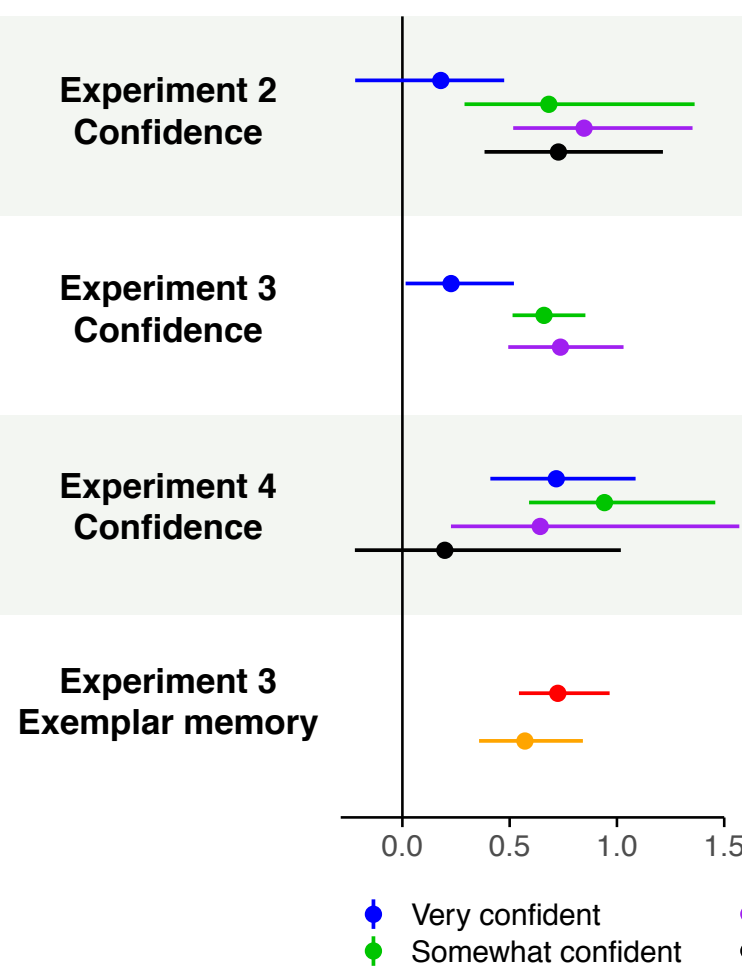

SDM: Proportion bias

Averaged across category typicality

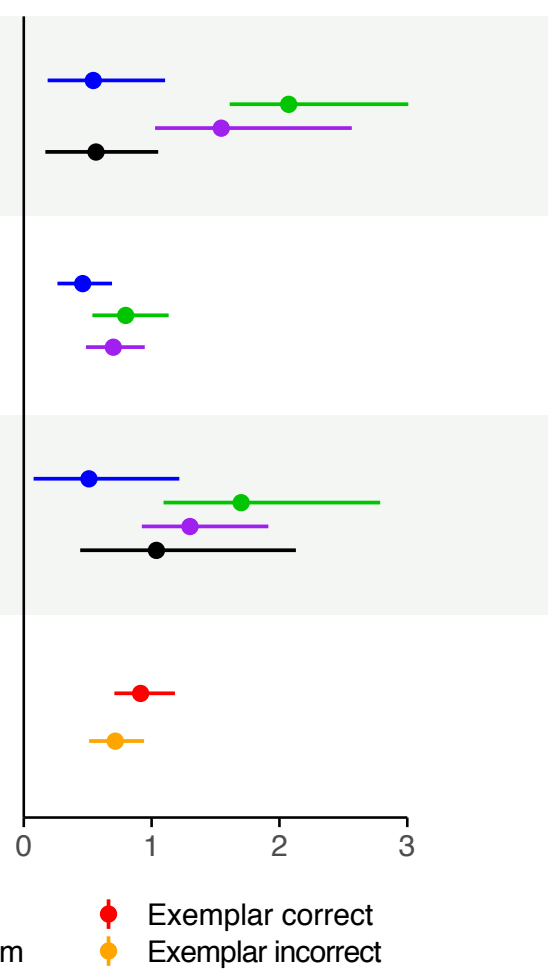

Figure S4. Effect sizes of error and bias across experiments, by confidence and exemplar 94 memory. (A) Standardized difference in mean error for spatially consistent versus inconsistent 95 images. 0 on the $x$-axis reflects no difference in error by consistency. (B) Standardized 96 difference in the mean bias relative to 0 , averaged across typical and atypical images. 0 on the $97 \mathrm{x}$-axis reflects no reliable bias towards category clusters. Points represent mean effect size. 98 Lines represent bootstrapped 95\% confidence intervals. 
Swap errors: One open question is whether the observed distortions in memory are due to small but systematic changes in retrieval, or large errors where participants mistook an image for a different one - a swap error. Although there is no clear way to identify swaps in these experiments, one way to adjudicate between these possibilities is by approximating the profile of a swap error by identifying retrieved locations that were closer to their category cluster. Since most members of a category were clustered in the same area, a swap error would look like a very large bias towards its category cluster. While this profile cannot directly pinpoint swap errors, decomposing retrieval in this way may provide a course hint at possible differences in biases.

To quantify this, we drew a line between each image's encoded location and cluster center, and we projected its retrieved location onto the line. We then sorted the projected distances of all images into four bins, separated by three landmarks along the line: the image's encoded location, its category's cluster center, and the halfway point between the two (Figure S5). The resulting four bins were: (1) 'Biased away', or images whose projected retrieval was father from their cluster center relative to their encoded locations, (2) 'Closer to Encoded', or images whose retrieval was biased towards their cluster center, but was retrieved closer to its encoded location than its cluster center, (3) 'Closer to Cluster', or images whose retrieval was closer to their cluster center than their encoded location, and (4) 'Beyond Cluster', for images retrieved in locations extending beyond their cluster center. The proportions of trials in these bins were entered into ANOVAs separately for each experiment. Planned paired t-tests of the difference by typicality were performed separately for each bin. Significance for these tests was Bonferroni-corrected for four comparisons $(\alpha=.0125)$. main effect of bin, $F_{(3,102)}=15.93, p<.001, \eta_{p}^{2}=0.50$, a main effect of typicality, $F_{(1,34)}$

125 This interaction was driven by more atypical category members biased away from their cluster center relative to typical ones $p, t_{(34)}=-3.04, p=.004, d=-0.51$. No other 127 comparisons reached significance, $p>0.09$.

In Experiment 2, the same ANOVA revealed similar effects: a main effect of bin, $F_{(3,102)}=37.12, p<.001, \eta_{p}{ }^{2}=0.77$, no reliable main effect of typicality, $F_{(1,34)}=1.03, p$ 

$=.32, \eta_{p}^{2}=0.00$, and a reliable interaction, $F_{(3,102)}=10.54, p<.001, \eta_{p}^{2}=0.24$. Here, 131 there were more atypical trials than typical trials that were biased away from their 132 cluster center, $t_{(34)}=-3.65, p<.001, d=-0.62$, and biased towards the cluster center 133 but still retrieved closer to their encoded location, $t_{(34)}=-3.05, p=.004, d=-0.52$. 134 However, more typical trials were retrieved closer to their cluster center, $t_{(34)}=3.27, p=$ $.002, d=0.55$, and beyond their cluster center, $t_{(34)}=3.40, p=.002, d=0.58$. In Experiment 3, we again found a main effect of bin, $F_{(3,342)}=35.62, p<.001$, $\eta_{p}^{2}=0.43$, no reliable main effect of typicality, $F_{(1,114)}=0.27, p=.61, \eta_{p}^{2}=0.00$, and a reliable interaction, $F_{(3,342)}=5.21, p=.002, \eta_{p}{ }^{2}=0.04$. Here, more atypical trials were biased away from their cluster center, $t_{(114)}=-3.82, p<.001, d=-0.36$, and more typical trials were retrieved beyond their cluster center $t_{(114)}=3.11, p=.002, d=0.29$. No other comparisons reached significance, all $p>0.28$.

We found a different pattern in Experiment 4. The same ANOVA revealed a main effect of bin, $F_{(3,102)}=15.59, \mathrm{p}<.001, \eta_{\mathrm{p}}^{2}=0.54$, no main effect of typicality, $F_{(1,34)}=$ $0.07, p=.80, \eta_{p}^{2}=0.00$, and no interaction, $F_{(3,102)}=0.22, p=.88, \eta_{p}^{2}=0.006$. Unlike in Experiments $1-3$, more trials were retrieved closer to their cluster center than to their encoded locations.

Taken together, these results suggest that a mixture of different errors drives the observed biases in retrieval. It seems likely that many of participants' memories for both typical and atypical category members can be characterized as smaller distortions rather than larger swap errors, as the plurality of trials were biased towards their cluster center but still remained closer to their encoded locations. However, the large proportion of trials retrieved closer to or beyond their category cluster also highlights the possibility 153 that swap errors were also common. When considering typicality, we find that typical category members are more likely to be retrieved closer to or beyond their cluster center relative to atypical ones in Experiments 2 and 3 (and in the same direction in 156 Experiment 1), suggesting that swap errors may contribute more to retrieval of typical 157 category members relative to atypical category members. Finally, memory in 158 Experiment 4 may have been more prone to swap errors, likely due to visually similar 159 exemplars of the six objects that were encoded, rather than visually distinct members of 160 six categories. 


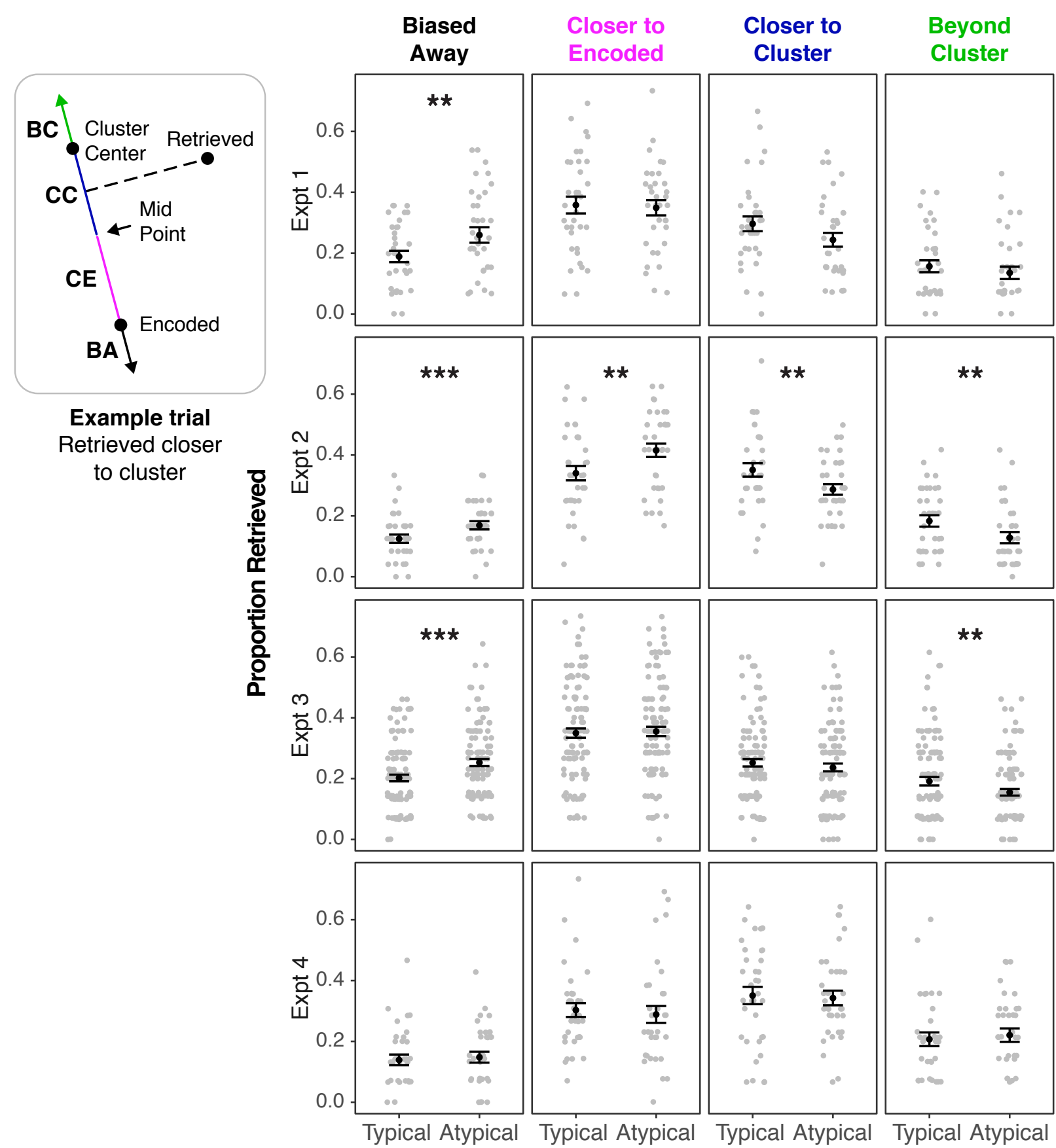

Figure S5. Swap error analyses. Proportion of images retrieved, relative to encoded locations and cluster centers. 'Biased Away' indicates the proportion of trials retrieved farther from the cluster center than what was encoded. 'Closer to Encoded' indicates the proportion of retrieved closer to their encoded location than to their cluster center. 'Closer to Cluster' indicates the proportion retrieved closer to their cluster center than their encoded locations. 'Beyond Cluster' indicates the proportion retrieved beyond their category cluster relative to their encoded location. Gray dots signify participants. Error bars indicate SEM. Statistics reflect two-tailed paired-sample t-tests ( $\alpha=.0125$. Bonferroni-corrected for 4 comparisons per experiment). ${ }^{* *} p<$ $.01^{* * *} p<.001$ 


\section{Experiment 3}

172 Exemplar memory by typicality and confidence: We used participants' reports of 173 confidence about their chosen images in the exemplar memory test to gain confidence

174 in this measure as an indication of memory strength. To do so, we computed a mixed175 effects logistic regression with exemplar memory as a binary dependent variable and 176 confidence, typicality, and their interaction as independent variables. These analyses 177 revealed a main effect of confidence, $X^{2}(2)=97.73, p<.001$, with a greater likelihood of 178 correct exemplar memory for images with higher confidence ratings. There was also a 179 main effect of typicality, $X^{2}(1)=4.75, p=.03$, with a greater likelihood of correct 180 exemplar memory for atypical images relative to typical images, as was reported in the 181 main text. There was no reliable interaction between confidence and typicality, $X^{2}(2)=$ $1821.95, p=.38$. Overall, participants' confidence ratings reflected the likelihood that they 183 chose the image that they had encoded, providing justification for our decision to use 184 exemplar memory as an index of memory for the perceptual details of the images.

Error by spatial consistency with semantic knowledge: Before considering location memory as a function of exemplar memory, we first sought to replicate the observations 188 from Experiments 1 and 2 that memory was more accurate for images that were spatially consistent with their category membership. We computed a 2 (group: experimental, control) x 2 (spatial consistency: consistent, consistent) ANOVA. This revealed a main effect of spatial consistency, $F_{(1,228)}=96.14, p<.001, \eta_{p}^{2}=0.30$, and no main effect of group, $F_{(1,228)}=0.007, p=.93, \eta_{p}^{2}=0.001$, qualified by an interaction, $F_{(1,228)}=28.48, p<.001, \eta_{p}^{2}=0.11$. Both groups exhibited greater error for spatially 194 inconsistent images relative to inconsistent images (experimental: $t_{(114)}=-10.47, p<$ $195.001, d=-0.98$; control: $\left.t_{(114)}=-3.23, p=.002, d=-0.30\right)$, although the interaction 196 indicated that the effect was reliably stronger in the experimental group. As in 197 Experiment 4, the dense clustering of images in spatially consistent locations may have aided retrieval in both groups. However, the reliably stronger effect in the experimental group demonstrates that retrieval was more accurate for images that were spatially consistent with their category membership, replicating Experiments 1 and 2. 
202 Bias by category typicality: Before considering how exemplar memory related to bias in 203 location memory, we first aimed to replicate the finding that typical category members 204 were retrieved closer to their category's cluster relative to atypical members, as was 205 observed in Experiments 1 and 2. We computed a 2 (group: experimental, control) $\times 2$ 206 (typicality: typical, atypical) ANOVA amongst spatially inconsistent images, with the 207 average proportion of bias as the dependent variable. This revealed no main effect of 208 group, $F_{(1,228)}=0.002, p=.96, \eta_{p}^{2}=0.00$, but a main effect of typicality, $F_{(1,228)}=13.76$, $209 p<.001, n_{p}^{2}=0.06$, qualified by a reliable interaction, $F_{(1,228)}=10.53, p=.001, n_{p}^{2}=$ 210 0.04. As in Experiments 1 and 2, retrieval was more biased towards the category cluster 211 center for typical images relative to atypical images in the experimental group, $t_{(114)}=$ $2124.96, p<.001, d=.46$, but not the control group, $t_{(114)}=0.33, p=.74, d=.03$.

214 Error by category typicality: We also aimed to replicate the observed effects from 215 Experiments 1 and 2 showing more error for typical category members relative to 216 atypical category members (Figure S1). An analogous 2 (group: experimental, control) $\mathrm{x}$ 2172 (typicality: typical, atypical) ANOVA revealed a main effect of typicality, $F_{(1,228)}=4.09$, $218 p=.04, \eta_{p}^{2}=0.02$, no main effect of group, $F_{(1,228)}=0.85, p=.36, \eta_{p}{ }^{2}=0.04$, and no 219 reliable interaction, $F_{(1,228)}=2.84, p=.09, \eta_{p}^{2}=0.01$. For comparison with Experiments 2201 and 2 , we ran post-hoc t-tests to compare error by category typicality and group. As 221 observed in Experiments 1 and 2, we found greater error for typical images relative to 222 atypical images in the experimental group, $t_{(114)}=2.31, p=.02, d=0.22$, but not in the 223 control group, $t_{(114)}=0.28, p=0.78, d=0.03$. 


\section{Supplemental Methods}

Amazon Mechanical Turk: All data was collected on Amazon Mechanical Turk (AMT). AMT participants more closely match the demographics of adults in the United States relative to traditional lab experiments (Buhrmester, Kwang, \& Gosling, 2011) and the quality of data is equivalent to that collected from lab participants across a number of learning and memory experiments (Brady \& Alvarez, 2011; Crump, McDonnell, \& Gureckis, 2013). All experiments were restricted to participants who were located in the United States, had completed > 100 HITs with at least a 95\% acceptance rate, and had not participated in any prior pilots or other lab experiments that used the same stimuli.

234 These restrictions were chosen to increase the likelihood that participants were native 235 or fluent English speakers who could understand all provided instructions and were 236 naïve to the aim of the experiments. Depending on the length of the task, participants 237 were given a >5-hour time window to complete each experiment so they could choose a 238 suitable time to complete the tasks without interruption.

Software: Stimuli were presented using customized scripts written in HTML and JavaScript. MturkR was used to interface with AMT to post experiments, retrieve data, and pay participants (Leeper, 2017). All statistical analyses were conducted in base $\mathrm{R}$ 243 (R Core Team, 2015), with the exception of mixed-effects models, which were 244 computed using Ime4 (Bates, Mächler, Bolker, \& Walker, 2015). ImerTest was used to 245 provide statistics of main effects and interactions for mixed-effects models (Kuznetsova, 246 Brockhoff, \& Christensen, 2017), emmeans was used to compute estimated marginal 247 means to provide contrasts of simple their effects (Lenth, 2018), and bootES was used

248 to generate bootstrapped confidence intervals around their estimated effect sizes (Kirby 249 \& Gerlanc, 2013). All figures were generated with ggplot2 (Wickham, 2009).

\section{Experiment 1: Stimulus Development}

252 To create the image-location associations used in Experiment 1, we developed a data253 driven approach to estimate how images would be spatially organized if their locations 254 adhered to their category membership. To do this, we used relatedness ratings from a 
255 separate set of participants. These ratings were used both to define images' locations 256 and identify their category membership and typicality.

\section{Participants}

25924 participants ( $23-49$ years old, 9 female) completed the experiment. The University of Pennsylvania Institutional Review Board (IRB) approved all consent procedures.

\section{Materials}

Images: Stimuli consisted of $70150 \times 150$ pixel color images on white backgrounds (35 animals, 35 common objects) from the Bank of Standardized Stimuli (Brodeur, DionneDostie, Montreuil, \& Lepage, 2010; https://sites.google.com/site/bosstimuli/home). These images were re-sized to $100 \times 100$ pixels for the memory experiments.

\section{Procedure}

Picture naming: This task was developed to (1) ensure that participants could correctly identify the animals and objects, (2) familiarize the participants to the range of stimuli

271 over which they would be making similarity judgments, and (3) create a pool of qualified

272 participants that would be invited to complete the similarity ratings task. In this task,

273 participants were instructed to name each image as specifically as possible. For

274 example, if they viewed a picture of a bird, they were instructed to name the species of

275 bird if possible. On each trial, participants viewed an image and chose from three 276 options presented below the image: "I know this", "I know this but can't think of its 277 name", or "I don't know what this is". If they chose "I know this", they also typed the 278 name of the animal or object next to their choice. Either pressing the 'enter' key after 279 filling in the name, or clicking on the button corresponding to the other two choices, 280 automatically advanced the participant to the next trial with a $200 \mathrm{~ms}$ inter-trial-interval 281 (ITI). Otherwise, the trial advanced automatically after 15 seconds of inactivity. Trials 282 were divided into separate animal and object blocks that were randomized across 283 participants. The order of images in each block was randomized for each participant. On 284 average, the task was completed in 2 - 4 minutes. 
40 participants were recruited to participate in the picture-naming task. They were compensated with $\$ 1.50$. One participant was excluded for entering nonsense words for the majority of animal trials, and an extra participant was recruited as a replacement. As the aim of this task was to ensure that participants had sufficient knowledge of the animals and objects represented in each image, which participants may have even if they do not know the specific name of an image, we developed a lenient criterion for analyzing this task. Close neighbors of an image, such as crocodile

292 for alligator, lizard for gecko, utility knife or razor for box cutter, were accepted as correct. Misspelled names were included if they matched the correct image name with a maximum generalized Levenstein edit distance of 0.3 . Using these criteria, $94.93 \%$ of images (SD: 8.94\%) were named correctly. When including "I know this but can't think of its name" as accurate responses, accuracy rose to $97.71 \%$ (SD: $5.47 \%$ ), signifying near-perfect knowledge of the images. 35 participants who accurately named at least 65 out of 70 images were invited to participate in the next phase of the experiment; the excluded 5 participants named 39 - 62 images correctly.

Odd-man-out: Eligible participants were invited to participate in the odd-man-out (OMO) task. In this task, participants were presented with three animals or three objects and were instructed to click on the picture that 'did not belong', or in other words, the animal or object that was least similar to the other two. Once an image was chosen, the three images were replaced by three new images after a $200 \mathrm{~ms}$ ITI. Trials were untimed, but participants were instructed to respond in $2-4$ seconds and make their decision as quickly as possible while still being accurate. They were told that there were no right answers, but that some trials would seem easier than others. They were instructed to make their decisions based on many factors, instead of focusing on one. For animal trials, this included factors like whether they were in the same family, shared similar 311 habitats, and are predators or prey. For object trials, this included factors like whether they served a similar purpose, where they were commonly located, and their size.

For each category, a complete testing of all triplets of images would require 3146,545 trials (choose 3 given 35). Piloting of this task with a prior cohort $(\mathrm{N}=6)$ revealed 315 that for each participant, similarity matrices derived from a randomly sub-sampled set of 
316 two-fifths of these combinations were highly correlated with similarity matrices derived 317 from the participant's full set of trials (for all 6 pilot participants, animals: all $r>0.90$; 318 objects: all $r>0.81$ ). Thus, we presented participants with a random sample of 2,620 319 combinations per category (two-fifths of all possible triplets). This task was divided into 32020 separate batches (10 per category) expected to take 12 - 15 minutes each. Each 321 batch comprised 262 trials of a given category and 5 attention checks. In an animal 322 batch, an attention check comprised two animals and one object. Participants were 323 instructed to choose the object. In an object batch, participants were instructed to 324 choose the animal presented with two objects.

325 Although it is impossible to control the order of HITs that AMT workers choose to 326 work on, we attempted to randomize the order of batches that workers had access to by 327 creating six different sequences of batches, and assigning a worker to one of the 328 sequences based on the last letter or number in their account ID. AMT IDs are randomly 329 generated string of letters and digits, so assigning groups by the last letter or number of 330 a worker's ID is akin to sampling from a uniform distribution of 1 to 36 (26 letters, 10 331 digits). Within each batch, the trial order was randomized for each participant.

Participants were given 7 days to complete all 20 batches. Upon the invitation to 333 complete the batches, participants were told that they would be compensated $\$ 2.50$ per 334 batch, and if they completed all 20 within 7 days, they would receive a bonus of $\$ 5$, for a 335 total of $\$ 55$. Two reminders were sent over the course of the week, and a third reminder 336 was sent the day before the deadline, only to participants who had already completed $337>15$ batches. If a participant missed 2 or more attention checks in a batch, it was 338 rejected and a message was sent to the participant offering them an opportunity to re339 do the batch. Out of the participants whose data were used in the memory task, only 340 one participant needed to re-do a rejected batch. At the end of the week, 24 out of 35 341 participants had completed all 20 batches. The remaining 11 participants had completed 3421 to 13 batches (mean $=4, S D=4.44$ ).

343 The OMO judgments were then used to create pairwise similarity matrices for 344 each subject and super-ordinate category (Figures S6A, S6D). Starting with a $35 \times 35$ 345 matrix of zeros, for each trial in which an image was chosen, the similarity value for the 346 other two presented images increased by one. These similarity values were summed 
347 across all trials and then divided by the number of times the two images appeared in the 348 same trial. Similarity matrices thus ranged from 0 to 1 , with higher values corresponding 349 to greater similarity between images. To quantify the test-retest reliability of the ratings 350 for each participant, split-half correlations were derived by re-computing pairwise 351 similarity using the first and last half of the batches that were completed for each 352 subject, and then correlating the lower triangle of the two matrices together. 353 Correlations between the first and last half of batches were high (animals: mean $r=$ $3540.66, \mathrm{SD}=0.05$; objects: mean $r=0.54, \mathrm{SD}=0.06$ ) and greater than correlations 355 between each participant's ratings and similarity matrices derived from Latent Semantic 356 Analysis (LSA) and Word2Vec (Figures S6C, S6F). Three subjects, whose split-half 357 correlations for both categories were $>2$ SD lower than the group mean, were excluded 358 (all $r$ 's $<0.08$ ), leaving 21 subjects with data to be used to derive the locations for the 359 memory test.

Category membership and typicality: The odd-man-out results were used to derive a 2dimensional representation of participants' semantic knowledge (Figures S6B, S6E). To do this, the similarity matrices from the 21 participants who completed the odd-man-out task were averaged into two group-level similarity matrices for each super-ordinate category. Classical multi-dimensional scaling was used to project the matrices into $2 \mathrm{D}$ spaces. To evaluate how well this projection reflected participants' decisions in the OMO task, we computed the goodness of fit of the projected data to the full similarity space using subsets of the dimensions produced by the scaling procedure. This measure was operationalized as the $R^{2}$ between the full similarity matrix and a reconstructed matrix using 1 to 10 coordinate dimensions weighted by their eigenvalues

371 (Figure S6G). While there were no obvious bends in this elbow plot, moving from one 372 to two dimensions resulted in the steepest change in fit, and two dimensions captured $37385.5 \%$ of the variance in ratings for animals and $70.0 \%$ of the variance for objects. K-means clustering was applied to the $2 \mathrm{D}$ coordinates to derive data-driven 375 clusters of images. The optimal number of clusters was determined by computing the 376 within-cluster sum of squared error for solutions with 1 to 10 clusters and using an 377 elbow plot to identify the smallest number of clusters that explained the largest portion 
378 of the error (Figure $\mathrm{S} 6 \mathrm{H}$ ). For both categories, the data was best explained with 3 379 clusters. This data-driven procedure resulted in animal clusters corresponding to birds, 380 land mammals, and sea creatures, and object clusters corresponding to kitchen utensils 381 and appliances, tools and personal care items, and office supplies.

382 The cluster solutions were also used to identify images that were typical and 383 atypical category members. First, a category was operationalized as images belonging 384 to the same cluster (e.g. birds). The center of each cluster was derived by computing 385 the mean $x$ and $y$ coordinate over all cluster images. Then, all images were sorted by 386 their distance to their cluster center. For each cluster, the closest $20 \%$ of images were 387 labeled as 'typical' category members (e.g. cardinal), and the furthest $20 \%$ of images 388 were labeled as 'atypical' members (e.g. toucan and ostrich). Note that this data-driven procedure resulted in differing numbers of category members in each group, and thus 390 differing numbers of typical and atypical category members. Table 1 lists the number of 391 images per category and condition.

Counterbalancing: For some categories, the number of category members did not 394 evenly divide into $40 \%$, such that there were an odd number of images assigned as 395 spatially inconsistent that could not be evenly assigned to typical and atypical category 396 members. To account for this and for other variations in the stimulus display, we created 3976 counterbalancing groups. In each group, we (1) randomized the assignment of the 398 odd image to a typical or atypical category member, (2) generated a new, random set of 399 spatially inconsistent locations, and (3) counterbalanced whether animals appeared on 400 the left side of the screen and objects on the right, or vice versa. AMT workers were 401 assigned to one of the six groups based on the last letter or number in their Worker ID. 402 Worker IDs are randomly generated string of letters and digits, so using the last letter or 403 number of a worker's ID is akin to sampling from a uniform distribution of 1 to 36 (26 404 letters, 10 digits). 
A

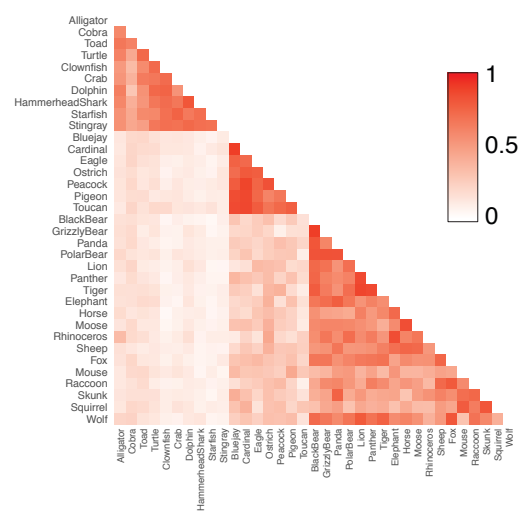

D

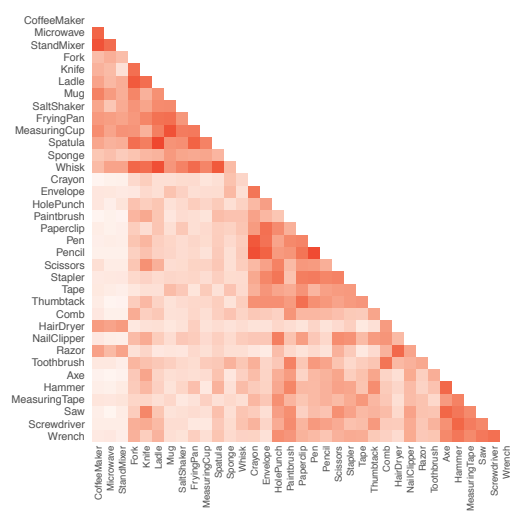

G
B

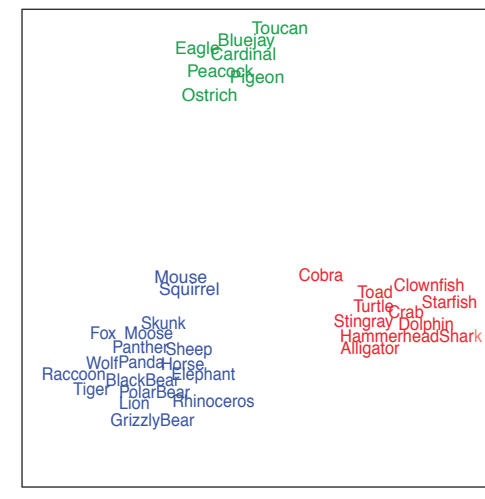

E

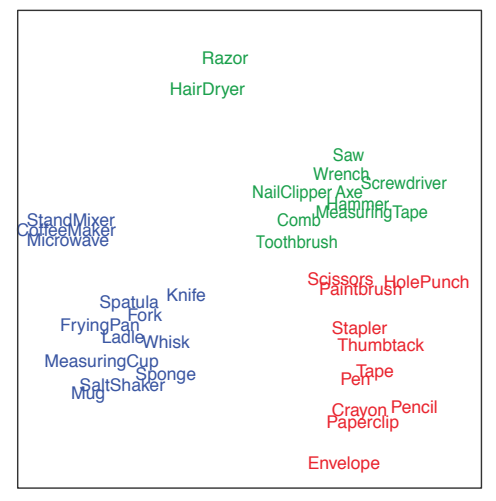

H
C

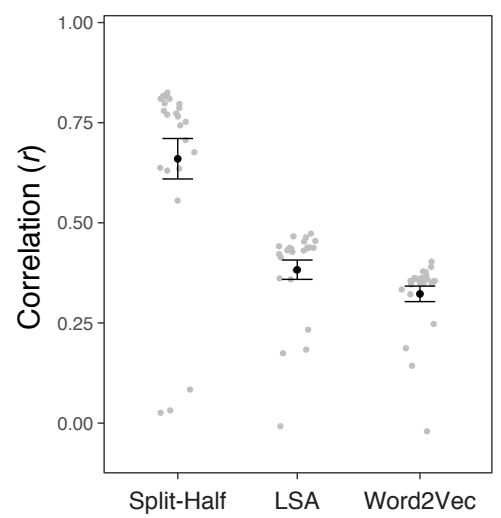

F

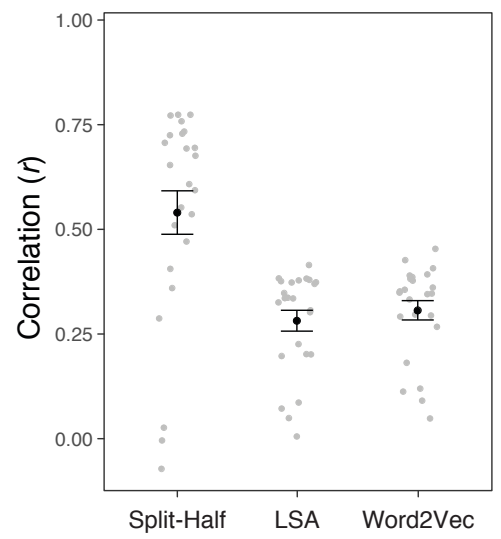

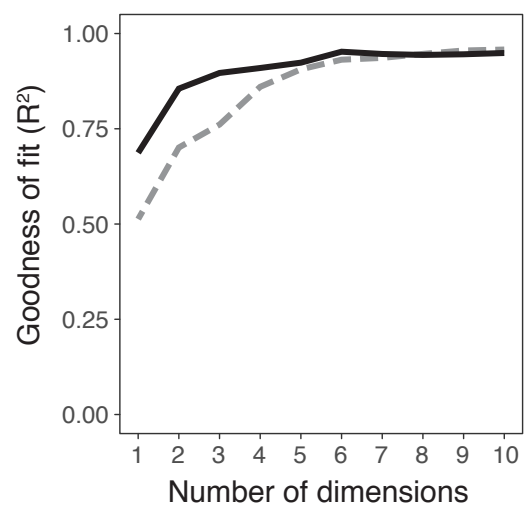

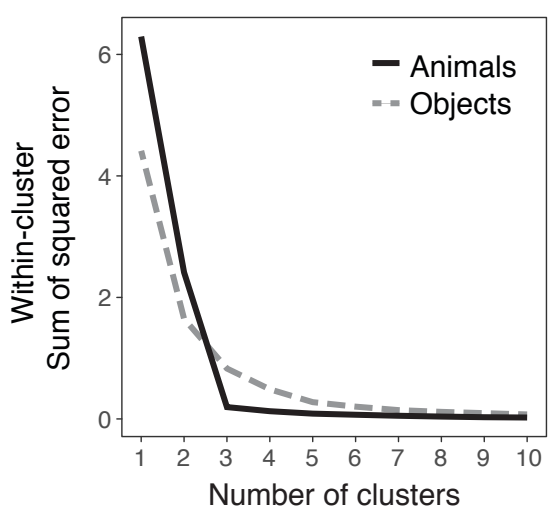

Figure S6. Odd-man-out procedure. (A, D) Similarity matrix of all animals and all objects matrices using classical multi-dimensional scaling. Colors indicates 3 clusters derived with kmeans clustering. (C, F) Split-half correlations of the similarity matrices within each participant plotted alongside each participant's correlations with similarity matrices derived from LSA and Word2Vec. Gray dots signify participants. Error bars indicate SEM. (G) Goodness of fit of projected similarity ratings derived from the group-averaged correlation matrix, as a function of 415 the number of reconstructed dimensions. (H) Elbow plot of within-cluster sum of squared error for the k-means clustering algorithm as a function of number of clusters chosen. 


\begin{tabular}{lllll}
\hline Experiments 1 \& 3 & & & & \\
\hline Category & Total & $\begin{array}{l}\text { Spatially } \\
\text { Consistent }\end{array}$ & Typical & Atypical \\
\hline Birds & & 4 & 1 or 2 & 1 or 2 \\
Mammals & 7 & 11 & 3 or 4 & 3 or 4 \\
Sea Creatures & 18 & 6 & 2 & 2 \\
Tools/personal care & 11 & 7 & 2 & 2 \\
Kitchen & 13 & 7 & 3 & 3 \\
Office & 11 & 7 & 2 & 2 \\
\hline Experiment 2 & & & & \\
\hline & Total & Spatially & Typical & Atypical \\
Category & & Consistent & & 3 \\
\hline Birds & 20 & 14 & 3 & 3 \\
Mammals & 20 & 14 & 3 & 3 \\
Sea Creatures & 20 & 14 & 3 & 3 \\
Insects & 20 & 14 & 3 & 3 \\
Clothes & 20 & 14 & 3 & 3 \\
Furniture & 20 & 14 & 3 & 3 \\
Kitchen & 20 & 14 & 3 & 3 \\
Office & 20 & 14 & 3 & \\
\hline
\end{tabular}

416

417

418

419

420

421

422

423

424

425

426

427

428

Table S1. Number of images in each category in each experiment. Top. Experiments $1,3 \& 4$. $40 \%$ of items in a category (the top and bottom $20 \%$ ) were assigned as spatially inconsistent. When this proportion gave rise to an odd number of items (e.g. in the bird and mammals category), the odd one was randomly assigned as typical or atypical for each counterbalancing group. In other words, a given counterbalancing group encoded one typical bird and two atypical birds, and another group encoded two typical birds and one atypical bird. Experiment 3 used the same categories, locations, and counterbalancing groups developed for Experiment 1. In Experiment 4, the number of items in each color category was randomly matched to a semantic category such that the number of items in each category varied in the same was as in Experiments 1 and 3. Bottom. Experiment 2. As categories were predetermined in Experiment 2, all categories comprised 20 items. $30 \%$ of items in a category (the top and bottom $15 \%$ ) were assigned as spatially inconsistent. 
430 We aimed to replicate Experiment 1 with validated approaches for defining category 431 membership and typicality. To this end, a separate cohort of participants completed a 432 list ranking task to identify typical and atypical members in a fixed set of categories.

\section{Methods}

\section{Participants}

436216 participants (27 per category) completed this task. The University of Pennsylvania 437 IRB approved all consent procedures. Demographics were not collected due to 438 experimenter error.

\section{Materials}

441 Stimuli comprised 160 100x100-pixel color images on white backgrounds. These 442 comprised 8 categories of 20 images: birds, insects, sea creatures, mammals, clothes, 443 furniture, kitchen utensils, and office supplies. The categories were selected from prior 444 studies of categorization norms (Uyeda \& Mandler, 1980; Deyne et al., 2008).

\section{Procedure}

447 We employed a validated list ranking task (Figure S7A; Djalal, Ameel, \& Storms, 2016). 448 Extensive instructions with examples were given to ensure participants understood the 449 concept of category typicality. For each category, participants viewed 20 images in a 450 box labeled 'Sort these'. Underneath, there were two empty boxes labeled 'Typical' and 451 'Atypical'. Participants were instructed to drag 10 images into each box. They were 452 allowed to drag images freely across the boxes in any order. Then, within each box, 453 participants sorted the 10 images as most (a)typical to less (a)typical. Arrows and labels 454 indicated the direction that images were to be sorted. The average completion time was 4552.6 minutes $(S D=1.9)$. The resulting positions of the images were concatenated into a 456 ranked list of category typicality and averaged across participants. The top three and 457 bottom three images in each list were assigned as 'typical' and 'atypical' category 458 members that would be 'spatially inconsistent' in the memory experiment, and the 459 images in the middle of the list were assigned as 'spatially consistent' (Figure S7B). 
A
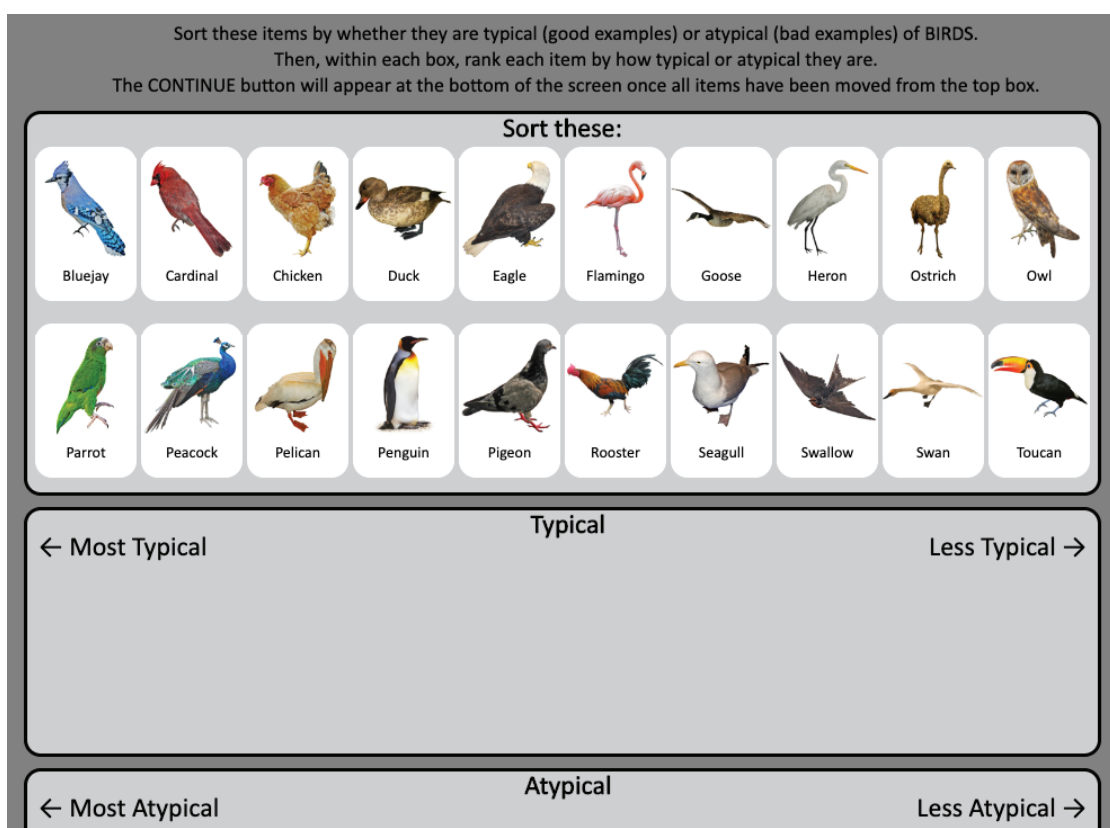

B
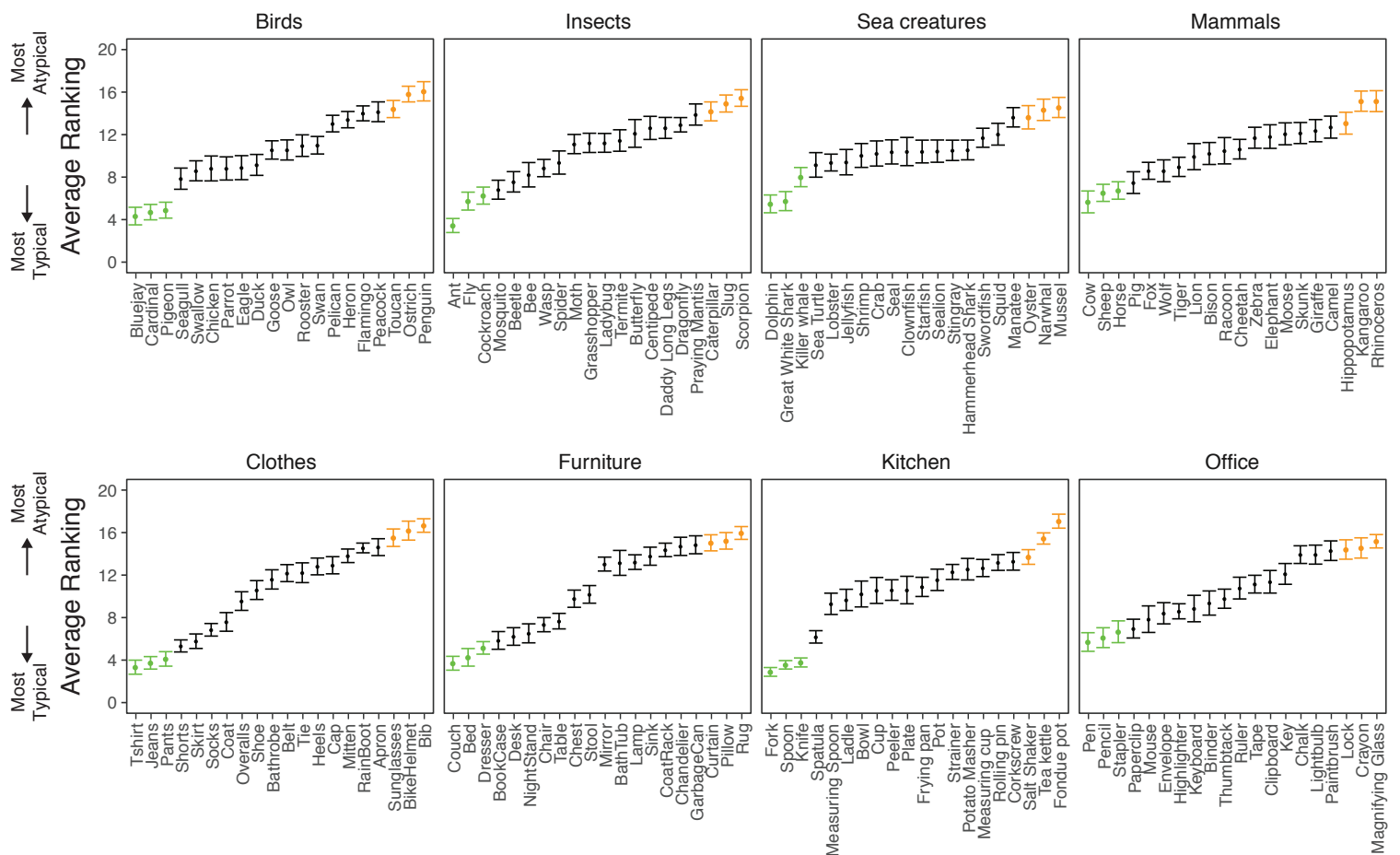

Figure S7. Ranking procedure (A) Ranking task and instructions for the bird category. (B) Average typicality rankings for each category, which could range from 1 (all participants ranked image as most typical) to 20 (all participants ranked image as most atypical). Error bars indicate SEM. Color indicates condition in the memory experiment: green = spatially inconsistent/typical; black = spatially consistent; orange $=$ spatially inconsistent/atypical. 
467 Power analysis: We chose a new sample size for this experiment to account for the fact 468 that the planned analyses were less powered relative to the analyses in the first two 469 experiments. Specifically, because we planned to analyze error and bias as a function 470 of exemplar memory accuracy, requiring the division of each condition by whether the 471 encoded exemplars were correctly or incorrectly chosen, there would be fewer trials in 472 each condition. To address this, we first piloted a subset of the shuffled control group ( $N$ $473=35)$ to procure an estimate of exemplar memory accuracy in the absence of 474 informative category knowledge during encoding. We found that accuracy was on 475 average $67.6 \%$ (SD 16.6\%). We then re-analyzed the influence of category typicality on 476 bias from the experimental group in Experiment $1(\mathrm{~N}=35)$ with a subset of their trials 477 that matched the number of exemplar incorrect trials from the pilot group. We chose to 478 conduct this re-analysis using incorrect trial counts because exemplar accuracy in the 479 initial subset was above 50\%, meaning there were fewer incorrect trials than correct trials and any analyses including only incorrect trials would be the least powered.

The subsampling procedure was as follows. First, we matched each participant in 482 Experiment 1 to a participant in the pilot cohort. We randomly subsampled the number 483 of typical and atypical images in the Experiment 1 participant to match accuracy from 484 the pilot participant and derived their average bias separately for typical and atypical 485 category members. We repeated this subsampling procedure 5,000 times per 486 participant. We then computed the effect size of the difference in bias for typical versus 487 atypical category members for each permutation and averaged them. This resulted in 488 an estimated effect size of the difference in bias by typicality that would have been 489 observed in Experiment 1 when limiting the analysis to trials with incorrect exemplar 490 memory, assuming both groups of participants exhibited equivalent memory for the 491 specific images.

492 As expected, this procedure resulted in a smaller effect size (Cohen's $d=0.263$ ) 493 than what was observed in Experiment 1 when including all trials (Cohen's $d=0.548$ ). 494 Using a power analysis $(\alpha=0.05$, power $=0.80)$, we established that a sample size of 495115 participants per group was needed to recover this smaller effect. 
497 Counterbalancing: In Experiment 4, we aimed match as many features of the 498 experimental design to Experiment 1 as possible in order to understand whether biases 499 in memory are driven by category knowledge or by newly-learned visual similarities of 500 encoded images. Because a data-driven clustering algorithm determined category 501 membership of the stimuli in Experiment 1, the number of category members varied 502 across categories (Table S1). Thus, to match this in Experiment 4, we created 6 503 counterbalancing groups, where in each one, the categories in Experiment 1 were 504 randomly matched to Experiment 4. For example, because in Experiment 1 there were 5057 birds, 18 mammals, and 10 sea creatures, in one counter-balancing group there were 5067 lamps, 18 clocks, and 10 chairs, and in another counter-balancing group there were 7 507 trains, 18 cars, and 10 planes.

508 As described in the main text, both the assignment of the six color ranges to the 509 six categories and the assignment of colors to images within each category were also 510 randomized separately for the 6 groups. Thus, each group encoded a unique set of 511 stimuli with (1) different numbers of category members per category, (2) categories with 512 different ranges of colors, and (3) different images assigned typical and atypical colors. 
514 Bates, D., Mächler, M., Bolker, B., \& Walker, S. (2015). Fitting linear mixed-effects models using Ime4. Journal of Statistical Software, 67(1), 1-48.

Brady, T. F., \& Alvarez, G. A. (2011). Hierarchical encoding in visual working memory: ensemble statistics bias memory for individual items. Psychological Science, 22(3), 384-392.

Brodeur, M. B., Dionne-Dostie, E., Montreuil, T., \& Lepage, M. (2010). The Bank of Standardized Stimuli (BOSS), a new set of 480 normative photos of objects to be used as visual stimuli in cognitive research. PLOS ONE, 5(5), e10773.

Buhrmester, M., Kwang, T., \& Gosling, S. D. (2011). Amazon's Mechanical Turk: A new source of inexpensive, yet high-quality, data? Perspectives on Psychological Science, 6(1), 3-5.

Crump, M. J. C., McDonnell, J. V., \& Gureckis, T. M. (2013). Evaluating Amazon's Mechanical Turk as a tool for experimental behavioral research. PLOS ONE, 8(3), e57410.

Deyne, S. D., Verheyen, S., Ameel, E., Vanpaemel, W., Dry, M. J., Voorspoels, W., \& Storms, G. (2008). Exemplar by feature applicability matrices and other Dutch normative data for semantic concepts. Behavior Research Methods, 40(4), 1030-1048.

Djalal, F. M., Ameel, E., \& Storms, G. (2016). The typicality ranking task: A new method to derive typicality judgments from children. PLOS ONE, 11(6), e0157936.

Kirby, K. N., \& Gerlanc, D. (2013). BootES: An R package for bootstrap confidence intervals on effect sizes. Behavior Research Methods, 45(4), 905-927.

Kuznetsova, A., Brockhoff, P. B., \& Christensen, R. H. B. (2017). ImerTest package: Tests in linear mixed effects models. Journal of Statistical Software, 82(1), 1-26.

Leeper, T. J. (2017). MTurkR: Access to Amazon Mechanical Turk Requester API via R.

539 Lenth, R. (2018). emmeans: Estimated Marginal Means, aka Least-Squares Means.

540 R Core Team. (2015). R: A Language and Environment for Statistical Computing.

$541 \quad$ Vienna, Austria: R Foundation for Statistical Computing.

542 Uyeda, K. M., \& Mandler, G. (1980). Prototypicality norms for 28 semantic categories.

543 Behavior Research Methods \& Instrumentation, 12(6), 587-595.

544 Wickham, H. (2009). ggplot2: Elegant Graphics for Data Analysis. Springer-Verlag: New 545 York. 